\title{
Claude Lagadec
}

(1996)

\section{Les Fondements biologiques de la morale Essai pour introduire la méthode expérimentale en morale}

Un document produit en version numérique conjointement par Jean-Marie Tremblay et Marcelle Bergeron, bénévoles

Courriels : jean-marie_tremblay@uqac.ca et mabergeron@videotron.ca

Dans le cadre de la collection : "Les classiques des sciences sociales" dirigée et fondée par Jean-Marie Tremblay, professeur de sociologie au Cégep de Chicoutimi Site web: http://classiques.uqac.ca/

Une collection développée en collaboration avec la Bibliothèque Paul-Émile-Boulet de l'Université du Québec à Chicoutimi Site web: http://classiques.uqac.ca 


\section{Politique d'utilisation de la bibliothèque des Classiques}

Toute reproduction et rediffusion de nos fichiers est interdite, même avec la mention de leur provenance, sans l'autorisation formelle, écrite, du fondateur des Classiques des sciences sociales, JeanMarie Tremblay, sociologue.

Les fichiers des Classiques des sciences sociales ne peuvent sans autorisation formelle:

- être hébergés (en fichier ou page web, en totalité ou en partie) sur un serveur autre que celui des Classiques.

- servir de base de travail à un autre fichier modifié ensuite par tout autre moyen (couleur, police, mise en page, extraits, support, etc...),

Les fichiers (.html, .doc, .pdf., .rtf, .jpg, .gif) disponibles sur le site Les Classiques des sciences sociales sont la propriété des Classiques des sciences sociales, un organisme à but non lucratif composé exclusivement de bénévoles.

Ils sont disponibles pour une utilisation intellectuelle et personnelle et, en aucun cas, commerciale. Toute utilisation à des fins commerciales des fichiers sur ce site est strictement interdite et toute rediffusion est également strictement interdite.

\section{L'accès à notre travail est libre et gratuit à tous les utilisateurs. C'est notre mission.}

Jean-Marie Tremblay, sociologue

Fondateur et Président-directeur général, LES CLASSIQUES DES SCIENCES SOCIALES. 
Cette édition électronique a été réalisée conjointement par Jean-Marie Tremblay, et Marcelle Bergeron, bénévoles, respectivement professeur de sociologie au Cégep de Chicoutimi, d'une part, et professeure retraitée de l'enseignement à la Polyvalente Dominique-Racine de Chicoutimi, à partir du livre de :

Claude LAGADEC

\section{Les Fondements biologiques de la morale. Essai pour introduire la méthode expérimentale en morale. Montréal : Les Éditions Les Herbes Rouges, 1996, 213 pp. Collection : Essais.}

Madame Hélène Lagadec, sœur de Claude Lagadec et ayant droit des œuvres de son frère, nous ont accordé le 26 mars 2008 son autorisation de diffuser la totalité des publications de son frère dans Les Classiques des sciences sociales.

\section{gf Courriel : hlagadec@hotmail.com}

Numérisation, reconnaissance de caractères et correction : Jean-Marie Tremblay;

Mise en page : Marcelle Bergeron.

Polices de caractères utilisés :

Pour le texte : Times New Roman, 12 points.

Pour les citations : Times New Roman 10 points.

Pour les notes de bas de page : Times New Roman, 10 points.

Édition électronique réalisée avec le traitement de textes Microsoft Word 2003 pour Macintosh.

Mise en page sur papier format : LETTRE (US letter), 8.5'’ x 11'’)

Édition numérique réalisée le 15 mai 2008 à Chicoutimi, Ville de Saguenay, province de Québec, Canada. 
Claude LAGADEC

(1996)

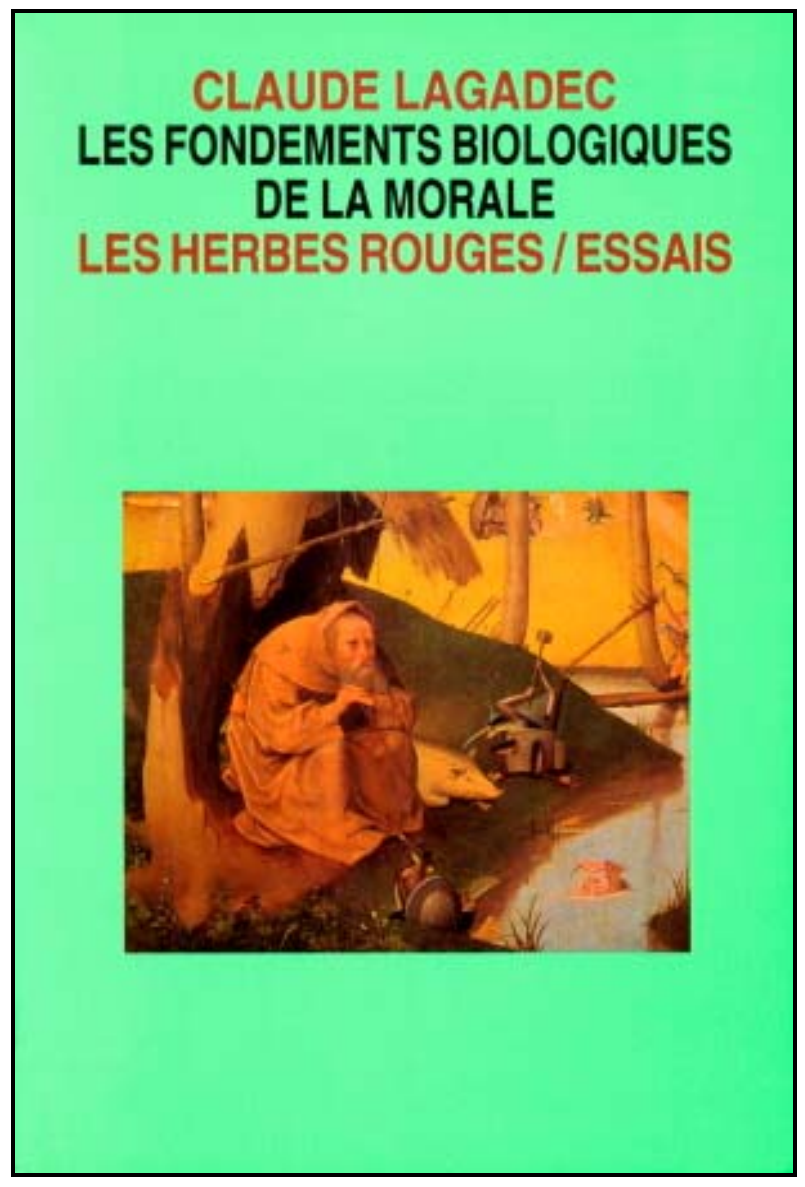




\section{Table des Matières}

$\underline{\text { Index }}$

$\underline{\text { Introduction }}$

Chapitre 1.

La morale et la liberté

Chapitre 2.

Plaidoyer pour une morale expérimentale

Chapitre 3.

Le grand oublié : le groupe

Chapitre 4.

La question du racisme

Chapitre 5.

L'improbable

Bibliographie 


\section{DU MÊME AUTEUR}

Dominances. Essai de sociobiologie de l'inégalité et de la tromperie, Éditions Le Préambule, 1982.

La Morale de la liberté, Éditions Le Préambule, 1984 
INDEX

$\underline{\text { Retour à la table des matières }}$

agressivité

altruisme

APOSTLE, Richard A.

ARISTOTE

AUGUSTIN (saint)

AUSTER, Paul

BACON, Francis

BATESON, Gregory

BENEDICT, Ruth

BERNARD, Claude

BOHR, Niels

BOSCH, Jérome

BOYD, Robert

BROWN Jr., William L.

CAMPBELL, D.T.

CAMUS, Albert

CASTANEDA, Carlos

COLLINS, Anne

compulsion contrainte

contrainte primaire

contrainte secondaire

contrat social

CORNFORD, F. M.

DARWIN, Charles

DAWKINS, Richard

DE GOUGES, Olympe

DELEUZE, Gilles

démocratie

DESCARTES, René

DOBZHANSKY, Theodosius

DODDS, E.R.

DOVIDIO, John F.

DURKHEIM, Émile

égalité
EINSTEIN, Albert

EKELAND, Ivar

ÉPICTÉTE

épidémiologie

ethnocentrisme

FLACELIÈRE, Robert

FORSÉ, Michel

FOUCAULT, Michel GALIEN,

Claude

GALILÉE

GAUSE, G. J.

GOETHE, Johan Wolfgang

GOULD, Stephen Jay

groupe

HAMILTON, William D

HARDIN, Garrett

HAVELOCK, Eric A.

HÉRACLITE

HILLMAN, James

HIPPOCRATE

HOBBES, Thomas

HOSKEN, Fran

HULSE, Frederick S.

HUME, David

IKE, Ben W.

ILLICH, Yvan

immoralité

improbable

inceste

Islam

jalousie

JUNG, Carl 
KANT, Immanuel

KEPLER, Johannes

KORZYBSKI, Alfred

LAGADEC, Claude

LAVOISIER, Antoine

LÉVI-STRAUSS, Claude

LEVINE, R.A.

LEWIN, Kurt

liberté

libre arbitre

LICHTENHAELER, Charles

LUMSDEN, Charles J.

MABBOTT, John David

MAGENDIE, François

MALTHUS, Robert Thomas

MARX, Karl

mauvaise herbe

MAYNARD

SMITH, John

MAYR, Ernst

McLUHAN, Marshall

médecine

milieu intérieur

MINEAR, Richard $\mathrm{H}$.

MOORE, George Edward

morale expérimentale

morale spontanée

musulman

négligence

NEWTON, Isaac

NIETZSCHE, Friedrich

non-vérité

PASCAL, Blaise

petit groupe

Phéniciens
PIRSIG, Robert M.

PLATON

positivisme

probable

PUTNAM, Hillary

racisme

RAWLS, John

RESCHER, Nicholas

REYNOLDS, V.

RIBOT, Théodule

RICHERSON, Peter J.

ROUSSEAU, Jean-Jacques

RUSE, Michael

RUSHDIE, Salman

RUSSELL, Bertrand

SARTRE, Jean-Paul

sexisme

SOUTHWICK, C.H.

STEINER, George

suicide

TAINE, Hippolyte

TIGER, Lionel

TODOROV, Zvetan

trahison

tribu

TWAIN, Mark

valeur

value-free

VAN DER DENNEN, M.G.

VERNANT, Jean-Pierre

VIDAL-NAQUET, Pierre

VON HUMBOLDT, Wilhem

WILSON, Edward O.

xénophobie 


\section{Quatrième de couverture}

Pourquoi les sciences sociales ne sont-elles pas devenues de véritables sciences comme elles l'avaient promis ? Si l'on reconnaît avec Nietzsche que les valeurs sont des contraintes imposées par le groupe il s'ensuit que chaque version de la morale humaine emporte avec elle sa forme propre d'immoralité. Les scientifiques sont aussi un groupe et c'est pourquoi la science n'est pas un objet de science.

Cet ouvrage propose la création d'une science de la morale, expérimentale et appliquée. La liberté c'est la vie elle-même, ce que la mécanique classique appelle l'entropie négative. La liberté c'est l'ordre que la vie oppose au désordre de la physique. La morale est l'ensemble des contraintes engendrées par cette liberté ; elle doit être interprétée dans le cadre de la théorie évolutionniste de Darwin. Le premier objet d'une science de la morale pourrait être l'étude du racisme. Si l'hypothèse avancée ici d'une xénophobie animale sociogène était démontrée par voie expérimentale, notre compréhension et nos moyens d'intervention contre les effets indésirables du racisme s'en trouveraient considérablement renouvelés.

Claude Lagadec est né à Montréal. Il a enseigné la philosophie à l'Université de Montréal, à l'Université McGill et à l'Université du Québec à Montréal. Il est l'autour de Dominances. Essai de sociobiologie de l'inégalité et de la tromperie et de La Morale de la liberté. 


\section{REMERCIEMENTS}

Je veux remercier en tout premier lieu le professeur Yvon Gauthier du département de philosophie de l'Université de Montréal, qui m'a fourni son aide au cours de nombreuses années en matière de physique et de logique. Je remercie également le professeur Pierre Gravel du même département pour le partage de ses connaissances en philosophie grecque. Je veux aussi témoigner ma reconnaissance à la mémoire du regretté Jean-Paul Audet qui m'a fait partager son savoir inestimable et sa sagesse, particulièrement sur les cultures et les sociétés préchrétiennes et du Moyen-Orient. Ma reconnaissance va également à PauleEsther Gagnon, Louise Mailloux et Georges Rousseau qui m'ont encouragé par leurs observations à divers stades de la rédaction du manuscrit. Yves Couture et Roger Tessier sont remerciés pour leur contribution. Merci au professeur Serge Robert du département de philosophie de l'Université du Québec à Montréal pour ses conseils judicieux et le grand soin apporté à l'étude du manuscrit. Merci enfin au professeur Michel Morin, directeur de la collection « Essais » et à ses collègues Claude Bertrand, Pierre Bertrand, François Raymond, et à Nicolas Bertrand. 


\section{Introduction}

$\underline{\text { Retour à la table des matières }}$

Pouvons-nous encore croire que les sciences sociales deviendront un jour moralement neutres comme elles ont promis de le devenir depuis le jour de leur naissance, value-free comme disent les Américains ? Telle était la question qui fut à l'origine de la présente recherche. Cette recherche a entraîné le réexamen des idées de valeur et d'immoralité et il semble que la réponse doive être plutôt négative.

C'est là un problème permanent du discours des sciences sociales, car alors que nous n'avons qu'une seule théorie de l'électricité, par exemple, il n'en va pas de même en sociologie, en anthropologie ou en psychologie sociale où l'on voit chacune de ces prétendues sciences varier selon la diversité des cultures qui les alimentent, du lieu de production et de la date de naissance de la théorie ayant cours. Alors qu'en électricité la loi d'Ohm, de 1827, $\mathrm{R}=\mathrm{V} / \mathrm{I}$ (la résistance est égale à la différence de potentiel divisée par l'intensité du courant) vaut en tous temps et en tous lieux, en sciences sociales il semble que ce soit différent. Il existe une science sociale dont l'aire de diffusion est limitée à la tradition anglaise alors qu'une autre est plus influencée par la tradition américaine, une autre encore est française, jusqu'à récemment l'une était marxiste et il y en a d'autres encore. Ce qui signifie autant de conceptions ou d'interprétations de ce que c'est que le pouvoir, la culture, le rôle et l'importance du langage, des artéfacts, etc. Ces diverses conceptions du social ne sont pas nécessairement incompatibles entre elles, mais leur diversité fait problème.

Cette situation existe depuis le milieu du siècle dernier, lorsque les sciences sociales furent crées par l'application des méthodes des sciences « dures », comme la physique ou la chimie, aux affaires humaines. Il semblerait donc que ce n'est pas par les méthodes qu'elles utilisent que les sciences sociales diffèrent des autres sciences mais plutôt par leur objet : les choses humaines. Pourquoi chaque société a-t-elle ainsi tendance à produire sa propre version de la sociologie, de l'anthropologie et des autres sciences sociales ? Si l'on admet que la morale humaine, telle que perçue par la conscience individuelle, subit l'influence de facteurs propres à chaque société, ce qui semble peu contestable, il est possible que la morale colore chaque version culturelle du savoir sociologique. La morale 
implicite du discours sociologique le destinerait ainsi à la consommation locale seulement.

Si l'on voulait « épurer » la science sociale de son contenu moralisateur actuel et de ses « valeurs » implicites, il faudrait savoir ce que l'on veut lui enlever et qui serait susceptible de la rendre inadéquate. Si l'objet à constituer est une science, il faudrait posséder une connaissance scientifique, ou au moins objective, de ce qu'est la « valeur ». Pour épurer un panier d'oranges du citron qui s'y trouverait par mégarde je dois savoir exactement ce que je cherche. Mais dans la cas de la morale personne à l'heure actuelle, y compris les philosophes, n'est en mesure de décrire en termes objectifs ce que sont au juste nos "valeurs ", de dire de quoi elles sont faites et ce que nous entendons par là, bref d'en donner une description non limitée aux frontières culturelles. Le terme de « valeur » est présentement... affecté d'une « valeur », si l'on ose dire, il est en quelque sorte auto-justificatif. C'est là le cœur du problème. On peut estimer que pour le moment le terme est non seulement peu éclairant mais véritablement trompeur et qu'il serait prudent de l'éviter dans le contexte d'un discours logique ou cohérent. Pourtant son utilisation est fréquente précisément dans les textes savants affirmant que la science est ou doit être valuefree, et dans le langage ordinaire qui l'exprime.

Il serait peu utile de nous contenter ici de faire une critique du langage ordinaire pour tenter de l'épurer. Il n'est pas évident que les sciences sociales font un usage vraiment inconsidéré du langage lorsqu'elles évoquent les « valeurs ». Le problème est beaucoup plus profond, c'est l'idée même de notre morale qui est enjeu et que le présent ouvrage tentera de reformuler. C'est une tâche de philosophe.

La suite de la présente introduction fera un bref examen des raisons qui nous font rejeter l'usage du terme « valeur » dans son acception actuelle. Une définition objective de ce que nous signifions par le terme de "valeur » sera proposée au chapitre 1 dans le cadre de définitions objectives de la morale et de la liberté humaines. Dans l'intervalle le terme de «valeur» sera constamment mis entre guillemets.

Le chapitre 2 proposera les principes généraux d'une science expérimentale de la morale dont la création est proposée. C'est le sens du sous-titre du présent ouvrage, inspiré du sous-titre du Traité de la nature humaine de David Hume publié en 1739 qui était : Essai pour introduire la méthode expérimentale dans les questions morales. Le chapitre 3 évoquera la difficulté et l'importance, aux fins d'une science de la morale, de l'idée de groupe. Ces considérations se prolongeront dans le chapitre 4 par l'examen de la question du racisme. Le chapitre 5 fera l'éloge de l'improbable. 
Je dois dire ici un mot des difficultés particulières rencontrées dans la rédaction de ce livre et qu'il peut être utile au lecteur de connaître. Après que le chapitre 1 et le chapitre 2 eurent été rédigés d'une seule traite je possédais déjà une bonne idée de ce que serait le chapitre 5 qui est le seul dans lequel je prends personnellement parti comme philosophe et comme moraliste ; je savais également dès ce moment que ce chapitre 5 serait d'inspiration nietzschéenne, ce qui est assurément inattendu dans un livre faisant largement appel à la biologie. Mais la grande surprise, qui demeure très vive encore à ce jour, est survenue dans la rédaction des chapitres 3 et 4 qui ont imposé à cet ouvrage une sorte de « développement par le milieu ». La surprise fut de devoir reconnaître, malgré tout ce que nos maîtres nous ont enseigné et malgré tout ce que je croyais savoir, que le fondement de la morale humaine se situe dans le groupe et non pas dans la personne individuelle ou dans sa rationalité. Le groupe étant une invention de la vie, de l'évolution, je dus alors reconnaître que les fondements de la morale sont essentiellement biologiques. Le chapitre 3 portant sur le groupe, de même que le chapitre 4 portant sur le racisme et la xénophobie, sont ainsi devenus l'avers et le revers du même et immense problème moral que demeure l'ethnocentrisme, problème sur lequel je reviendrai fréquemment en cours de rédaction et qui demeure à ce jour l'obstacle le plus important à une compréhension systématique de la morale humaine.

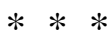

\section{Les « valeurs " en philosophie}

Le terme « valeur » est relativement récent dans l'histoire des idées en Occident. Il ne s'est guère répandu avant la seconde moitié du siècle dernier lorsque des néokantiens, surtout allemands, l'ont repris de Kant et développé. Il apparaît aussi dans la Philosophie de l'art de Taine en 1892, et chez Ribot (1839-1923), l'un des fondateurs de la psychologie expérimentale en France. L'idée, sinon le terme, est omniprésente dans l'œuvre de Nietzsche. À l'heure actuelle, mais surtout depuis la seconde moitié de notre siècle, non seulement l'idée de « valeur » a peu à peu cessé d'être l'objet de développements théoriques, mais aussi l'ensemble du discours moral fonde sur elle a souffert de cette indigence. " Nous vivons, disait Einstein, en un temps où les moyens sont d'une grande perfection, et les buts d'une grande confusion. » Ce qui résume assez bien l'espèce de désert où se trouve présentement la réflexion sur les « valeurs ». On avait pu croire il n'y a pas si longtemps encore, quelques dizaines d'années à peine, que la critique des idéologies produite par les sociologues remplacerait avantageusement le discours sur les "valeurs » et même le discours moral lui-même. Puis les années passèrent, la critique des idéologies semble passée de mode ; pourtant nous sommes toujours des êtres moraux et nous continuons à parler de nos « valeurs ».

Pour des raisons qui deviendront évidentes dans le chapitre 1, on ne trouvera pas ici d'examen exhaustif des diverses significations attachées à l'idée de

« valeur » dans les textes devenus classiques. Il convient cependant de situer la 
question en procédant à un bref examen de ce que l'on entend d'ordinaire par ce terme. À cette fin, il suffira d'examiner l'idée d'égalité et du rôle qu'elle joue en tant que « valeur».

Au deuxième paragraphe de la Déclaration américaine d'indépendance de 1776, on peut lire: "Nous tenons ces vérités pour évidentes par elles-mêmes, que tous les hommes sont créés égaux. » L'idée d'égalité, aussi présente dans la Déclaration des droits de l'homme et du citoyen en France en 1789 et dans ses versions subséquentes, est certainement l'une de nos « valeurs ».

La première question que nous pouvons nous poser ici concerne l'absence de perspective historique dans l'usage que nous faisons du concept d'égalité en tant que « valeur ». Si l'égalité était une « valeur » en 1776, pourquoi ne l'était-elle pas un ou deux siècles auparavant sous l'Ancien Régime ? Sachant que ce qui apparaît un jour peut disparaître un jour, on voudra savoir de quel matériau est fait ce que nous appelons une « valeur », d'où elle provient et ce qui la constitue. Qu'est-ce, au juste, qu'une « valeur »?

Le deuxième problème découle du premier et il est plus important. Le contenu pratique de ce que nous appelons, aujourd'hui, nos «valeurs », peut être très différent du contenu historique qu'avaient les mêmes "valeurs » telles que l'entendaient nos devanciers dont nous continuons cependant à nous réclamer comme faisant autorité. Le mot égalité est le même, en 1776, que celui que nous utilisons de nos jours, mais ni les Américains ni les Français révolutionnaires ne songèrent, à leur époque, à inclure les femmes dans ce qu'ils appelaient l'égalité des hommes : le terme homme n'était pas générique. C'est gênant, les femmes étant la moitié de l'humanité. Olympe de Gouges, en France, a vainement tenté de faire accepter par les révolutionnaires une Déclaration des droits de la femme et de la citoyenne. Les Américaines n'ont finalement obtenu le droit de vote qu'en 1920, les Françaises en 1944, et la Constitution américaine ne contient pas encore, à ce jour, l'amendement réclamé par les féministes qui reconnaîtrait explicitement l'égalité de l'homme et de la femme.

Un problème analogue se pose au sujet des Noirs américains. Les signataires de la Déclaration américaine, dont plusieurs étaient propriétaires d'esclaves, n'incluaient pas le Noir dans le terme homme. Les États-Unis n'ont aboli l'esclavage qu'un siècle plus tard, en 1865 ; il fallut attendre que passe encore un autre siècle, jusqu'en 1959, avant que les Noirs américains ne s'engagent finalement dans la grande lutte des Civil Rights. À ce jour leur lutte est loin d'être terminée. Qu'est-ce donc que la « valeur » égalité ?

Une «valeur » est certes un point de rencontre des esprits, un point de ralliement dans l'histoire des idées et des nations. Mais que se passe-t-il vraiment et que doit-on penser de ce genre d'évolution historique qui nous fait utiliser le même terme que les hommes de 1776 et 1789, mais dans un sens si différent du 
leur qu'eux-mêmes l'auraient certainement répudié si l'on avait en leur temps sollicité leur avis ? Pour nous-mêmes, le sens que nous donnons à ce terme est si différent que le leur nous apparaît aujourd'hui insupportable, et néanmoins nous continuons à nous réclamer d'eux. Ne devrions-nous pas, nous mêmes, nous dissocier d'avance de ceux de nos descendants qui, dans l'avenir, pourraient être tentés de se réclamer de leurs devanciers, nous-mêmes, avec la même légèreté que nous adoptons avec les nôtres?

Car s'il est vrai que, de notre point de vue, le fait d'inclure la femme et le Noir dans l'idée d'égalité comme "valeur » l'améliore et l'enrichit à nos yeux, nous l'avons aussi appauvrie pour des yeux d'hommes d'autrefois. Par exemple, nous avons perdu le sens de l'honneur de l'homme du XVIII ${ }^{\mathrm{e}}$ siècle, cette très haute estime de soi et de sa communauté, qui pouvait l'obliger à risquer sa vie dans un duel. Même au cours de la guerre de 1914-1918, un officier devenu prisonnier pouvait encore donner à ses geôliers ennemis sa parole d'honneur de ne pas tenter de s'évader, et s'y tenir ; le non-respect de la parole donnée eût constitué une déchéance, la perte du sens même de l'honneur. Personne n'a demandé aux officiers américains faits prisonniers au Vietnam et qui avaient trahi des secrets militaires pourquoi ils ne se sont pas suicidés.

Je ne veux pas laisser entendre que notre notion actuelle de l'égalité comme « valeur » est fautive ou que nous avons tort de lui conférer ce statut et de lui faire jouer ce rôle, mais seulement faire remarquer combien ce statut et ce rôle sont bizarres et difficiles à situer parmi nos autres connaissances. Nous pensons notre morale en termes de « valeurs », telle l'égalité ; nous savons que ces « valeurs » ne sont pas totalement sans précédents mais l'idée que nous avons de leur histoire est fortement sélective, c'est-à-dire plus ou moins arbitraire. Nous ne nous référons à des conceptions passées de nos valeurs actuelles que dans la mesure où les anciennes semblent conforter l'autorité des nôtres, laissant tomber le reste dans une sorte de néant que nous préférerions oublier, comme le souvenir d'un grand-oncle qui aurait été de mauvaise vie. Ou chimpanzé.

\section{Nos « valeurs » et celles des autres}

Ces considérations portaient sur la «valeur» selon le temps, On peut aussi comparer selon l'espace, c'est-à-dire examiner les « valeurs » propres aux sociétés autres que la nôtre. Les énoncés de nos traités de morale, déclarations de principes, comme les chartes des droits de la personne, visent généralement l'humanité tout entière. Et cependant, la morale humaine, qui se dit universelle, est toujours particulière à un groupe et en pratique peu exportable.

Cette caractéristique de la morale humaine est connue depuis toujours, je crois bien. "Plaisante justice qu'une rivière borne!", s'exclamait Pascal dans ses Pensées. "Vérité au deçà des Pyrénées, erreur au delà. » Mais s'il moque les fantaisies et les caprices des Allemands et des Perses, ce n'est que pour mieux 
persuader son lecteur d'embrasser sa morale à lui et la croyance en Jésus-Christ. Sur ce point, et en dépit de l'extraordinaire richesse de nos connaissances alimentées par les témoignages des anthropologues sur la diversité des mœurs des peuples de la terre, notre connaissance de la morale n'a pas avancé d'un pas. Notre morale, qui continue à se présenter comme universelle, continue non seulement à être une morale parmi d'autres, particulière et donc non universelle, mais elle continue aussi à déprécier toutes les autres qui ne lui ressemblent pas.

Dois-je vraiment donner des exemples? Je me contenterai d'un seul. En Occident de tradition chrétienne, le mariage est libre et monogame : un homme choisit librement, c'est-à-dire sans pressions extérieures, d'épouser une seule femme, celle-ci, qui de son côté choisit librement d'épouser un seul homme, celuilà. Par ailleurs, en Occident, l'alcool est moralement permis, il est même sanctifié dans le principal rituel de l'Église catholique. En Islam, par contre, le mariage est polygame (un homme peut épouser quatre femmes) ; et il n'est certainement pas libre au sens occidental et ne dépend pas de la seule volonté des époux mais implique l'intervention effective de chacune de leurs communautés respectives. Et en Islam, par ailleurs, l'alcool est immoral par lui-même. Le Mépris ou la répugnance de l'Occidental pour le mariage musulman n'a d'égal que la conviction musulmane que les mœurs occidentales sont dégénérées, particulièrement dans ces débits d'alcool où l'on voit des femmes, dont ce n'est pas la place, servir les clients ou consommer elles-mêmes.

Lorsque Bertrand Russell a écrit son histoire de la philosophie, il l'a intitulée : Histoire de la philosophie occidentale, reconnaissant ainsi le caractère limité de son entreprise et du corpus où il puisait. Je ne connais pas de traité de morale dont le titre dise expressément sa particularité d'être occidentale, et donc partielle. Par défaut, logiquement, l'absence de quantificateur confère à cette particularité la prétention à l'universalité. Prenons, par exemple, l'ouvrage de G.E. Moore, l'ami de Russell, publié en 1903 : Principia Ethica. Le philosophe moraliste n'aurait-il pas dû signaler dans son titre que son traité était réservé à l'usage des Anglais ? Ou des anglophones ? Ou des Occidentaux?

Ici encore, je ne tiens pas, pour le moment, à tenter de corriger ce langage mais plutôt à le comprendre, à comprendre comment il opère. Car la particularité effective du discours moral qui se donne pour universel est un phénomène beaucoup trop généralisé pour qu'une simple modification langagière puisse faire autre chose que maquiller le problème et le rendre encore un peu plus incompréhensible par cette opération cosmétique. Il se trouve en effet que le moraliste G.E. Moore appartient à une tradition philosophique qui a fait de la correction du langage l'un de ses principaux idéals. N'est-ce pas un véritable tour de force que de réussir ainsi à toujours présenter le particulier sous la forme de l'universel, surtout pour un spécialiste du langage et surtout en morale ? Pour s'en convaincre, il suffirait de traduire Principia Ethica et le mettre à la portée de lecteurs asiatiques ou arabes pour que la même correction langagière, mais dans 
une autre langue, produise des « effets de lecture » et des réponses très différentes à la très anglaise question : What is Good ?

Ce que je veux savoir, c'est comment cette morale fonctionne, comment nous nous y prenons pour que, même en toute correction langagière, la particularité de la conviction morale se véhicule uniformément sous le mode d'une apparente universalité. Car ce phénomène semble sans exception qui fait que toute société, primitive ou industrielle, en langage ordinaire ou au contraire savant, présente sa morale particulière comme la plus sublime réalisation de l'humanité. Même si nous parvenions à nous moquer de l'étroitesse d'esprit ou du prosélytisme de Pascal, notre conception de la morale qui n'a guère progressé sur ce point reproduirait encore la même aberration. Y compris lorsque, à la question de savoir ce que c'est que le Bien, nous sommes tentés de répondre que ça dépend de l'origine de la personne qui interroge et de celle qui répond. Car même le prétendu « relativisme moral » dans lequel le locuteur pourrait se réfugier verbalement n'est précisément que cela : une autre astuce langagière sans conséquence, facile à dire, impossible à appliquer dans la pratique. Il ne semble pas en notre pouvoir d'outrepasser le particularisme moral ni de l'empêcher de se présenter sous la forme d'une prétention à l'universalité.

Il ne faut pas dire, non plus, que ce problème est insoluble. Matériellement, je veux dire dans les faits, la résolution de ce problème existe bel et bien, elle est même d'une indépassable économie de moyens et d'une simplicité enfantine : il suffit que personne n'en parle et que personne ne dise que le roi est tout nu. Il suffit que chaque moraliste persévère, sérieux, bien intentionné et compétent et qu'il continue ainsi à publier chacun à tour de rôle ses propres Principia Ethica sans faire la moindre allusion à l'existence d'autres morales et d'autres discours tout aussi sérieux, bien intentionnés et compétents que le sien mais incompatibles, et qu'il continue à espérer que personne ne s'en aperçoive. Ce phénomène, à lui seul, est tout à fait extraordinaire, pratiquement invraisemblable, mais ce n'est encore rien, car personne ne semble en effet s'en apercevoir, ou en tout cas s'en formaliser. Sauf, comme Pascal, pour polémiquer et persuader autrui des vertus de sa propre morale.

J'ai l'air de me moquer mais je ne me moque pas. Je répète au contraire qu'il ne semble pas en notre pouvoir de rompre le cercle de notre particularité morale. Je n'insiste que pour montrer à quel point le cercle est infranchissable. Et j'ajouterai que ce que je dis s'applique à ce que je dis, l'auteur de ces lignes est comme quiconque et n'échappe pas à cette règle. Comprendre la morale demande donc que l'on comprenne comment il se fait que non seulement la morale qui se présente comme universelle est en fait particulière, mais aussi pourquoi elle ne prend note de l'existence des autres que pour les déprécier. Je veux savoir pourquoi il n'est pas en notre pouvoir d'outrepasser cette méconnaissance. 


\section{Tableau 1}

\section{Liste des comportements du syndrome de l'ethnocentrisme}

Attitudes et conduites par rapport

au groupe

1.1 Voir son groupe comme vertueux et supérieur.

1.3 croire que ses propres standards sont universels, intrinsèquement vrais. Voir ses propres coutumes comme originales et au fondement de l'humanité.

1.4 Voir les membres de son groupe comme forts.

1.8 Des sanctions punissent le vol dans le groupe.

1.10 Des sanctions punissent le meurtre d'un membre du groupe.

1.12 Coopération avec les membres du groupe.

1.14 Obéissance aux autorités du groupe.

1.16 Empressement à demeurer un membre du groupe.

1.18 Empressement à se battre.
Attitudes et conduites par rapport à

l'extérieur du groupe

1.2 Voir l'autre groupe comme méprisable, immoral et inférieur.

1.5 Voir l'autre groupe comme faible.

1.6 Distance sociale.

1.7 Haine à l'égard de l'autre groupe.

1.9 Sanctions ou non sanctions du vol fait aux dépens des membres de l'autre groupe.

1.11 Sanctions ou absence de sanctions du meurtre d'un membre de l'autre groupe.

1.13 Absence de coopération avec les membres de l'autre groupe.

1.15 Absence d'obéissance aux autorités de l'autre groupe.

1.17 Absence de volonté de devenir membre de l'autre groupe.

1.19 Absence de volonté de se battre et de mourir pour l'autre groupe.

1.20 La mise à mort du membre de l'autre groupe est un acte vertueux.

1.21 Dans l'éducation des enfants on utilise le comportement des autres groupes comme exemple du mal.

1.22 L'autre groupe est tenu responsable des malheurs du groupe.

1.23 Méfiance et crainte à l'égard de l'autre groupe.

Reproduit de R.A. Levine at D.T Campbell. Ethnocentrism : Theories of Conflict, Ethnic Attitudes and Group Behaviour, New York, Wiley, 1972. Cité par Robert Boyd et Peter J. Richerson, "Cultural Transmission and the Evolution of Cooperative Behaviour », Human Ecology, vol. 10, $n^{\circ} 3,1982$. 
Il est temps de nommer ce phénomène. Je l'appellerai l'ethnocentrisme. Selon toute apparence l'ethnocentrisme est indépassable. La morale humaine est ethnocentriste. Le terme est emprunté à l'anthropologie (voir le Tableau 1): l'ethnocentrisme est la tendance à valoriser son propre groupe aux dépens des autres. Cette définition est circulaire: le défini (l'ethnocentrisme) est dans la définition (par le terme « valoriser »). Il n'est pas impossible de sembler échapper à cette circularité par des artifices de langage en décrivant le phénomène comme de l'extérieur ; on peut dire, par exemple, que l'ethnocentrisme est la croyance à la supériorité inhérente de toute morale, vue de l'intérieur de cette morale particulière. Mais ces artifices ne changent pas la chose ou la difficulté présente, ils ne réussissent qu'à masquer la réalité de l'ethnocentrisme du locuteur et son simple entêtement à refuser de la prendre en compte, ce qui est l'a b c de l'ethnocentrisme. Celui de Pascal, de G.E. Moore ou de tout locuteur.

Qu'on me permette d'insister. L'anthropologue qui nous dit qu'une société quelconque est ethnocentriste ne démontre pas de ce fait que lui-même n'est pas ethnocentriste, il montre seulement qu'il ne l'est probablement pas de la façon dont il parle à ce moment, mais qu'il l'est d'une autre dont il ne parle pas. J'en conclus que pour sortir d'un ethnocentrisme donne, pour le voir et le décrire, il faut se situer dans un autre ethnocentrisme qu'on né « voit » pas. Si nous disposions d'une seule anthropologie scientifique comme nous disposons d'une seule théorie de l'électricité, alors je pourrais dire que l'anthropologie scientifique échappe à tout ethnocentrisme. Mais puisque ce n'est pas le cas, il est raisonnable de penser que l'anthropologie comme science n'échappe pas à tout ethnocentrisme, qu'elle ne décrit l'un qu'à partir d'un autre.

De même, si je disais (I) que la morale est ethnocentriste, et (II) que ce que je dis n'est pas ethnocentriste, la proposition II invaliderait la proposition I et réaliserait par là la circularité en prétendant la rompre.

Pour dire ces choses, j'utilise le langage. Le langage est-il ethnocentriste ? Et comment ! c'est même un haut lieu de l'ethnocentrisme. C'est pour cela que lorsque nous traduisons un texte d'une langue dans une autre, nous reconnaissons qu'il y a toujours trahison : traduttore-tradittore, le traducteur (et la langue dans laquelle il traduit) ont toujours un lieu d'origine, contiennent et véhiculent une circularité indépassable.

Il devient nécessaire de reprendre l'ensemble de la question et de définir à nouveaux frais ce qui constitue l'essentiel de la « valeur », et par la même occasion la liberté humaine. L'entreprise ne va pas sans risque mais l'enjeu est important, impliquant possiblement les premiers éléments d'une nouvelle science de l'humain. 


\section{Chapitre 1}

\section{La morale et la liberté}

$\underline{\text { Retour à la table des matières }}$

La morale occupe de nos jours une place exceptionnelle dans l'ensemble de nos connaissances sur l'être humain. Depuis un siècle les sciences sociales ont peu à peu envahi un champ de connaissances jusque-là occupé par d'autres approches. C'est ainsi que sont apparues tour à tour l'économie, l'anthropologie, la sociologie, la science politique, la psychologie, et d'autres qui appliquaient la méthode scientifique aux affaires humaines. De nos jours, cette approche a envahi, ou fortement influencé, certains des domaines qu'on aurait pu croire parmi les moins propices à un traitement scientifique. Même la théologie et la critique d'art ont bénéficié, ou ont été influencées, par une meilleure connaissance de la science des matériaux, de meilleures méthodes de datation des artéfacts, par l'archéologie, la paléontologie, l'histoire et l'histoire économique, la linguistique. La morale, cependant, et je crois que son cas est maintenant unique, est demeurée indemne et non affectée par ce développement historique de nos connaissances sur l'être humain, vierge de toute approche positiviste ou de type objectif. Sans doute le cercle de l'ethnocentricité pose-t-il des difficultés particulièrement rébarbatives à une approche de ce genre.

L'existence de ces difficultés, et avec elles le caractère fortement « controversable » de tout discours sur la morale et sur l'ethnocentricité - dès qu'il est question de morale chacun de nous s'estime expert - nous amène, avant de proposer une définition de ce que l'on entendra ici par morale et par liberté, à stipuler des critères de définition, c'est-à-dire les conditions auxquelles chaque définition devra satisfaire pour être considérée acceptable. Les définitions sont libres, évidemment, à condition d'être cohérentes et leur usage constant. Ces critères sont donc l'énoncé des conditions auxquelles chaque définition est assujettie et qui en limitent l'usage. Ces critères sont au nombre de deux. 


\section{Observabilité}

La morale sera considérée comme un comportement, par conséquent comme un phénomène observable et éventuellement susceptible d'un traitement scientifique.

L'objectif évident de ce premier critère est, d'entrée de jeu, d'exclure de la définition toute référence à ce qui peut être dit «bon», "mauvais », à « la nature », à « la nature des choses », à « la nature humaine », à un « dieu » et à toute autre notion « inassignable » de ce type, c'est-à-dire ces notions qui ne sont pas des concepts construits par d'autres concepts mais des idées plus ou moins arbitraires ou locales ne renvoyant pas directement à des comportements observables. Ce qui retient notre attention n'est donc pas que la morale est intuitivement connue et approuvée comme telle ou non, mais que l'effet en est observable et donc vérifiable par quiconque et potentiellement prévisible.

Il faut prendre acte du fait que l'ensemble de la tradition philosophique occidentale s'objecte fortement, par principe, à une telle approche de la morale. Cette opinion déborde largement le petit groupe d'auteurs se réclamant du titre de philosophes et de moralistes et semble partagée par la plupart des sujets humains en Occident, crédules aussi bien que savants. Selon cette conception, très généralisée au point de sembler pratiquement la seule à être reçue en Occident, la morale relève d'abord et avant tout d'un acte intérieur de l'«esprit » ou de l'« âme », une intention, un devoir ou un choix effectué à la suite d'une délibération consciente.

La première difficulté posée par cette acception est d'ordre sémantique. Il est certain que tant que l'on définit la morale comme un acte intérieur, cet acte n'est pas observable et cela va de soi. Sans vouloir nier que la vie morale puisse impliquer de telles opérations intérieures conscientes, ce qui serait assurément stupide, on peut aisément constater que la moralité humaine produit aussi, par ailleurs, des effets universellement observables dans le comportement humain. Tout être humain est un être moral, il ne se conduit pas "comme une bête ", comme on dit, et cela est observable.

Assurément, on peut s'attendre à ce qu'une conception qui définit la morale comme un comportement observable produise un savoir fort différent de celui que nous en avons par le sentiment intérieur et par l'introspection. Mais l'objection de principe qui s'opposerait à une telle façon de voir serait du même ordre que celles qui s'opposaient autrefois à la naissance d'une psychologie expérimentale. Le sentiment intérieur, l'émotion, disait-on alors, ne sauraient être mesurés de manière objective, il est impossible de donner sens à une proposition qui dirait qu'une personne est en colère à huit pour cent. Ce qui est vrai. Encore aujourd'hui nous ne savons toujours pas décrire l'émotion de manière objective, mais cela n'empêche pas l'existence d'une psychologie objective et expérimentale qui rend d'immenses services. 


\section{Universalité}

Les définitions devront être universelles. C'est le second critère qui en limite la validité. Cette fois l'objectif visé est d'écarter toute conception morale particulière à telle ou telle tradition culturelle ou religieuse, société ou ethnie, ou à une période historique donnée.

Ce second critère est empirique. L'universalité dont il s'agit n'est d'aucune façon une loi en quelque sens que ce soit, ni un principe, ni même une règle au sens habituel de ce mot. Il s'agit plutôt d'une généralisation empirique comme on dit en science, c'est-à-dire d'un ensemble d'éléments de comportement très généralement observables chez les humains et auxquels on ne connaît pas d'exception importante ou le moindrement généralisée. Cette universalité est donc constatée empiriquement, c'est une donnée de l'expérience, et celui qui allègue l'existence d'un tel comportement universel le fait à ses risques puisque toute exception un peu importante peut la remettre en question ou demande à être expliquée. On ne dira pas, par exemple, que la morale humaine veut que le mariage humain soit monogame puisqu'il existe, notamment, plus d'un milliard de musulmans de par le monde qui sont ou peuvent être polygames.

La portée de ce second critère est plus étendue qu'il peut sembler à première vue. Il nous oblige à écarter, dans la définition de ce qu'est la morale humaine, toute la partie de la morale occidentale qui est basée sur les droits de la personne ou qui les exprime, et notamment la déclaration anglaise des droits de 1689, la déclaration américaine d'indépendance de 1776 et la déclaration française de 1789. Car c'est une conception strictement occidentale qui confère à la morale humaine une base individuelle et égalitaire. Cette conception est totalement étrangère à celle que l'on trouve dans l'Ancien Testament. Ce fait, à lui seul, est dirimant, il constitue un obstacle décisif. Cette conception est aussi étrangère à plusieurs populations autochtones américaines. Et je soupçonne qu'il en va de même pour la tradition de l'Islam et pour la pensée traditionnelle de l'Inde, de la Chine et du Japon classiques, et probablement d'un grand nombre de conceptions morales des sociétés primitives ou préindustrielles. La morale égalitaire et personnelle appartient à l'ethnocentrisme occidental, elle est relativement récente dans l'histoire humaine et date du XVIII ${ }^{\mathrm{e}}$ siècle. Elle ne nous est donc d'aucun secours ici parce qu'elle n'est pas universelle. Il nous est tout aussi impossible de fonder une définition objective de la morale sur l'ethnocentrisme occidental que sur celui, disons, de la société juive ou chinoise.

Dans ce qui suit, chaque fois qu'il sera question d'une morale non basée historiquement sur les droits de la personne, une telle morale sera dite basée sur le groupe. Ce sujet fera l'objet d'une étude du chapitre 3 qui traitera de l'importance du groupe. 


\section{Une définition de la morale}

La morale est un ensemble de contraintes. Une contrainte est un obstacle à quelque chose. Nous verrons plus loin, par la définition du concept de liberté, que la morale fait obstacle à l'exercice de certaines libertés.

Il convient d'élaborer ici ce concept de contrainte dont il sera fait longuement usage dans le présent ouvrage. En français le premier sens du verbe contraindre est " exercer une action contraire ; empêcher de suivre son cours » (Le Petit Robert). C'est ce sens associé d'abord et avant tout à l'idée de force que j'entends par contrainte morale, plutôt que l'idée de violence qui est le premier sens habituel du mot contrainte. La contrainte morale est ainsi une force qui nous oblige.

La contrainte dont il est question est biologique, opposée en ce sens à déterministe ou mécanique. La biologie est la science de la vie. Pour fixer les idées on peut illustrer la nature de ce genre de contraintes biologiques en citant la plus connue d'entre elles, celle qui fut décrite par Thomas Robert Malthus et qui devint par la suite la base de la théorie de l'évolution de Charles Darwin dans L'Origine des espèces en 1859 : le nombre des candidats à la reproduction excède toujours les ressources disponibles, ce qui entraîne une lutte pour la reproduction. On aura recours ici à la métaphore développée par Richard Dawkins dans Le Gène égoïste ${ }^{1}$ : si la population d'Amérique latine de 300 millions d'habitants continuait de s'accroître au rythme actuel, il faudrait moins de 500 ans pour que sa population doive se tenir debout en rangs serrés épaule contre épaule et couvre ainsi d'un tapis humain la totalité du continent ; dans 1000 ans, cette population sud-américaine formerait une pyramide de personnes, les pieds des unes reposant sur les épaules des autres, un million de personnes de haut ; dans 2000 ans cette montagne de personnes croîtrait à la vitesse de la lumière et aurait atteint les limites de l'univers connu. Voilà ce qu'est une contrainte biologique. Bien qu'elle n'opère pas par voie mécanique, il est certain qu'elle prévaudra, quelle que soit la politique démographique humaine, nataliste ou au contraire de planification des naissances.

\section{La contrainte morale}

Le concept de contrainte morale peut être construit sur le modèle du concept de compulsion utilisé en psychiatrie, qui est : type de conduite que le sujet ne peut s'empêcher d'adopter sous peine d'angoisse. Nous dirons de même, dans le domaine moral, que la contrainte morale affecte un ensemble de conduites que le sujet est porté à accomplir ou au contraire à éviter sous peine d'angoisse, de remords et de culpabilité.

\footnotetext{
1. Richard Dawkins, Le Gène égoïste, p. 161-162.
} 
Voici trois exemples simples. La plupart des humains, lors de leur premier contact physique avec leur nouveau-né, subissent une contrainte dont l'effet est de les porter à nourrir et protéger cet enfant. Cette contrainte est vérifiable, la plupart du temps, chez la plupart des gens. Une autre contrainte, inhibitrice celle-là, fait que la plupart d'entre nous, la plupart du temps, éprouvons une nette répugnance à commettre l'inceste. Troisième exemple, la plupart d'entre nous sommes particulièrement attentifs au bien-être et aux souffrances des membres de notre famille immédiate : nous leur accordons un traitement privilégié par rapport à celui que nous réservons à nos autres relations humaines. À l'intérieur d'un petit groupe dont le centre est la famille immédiate composée des parents et des enfants, nous sommes altruistes, prêts à faire des sacrifices, et cet altruisme décroît sensiblement à proportion de l'éloignement des bénéficiaires éventuels du petit groupe dont la famille immédiate est le centre.

Cette conception de la morale comme série de contraintes est certes différente de la conception traditionnelle en Occident qui en fait un choix personnel du sujet, délibéré et conscient, ou même comme l'acception d'une liberté conçue comme une forme qui serait sa propre loi, à la façon du rationalisme de Kant. Sur cette conception de la morale comme choix, je ferai quatre remarques. La première, c'est que son caractère introspectif ou théorique la rend nécessairement invérifiable dans la pratique. J'estime préférable de définir la chose dont il est question par les effets observables qu'elle produit.

La seconde remarque, c'est qu'on n'a pas dit grand-chose de la morale quand on a dit qu'elle est un choix. Car un choix implique l'exercice d'une préférence, ce qui suppose un critère de ce choix. Que choisit-on, si l'on choisit ? Il n'est pas raisonnable d'affirmer que la morale humaine est faite de choix si on laisse ensuite sans réponse la question de savoir quoi choisir. Et il n'est plus possible de répondre avec la naïveté du moraliste occidental qui nous réfère aux droits de la personne ou à la tradition rationaliste. Car nous savons que cette réponse est ethnocentriste et que cette morale n'est pas la morale humaine toute entière mais seulement la morale d'Occident. Nous savons en outre que cette apparente naïveté recouvre en fait une constante intolérance, vérifiable par la grande ignorance dont elle fait preuve à l'égard des autres sociétés humaines. En d'autres mots, la théorie du choix, affirmée sans plus de détails sur la nature de ce choix, est primaire ; une fois qu'on la pourvoit des précisions requises, elle devient de l'ethnocentrisme.

Par exemple, l'autorité que l'on pourrait reconnaître au précepte affirmant que « La morale, ce n'est pas le droit » dépend, d'une part, de ce que l'on entend alors précisément : de quelle morale s'agit-il, et aussi de quel droit (romain, napoléonien, ou au sens kantien) ? Mais en second lieu et plus encore, c'est le précepte luimême qui fait difficulté ou n'est pas universellement recevable, car ce précepte bien reçu à Paris ne l'est plus du tout à Téhéran ou dans les nombreuses autres capitales où la morale qui prévaut est celle qui est énoncée par le droit coranique. D'un point de vue occidental théorique, la morale qui prévaut à Téhéran ne diffère 
en rien du droit. En pratique, c'est toute la conception morale occidentale séparant radicalement la morale et le droit qui apparaît en cela insupportable à un grand nombre de sociétés humaines.

Troisième remarque. On ne peut nier que la vie morale des gens implique parfois un choix délibéré et conscient, mais il semble que de tels choix soient plutôt exceptionnels, rares ou clairsemés. Le moraliste qui soutient que la morale humaine est fondamentalement un choix délibéré et conscient a le loisir de s'interroger lui-même sur cette question : quand a-t-il, pour la dernière fois dans son passé immédiat, amorcé ou interrompu de cette façon une action en cours pour délibérer: «Dois-je choisir A ou B ? Dois-je faire telle chose ou dois-je au contraire m'abstenir d'intervenir dans les circonstances actuelles ? » S'est-il posé une telle question hier ? Avant-hier, peut-être ? La semaine dernière, alors ? Étaitce la semaine d'avant, le mois dernier, ou l'année dernière?

Ce genre d'introspection nous convainc ordinairement, pour ce qu'elle vaut, que de telles décisions morales prises à la suite d'un examen conscient et délibéré existent bel et bien mais sont relativement rares. Or, puisque la moralité de l'existence humaine est aussi ininterrompue que la vie elle-même - l'être humain est un être moral par définition, "à temps plein », pour ainsi dire, et non pas «à temps partiel » - ne faudrait-il pas supposer l'existence de quelque système permanent de moralité à l'intérieur de nous-mêmes, qui nous épargne habituellement d'avoir à prendre de telles décisions conscientes et délibérées tout en nous laissant le loisir de le faire dans certaines circonstances exceptionnelles?

Il y a du vrai dans une telle supposition. J'ai dit que la morale est une série de contraintes. Pour le mieux voir, on peut la comparer à une autre contrainte mieux connue, celle de la gravitation. À chaque instant notre corps est soumis à la contrainte gravitationnelle dont nous n'avons pas à nous préoccuper constamment parce que divers systèmes physiologiques, comme celui qui est logé dans l'oreille interne, informent à chaque instant le corps et assurent son équilibration. Il arrive cependant que certaines tâches particulières puissent requérir une évaluation consciente et un choix attentif de notre part. On rencontre de telles circonstances lors du lavage de fenêtres à grande hauteur, qui demande des précautions particulières, ou lors de promenades en forêt pour traverser un ruisseau sur des cailloux instables ou sur un tronc d'arbre glissant. Dans ce genre de circonstances particulières nous devons procéder à une évaluation consciente de la situation et agir avec précaution, probablement parce que les systèmes corporels automatiques qui assurent habituellement notre équilibre sont alors insuffisants et incapables d'assurer le fine-tuning requis par la situation particulière. Et cependant, malgré la réalité des choix que nous effectuons alors, nous ne disons pas que la gravitation est une question de choix mais une contrainte. Et c'est parce que cette contrainte est une constante de l'action humaine qu'elle exige parfois, mais pas toujours, une évaluation in situ et un choix conscient. Il en va de même dans le domaine propre de la morale, qui est une contrainte constante requérant parfois, mais pas toujours, 
une évaluation délibérée et un choix conscient. La morale n'est pas d'abord et avant tout une question de choix, c'est une contrainte.

Quatrième remarque. D'un point de vue épistémologique, l'un des grands inconvénients de la théorie de la morale comme choix est de rendre impossible une compréhension articulée des «bons » et aussi des «mauvais » choix au moyen d'un seul cadre théorique. Pourquoi, en effet, certaines personnes choisissent-elles « mal » ? Et que font-elles au juste quand elles choisissent « mal » ? Je veux dire que même si l'on supposait par exemple, pour les fins de la présente discussion, qu'une «bonne » action est celle qui est conforme a un code reconnu tel les droits de la personne, quel rapport y a-t-il entre ces droits et les motifs qui sont à l'origine du « mauvais » choix ? Nous disons qu'une porte peut être ouverte ou fermée et cela est intelligible parce que dans les deux cas il s'agit de la même porte ; si une action peut de même être dite «bonne » ou "mauvaise », qu'y a-t-il de commun aux deux actions?

Nous sommes un peu dans la situation des contemporains de Newton qui alléguaient une raison pour rendre compte de la chute des corps («l'horreur du vide »), et une autre toute différente pour expliquer l'ascension d'autres corps comme la flamme et la fumée. Nous savons que lorsque nos explications contiennent autant de principes qu'il y a de phénomènes à expliquer, nous ne sommes pas très éloignés du pur verbalisme de la vertu dormitive de l'opium. Nous aurions donc besoin d'une approche unifiée et englobante, d'un cadre théorique unique assez vaste pour accueillir l'ensemble des phénomènes impliqués, non seulement la morale proprement dite mais aussi l'immoralité. C'est ce que nous fournira le concept de liberté.

\section{Définition de la liberté}

J'appelle liberté l'ensemble des performances dont un organisme est physiquement capable. C'est l'autonomie de l'organisme dans son environnement.

Cette définition doit être entendue littéralement et empiriquement. La liberté d'un être humain inclut la possibilité de parler, de marcher, de courir, ce sont là autant de performances qui lui sont possibles, mais non pas de voler dans les airs comme un oiseau ni de vivre sous l'eau comme un poisson car sa conformation physique ne permet pas ces performances. Un chien peut de même marcher et courir, mais ne peut ni parler, ni voler dans les airs, ni vivre sous l'eau, son corps ne le permettant pas. Toute liberté est d'abord physique et prédicable à tout organisme.

Revenons maintenant à la morale, comme ensemble de contraintes, pour identifier sa place et son rôle par rapport à la liberté. La liberté est un ensemble que la morale, comme sous-ensemble, vient réduire. La morale est un sousensemble de contraintes dont l'effet est de diminuer, circonscrire et limiter la 
somme des performances qui, bien que physiquement possibles, deviennent en pratique soit obligées, soit au contraire, inhibées. L'effet de la contrainte morale peut être incitatif ou inhibiteur ; elle peut nous obliger à faire quelque chose que nous pourrions ne pas faire, ou au contraire nous inhiber et ainsi nous empêcher de faire quelque chose que nous pourrions faire physiquement.

Reprenons, pour le voir, les trois exemples de contraintes morales cités précédemment. J'ai dit qu'un être humain, lors de son premier contact physique avec son nouveau-né, subit une contrainte dont l'effet lui fait nourrir et protéger cet enfant. En tant qu'être libre, cet humain a la possibilité matérielle, physique, de nourrir et protéger, ou au contraire de s'en abstenir et de se désintéresser de cet enfant ; cette dernière possibilité existe. Mais parce que cet être humain est un être moral il est contraint, et la possibilité matérielle qu'il a de ne pas nourrir et protéger son enfant ne sera pas mise en pratique. La morale est une contrainte, un obstacle, une entrave à l'exercice d'une liberté.

Il en va de même dans le cas de l'inceste. Physiquement, un être humain peut s'engager ou ne pas s'engager dans une conduite incestueuse. Dans les faits et dans l'immense majorité des cas, il n'en fera rien parce que la contrainte morale qu'il subit est trop forte et l'empêche d'exercer la liberté qu'il a sur ce point, la neutralise et l'inhibe.

De même encore dans l'altruisme. Un être humain a la possibilité matérielle de secourir en priorité des étrangers en détresse et requérant son aide avant de porter secours à sa famille immédiate. D'habitude il n'en fera rien parce que la contrainte morale s'avérera trop forte, il accordera priorité au bien-être de ses parents et de ses enfants, à ses propres dépens et à l'occasion au risque de sa vie. La contrainte morale prévaut habituellement et le prive de l'exercice de la liberté physique qu'il a pourtant de privilégier un étranger plutôt qu'un membre de sa famille.

Pourquoi définir la liberté comme ensemble de performances physiques de l'organisme ? Une définition de ce genre apparaît inévitable si l'on se rappelle les deux critères de définition mentionnés plus haut. Selon le critère d'observabilité, la présente recherche ne concerne pas l'intuition que l'on peut avoir de la liberté (sans pour autant vouloir en rien nier l'intérêt d'une telle vision introspective), mais consiste plutôt à prendre acte des effets observables de cette liberté. S'il existe de tels effets observables, physiques, on doit supposer qu'il existe aussi des conditions de possibilités ou des antécédents de ces effets, lesquels devront eux aussi être de quelque façon physiques. C'est ce qui nous oblige à écarter diverses notions introspectives de la liberté comme : "La liberté, c'est le pouvoir de faire ce que l'on veut », car un tel " pouvoir » n'est pas observable ; «l'absence de contraintes extérieures » dont parle Thomas Hobbes dans le Leviathan ${ }^{1}$, d'abord parce que, au

1 Thomas Hobbes, Leviathan, p. 189. [Texte disponible dans Les Classiques des sciences sociales. JMT.] 
sens strict, une absence n'est pas observable, et ensuite parce que tout organisme humain étant astreint à la gravitation, ce qui est une contrainte, il n'existe pas de telle chose pour l'être humain qu'une vie sans contraintes. De même encore devons-nous écarter ici la liberté « contingente » de Sartre dans L'Être et le Néant, ou la « corvée » dont parle Camus dans Le Mythe de Sisyphe, et autres produits de l'introspection qui ne sont pas observables.

Le deuxième critère, l'universalité, nous fait écarter toute conception de la liberté qui suppose le sujet. On rappellera pour mémoire qu'il n'y a pas de concept de sujet chez Aristote. Le sujet est une invention typiquement moderne occidentale, elle n'apparaît que dans la tradition philosophique occidentale chrétienne, ses premiers représentants historiques étant Augustin, Descartes et surtout Kant. L'idée de sujet n'est pas universelle mais seulement occidentale et donc ethnocentriste.

Néanmoins, cette idée de sujet est si importante dans l'histoire des idées en Occident, dans son rapport à l'idée de liberté, qu'elle mérite ici une discussion particulière. Toute la philosophie occidentale, depuis Kant, a été et demeure encore à ce jour marquée par l'apparente réversibilité ou convertibilité de ces deux termes que sont, d'une part, la liberté et d'autre part, la raison ou la pensée qui est la première caractéristique du sujet. Pour nous tous, en Occident, la liberté et la pensée semblent être en pratique la même chose : la liberté tend à se confondre avec la pensée et la liberté de la pensée avec la pensée de la liberté.

Peut-on sortir de ce cercle constitutif de l'ontologie occidentale moderne et de la métaphysique du sujet qui associe l'idée de liberté, idée déjà bien assez mystérieuse par elle-même, à cette autre idée de la pensée qui est peut-être plus mystérieuse encore que l'idée de liberté ? Historiquement, l'invention kantienne du sujet humain qui est sa propre liberté et qui, en cela, est donc égal à tout autre sujet humain, était d'une extraordinaire audace à l'époque puisqu'elle s'adressait à des hommes et à une philosophie d'Ancien Régime pour lesquels elle fut exactement ce qu'elle voulait être : une rupture démocratique et égalisatrice qui mettait fin à la hiérarchie métaphysique et théocratique. C'est ce qui, en philosophie, fait de Kant le véritable père des droits de la personne. Cette philosophie était révolutionnaire en son temps mais il y a belle lurette qu'elle ne l'est plus pour nous qui sommes post-révolutionnaires et pour qui la réversibilité de la liberté et de la pensée est devenue en fait beaucoup plus cartésienne que kantienne. Nous continuons en effet à croire, malgré toute la psychanalyse que l'on voudra, que tout ce qui est dans la liberté humaine est dans sa pensée et réciproquement. C'est l'âme transparente à elle-même de Descartes qui ne pense que ce qu'elle est et qui pense n'être que ce qu'elle pense qu'elle est. Nous devrons donc retourner brièvement à Kant pour reprendre les données de ce problème afin de le reformuler pour notre compte et le résoudre en termes modernes. 
La formulation kantienne de ce problème se trouve dans la troisième Antinomie ${ }^{1}$ où Kant expose le conflit qu'il estime irréductible et insoluble entre la causalité naturelle au sens de l'univers de Newton et la causalité au sens de David Hume d'une part, et la « causalité libre » produite par le sujet, d'autre part. Si la chaîne de la causalité naturelle des choses existe, on doit la concevoir comme sans fissure et il n'y a aucune place dans le monde pour quelque chose ressemblant à un sujet. C'est cette scission radicale des choses et du sujet effectuée par Kant qui est probablement le plus important événement dans l'histoire du subjectivisme occidental, qui persiste encore dans notre philosophie actuelle et que nous retrouvons, pratiquement intacte, dans la vénérable mais à ce jour encore réputée insoluble opposition du déterminisme et du libre arbitre. On la retrouve encore, par exemple, dans l'article « Free Will » de l'Encyclopœdia Britannica paru en 1959 et rédigé par John David Mabbott de l'Université Oxford, qui conclut son texte en faveur de l'indéterminisme et du libre arbitre.

Pourtant, du point de vue descriptif limité à l'observabilité qui est le nôtre, le problème est loin d'être insoluble, il est même relativement simple. Il suffit, pour le voir, d'identifier précisément les termes du débat dans les termes de la science actuelle. C'est ce que nous ferons maintenant.

\section{(1) Déterminisme \\ Libre arbitre}

Si l'objectif de la démonstration est de définir la liberté traditionnellement identifiée à la pensée ou à « l'arbitraire » ou à la " causalité libre » du sujet, il convient en premier lieu de trouver un équivalent fonctionnel du terme de liberté, c'est-à-dire une autre expression qui véhicule le même sens. Le terme d'indéterminisme convient à ce rôle. Nous pouvons donc transformer la proposition I en la proposition II :

\section{(II) Déterminisme}

VS

Indéterminisme

L'opération suivante consiste à nous demander ce que signifie pour nous de nos jours le déterminisme et surtout ce qu'il a signifié dans le passé pour ceux qui l'opposent encore à l'indétermination présumée de la liberté humaine. C'est essentiellement l'univers de Newton et des trois lois de Kepler. La révolution copernicienne effectuée par Kant transpose en philosophie la description de l'univers fournie par Newton : c'est une mécanique dont tous les moments sont prévisibles, dans l'avenir comme dans le passé. Et c'est ce que l'on a traditionnellement appelé le déterminisme: une chaîne causale naturelle sans faille, absolue, dans laquelle tout est donne avec les conditions initiales et où il n'y a aucune place pour le sujet. C'est sur ce déterminisme illimité que la mécanique du siècle dernier s'est édifiée.

\footnotetext{
Immanuel Kant, Critique de la raison pure, p. 348-9.
} 
Qu'en est-il aujourd'hui ? Il y eut tout d'abord la thermodynamique qui vint ébranler ce déterminisme. Puis une définition statistique de l'entropie associa l'ordre au non-équilibre. Le langage scientifique s'est transformé en conséquence. Là où l'on voyait autrefois des causes, on parle dorénavant de probabilités et l'univers décrit par Newton est maintenant dit d'une très haute probabilité. Ce très hautement probable est le propre de l'entropie qui indique la direction de la « flèche » du temps.

Le problème philosophique traditionnel s'en trouve donc transformé lui aussi. Pour l'observateur que nous sommes, il n'y a pas de différence observable entre les effets produits par la " causalité naturelle » et ceux de la "causalité libre » que Kant leur opposait, il n'y a pas davantage deux concepts distincts qui seraient à l'œuvre mais seulement un seul, celui de la causalité au sens de Hume, successivement affirmé absolument dans le premier terme et nié absolument dans le second terme. Par conséquent, lorsqu'elle est interprétée dans le cadre de la théorie des ensembles, cette ancienne opposition absolue entre déterminisme et indéterminisme prend la forme du type d'opposition existant entre un ensemble et son complément : tout ce qui est affirme absolument de l'un est nié absolument de l'autre. Si donc ce que l'on appelait autrefois le déterminisme s'appelle maintenant en jargon moderne une très haute probabilité, qui est l'entropie et le désordre croissant dans l'univers, son complément ne peut être qu'une très faible probabilité et l'ordre croissant de l'entropie négative. C'est-à-dire la vie. Ce qui donne :

\section{(III) Désordre probable vs Ordre improbable, Entropie \\ Entropie négative, Vie}

Il n'y a rien là de très neuf. C'est pratiquement depuis l'apparition du concept d'entropie, au milieu du siècle dernier, que l'on sait que la vie est une forme d'entropie négative, d'un ordre croissant et improbable.

On conclura que tout ce que l'on désignait confusément jusqu'ici par les termes de liberté et de libre arbitre, et autres semblables, appartient en fait à la vie. Et il est bien vrai que le désordre croissant fortement probable de l'entropie a apparemment « engendré » un sous-système qui lui est à la fois intégré et malgré tout opposé, l'ordre croissant de l'entropie négative de l'organisme vivant, l'improbable.

Il ne faudrait pas croire que cette définition de la nature de la liberté mette un point final au problème du déterminisme et du libre arbitre. Cette formulation n'est acceptable qu'au regard et dans des termes admissibles Pour la science. Car d'un point de vue traditionnellement humaniste ou philosophique, il serait aisé de trouver des arguments tout aussi convaincants pour résoudre le problème en faveur du libre arbitre et conformes à l'argumentation de John David Mabbott. C'est ce 
que Kant visait lorsque, dans la troisième Antinomie de la raison pure, il décrivait le problème engendré selon lui par la conception humienne de la causalité ${ }^{1}$. Kant a montré qu'il y a plus qu'un point de vue légitime sur les questions de ce genre, et que certains de ces points de vue peuvent être irréductibles comme dans le cas de la causalité. Nous conclurons pour notre part que puisque ni l'un ni l'autre de ces points de vue ne parvient à déloger l'autre ni n'abandonne le terrain, on peut supposer que les deux nous apportent une information importante sur la pensée humaine et sa réalité. On n'affirmera donc pas que le « libre arbitre » n'existe pas mais plutôt, ce qui est très différent, que c'est une de ces choses que je ne saurais expliquer dans le cadre du discours cohérent, ce qui est en vérité banal.

En revanche cette interprétation rend justice à la position de G.E. Moore ${ }^{2}$ concernant l'erreur naturaliste (naturalistic fallacy) de toute tentative de passer logiquement d'un jugement de fait a un jugement de «valeur », puisque toute proposition contenant un jugement de «valeur » peut être rapportée à l'exercice d'un libre arbitre. Dans les sciences sociales il est relativement aisé de citer des auteurs soutenant la même opinion. L'anthropologue V. Reynolds ${ }^{3}$, par exemple, déclare sans ambages qu'il est " tout simplement impossible de faire dériver la morale de la biologie ».

De plus, cette position définissant la liberté comme la vie elle-même ne doit pas être appelée « naturaliste » au sens interdit par la doctrine de G.E. Moore ni, en termes plus généraux, matérialiste. On pourrait même prétendre que la doctrine matérialiste est logiquement insoutenable dans la mesure où ce terme désigne une théorie qui dit avec des mots que les mots sont sans importance et que seule la matière importe.

L'intérêt d'une conception de la liberté exprimée en termes de vie peut maintenant être résumé en trois points :

1. La liberté devient une réalité conceptualisable et accessible à l'analyse comme tout autre objet de pensée. Ce qui rend la liberté humaine pensable est le fait de l'inclure dans une catégorie plus grande qu'elle-même qui est la vie. Tant qu'elle avait le statut d'une causalité « libre » ou incausée, l'idée de liberté humaine demeurait impensable, inanalysable parce qu'elle n'était pour ainsi dire " pas de ce monde » et en pratique très proche de l'idée que l'on peut avoir de Dieu. Le cogito de Descartes ou le sujet kantien relève d'une sorte de théologie à usage humain, de divinité faite philosophe radicalement inassimilable aux autres objets connus. Lorsqu'elle est au contraire exprimée en termes d'entropie négative, la liberté devient accessible à une compréhension

\footnotetext{
Immanuel Kant, Critique de la raison pitre, p. 348.

G. E. Moore, Principia Ethica, p. 10.

V. Reynolds, V., The Biology of Human Action, p. 34.
} 
scientifique ordinaire, par exemple, comme partie d'un système autorégulateur ${ }^{1}$.

2. La liberté humaine, bien que très grande, est, à tout moment historique donné, finie. Elle est limitée d'une part par l'existence de la morale qui vient en circonscrire l'exercice. D'autre part, elle est aussi liée à l'architecture du cerveau humain, dont on nous dit certes que c'est la chose la plus complexe de l'univers, mais dont il faut tout de même supposer que la complexité demeure, de quelque façon, finie. Néanmoins, elle est en même temps potentiellement infinie ou sans limite assignable dans l'avenir, et cela pour trois raisons :

Premièrement, les autres organismes vivants subissent l'évolution, alors que l'être humain s'oppose, parfois de façon victorieuse notamment par la médecine, à certaines des conséquences de l'évolution. La science lui permet, dans une certaine mesure, de prendre en main les clés de cette évolution. Les générations passées se posaient la question : «Qu'est-ce que l'être humain ? », la nôtre commence à se demander ce que nous voulons qu'il devienne.

Deuxièmement, la liberté humaine est aussi potentiellement sans limite assignable parce que la technique humaine augmente sans cesse le nombre et la variété des performances qui nous sont désormais physiquement possibles, comme voler en avion ou communiquer à distance, performances qui étaient impossibles à nos prédécesseurs.

Troisièmement, l'accroissement de la liberté est aussi possible par la diminution de l'importance relative des performances que la morale contrôle, impose ou inhibe. On sait, par exemple, que les formes concrètes que prend l'interdit de l'inceste varient beaucoup d'une société à une autre. Dans une société primitive ne regroupant qu'un petit nombre d'individus, les règles du mariage sont positives et négatives et peuvent n'offrir à chaque candidat au mariage qu'un nombre très restreint de conjoints possibles, alors que dans les sociétés industrielles à forte densité de population il suffit de quelques règles négatives peu contraignantes pour parvenir au même résultat. L'impératif de l'interdit de l'inceste demeure partout présent, mais son application peut varier, et ainsi accroître ou au contraire diminuer l'exercice des libertés individuelles. On peut même imaginer pour l'avenir la suppression progressive de cet interdit moral de l'inceste, (ce que je ne suggère pas), qui a toujours été vécu jusqu'ici comme un destin, puisque les moyens contraceptifs modernes nous donnent le loisir d'en supprimer les inconvénients génétiques s'il en est, ce qui accroîtrait d'autant l'autonomie relative des individus. Mais encore faudrait-il, si l'on voulait avoir accès de façon systématique à un tel accroissement de la marge de manœuvre des initiatives individuelles, connaître les bases biologiques de la

Les sciences sociales, et c'est nouveau, commencent à s'intéresser au concept d'entropie. Voir Michel Forsé, L'Ordre improbable. 
morale humaine, ses contraintes et ses limites. C'est ce que nous tenterons de faire voir à l'instant.

3. Mais le plus grand intérêt, et de beaucoup, d'une conception de la liberté qui en fait une caractéristique de la vie apparaît lorsqu'on se rappelle que chaque être humain est biologiquement unique. Le nombre de combinaisons possibles du génome humain est si élevé que nous n'avons pas d'expression mathématique apte à en faire voir aisément toute l'extraordinaire richesse. Il y a dans ce fait la base factuelle d'un prodigieux potentiel de variété de vie qui nous aide à comprendre un peu mieux comment l'humanité a pu progresser comme elle l'a fait jusqu'ici et combien elle peut progresser encore dans l'avenir si l'on tente, en connaissance de cause, de favoriser l'épanouissement de chaque individu biologiquement unique.

\section{Les origines biologiques de la vie sociale}

Voyons maintenant la question de l'origine biologique de la morale. S'il est vrai que la morale est un ensemble de contraintes venant limiter les performances des individus, nous devons tout d'abord nous efforcer d'identifier l'origine de ces contraintes. Dans la mesure où cette origine est sociale, il importe de comprendre comment la biologie interprète le fait social. Pourquoi sommes-nous sociaux ?

Il faut nous référer à Darwin. Tout d'abord une remarque générale sur la théorie évolutionniste utilisée ici, sous forme forcément très simplifiée, de la sélection naturelle de Darwin, complétée par la « Synthèse moderne » des années 1930 et par la « Nouvelle synthèse » d'Edward O. Wilson de 1975. Aucun compte ne sera tenu des tentatives récentes comme celle de Stephen Jay Gould sur l'évolution «par sauts » ${ }^{1}$, la critique de l'« hypersélectivisme » par Richard Lewontin ${ }^{2}$ et autres contributions semblables. La raison en est que la nature propre de l'évolution est une question de fait et que ce n'est pas l'affaire du philosophe de prétendre trancher ce genre de questions lorsque les savants impliqués dans ce genre de débat éprouvent des difficultés à s'entendre sur ce qui constitue ce fait. On ajoutera seulement que selon William L. Brown Jr. ${ }^{3}$ les faits originellement présentés à l'appui de la thèse de l'équilibre ponctué ne la justifient pas.

L'évolution des vivants résulte de la sélection naturelle, environnementale, entre les candidats à la reproduction qui entrent en compétition pour l'utilisation des ressources insuffisantes pour eux tous. Dans le dernier paragraphe de l'édition originale de L'Origine des espèces, Darwin disait :

\footnotetext{
S. J. Gould et N. Eldredge, « Punctuated Equilibrium ». S. J. Gould et R. Lewontin, The Spandrels of San Marco and the Panglossian Paradigm. William L. Brown Jr., «Punctuated Equilibrium Excused: The Original Examples Fail to Support It ». Voir également la discussion de l'ensemble de cette question dans le chapitre 9 de L'Horloger aveugle de Richard Dawkins, p. 262-296.
} 
Le résultat direct de cette guerre de la nature, de la famine et de la mort, est donc le fait le plus élevé que nous puissions concevoir, à savoir, la production des animaux supérieurs ${ }^{1}$.

Dans quel sens sont-ils dits supérieurs ? L'interprétation de ce passage demeure ouverte, car Darwin se met en garde lui-même ailleurs dans son œuvre contre les risques inhérents à de telles comparaisons entre animaux dits "supérieurs » et « inférieurs ». Dans le cas présent, l'expression « animaux supérieurs » peut certes désigner les plus complexes, comme les humains, mais elle peut également signifier que les hirondelles présentement existantes sont les meilleures hirondelles possibles sur deux points : 1) par leur reproduction, c'est-à-dire le nombre de descendants que chaque couple d'hirondelles est apte à produire et mener a maturité dans la génération suivante ; 2) compte tenu de leur histoire génétique et des ressources disponibles dans leur niche dans l'environnement actuel. Ces hirondelles sont en lutte entre elles parce que les ressources de la niche sont insuffisantes pour toutes les hirondelles candidates à la reproduction, et elles sont aussi en lutte avec les autres organismes utilisateurs des mêmes ressources. En résumé, les animaux que Darwin appelle «supérieurs » sont le produit de la plus sévère sélection naturelle. En anglais on les appelle des survivors, autrement dit des vainqueurs dans la lutte pour la reproduction. Dans un langage plus technique on dira que l'évolution ne les a pas « décimés », selon l'expression proposée dans un autre contexte par S. J. Gould ${ }^{2}$.

Le tableau qui se dégage de cette description de Darwin est, à première vue, celui d'une lutte incessante de chacun contre chacun et de tous contre tous par la famine et la mort, dit-il. La « logique » de la compétition incline à l'élimination du concurrent. Il se trouve que deux cents ans avant Darwin le philosophe anglais Thomas Hobbes avait dressé un tableau analogue de l'humanité dans son Leviathan. Hobbes imaginait ce que devait être l'être humain d'avant le social. C'est ce qu'il a appelé l'« état de nature », la vie des humains avant qu'ils ne décident de vivre en société et n'inventent la vie sociale à eux tout seuls, pensait Hobbes. Dans cet état de nature il décrit ${ }^{3}$ " the life of man solitary, poore, nasty, brutish and short »: une vie humaine qui aurait été solitaire, misérable, vilaine, brutale et courte.

Hobbes, pas plus que Darwin, n'a observé personnellement un tel état de choses. Chacun présente un modèle, le premier pour rendre plausible la fable selon laquelle les hommes ont un jour inventé la vie sociale, le second pour comprendre l'évolution des vivants. Et dans un cas comme dans l'autre le tableau ne correspond

1 Charles Darwin, L'Origine des espèces au moyen de la sélection naturelle, p. 490. [Texte disponible dans Les Classiques des sciences sociales. JMT.]

2 Gould, Stephen Jay (1989). Wonderful Life. The Burgess Shale and the Nature of History, p. 47, note.

3 Hobbes, Thomas (1955). Leviathan, p. 185. [Texte disponible dans Les Classiques des sciences sociales. JMT.] 
à rien d'observable. En fait, en ce qui concerne la vie, tôt ou tard, d'une façon ou d'une autre, la vie finit toujours par trouver des compromis qui abaissent le niveau de la compétition caractéristique de "la guerre de chacun contre chacun». L'évolution favorise toujours, de multiples façons, les organismes qui se situent dans des stratégies de reproduction à compétition moins brutale ou moins éliminatoire pour les concurrents. On peut donc affirmer que la «logique » de la compétition tend à éliminer le concurrent, mais cela veut dire qu'en pratique la chose est déjà faite depuis longtemps dans tout biotope ou écosystème observable : l'une des premières découvertes dans la courte histoire de l'écologie est la règle énoncée par Gause en 1934 selon laquelle deux espèces différentes ne peuvent compétitionner (ou subsister) dans la même niche. En d'autres mots, dans tout système observable, l'« état de nature » décrit dans la fable de Thomas Hobbes est un état dont la nature elle-même ne semble pas vouloir. C'est l'une des raisons qui font que toute forme vivante observable est un animal supérieur, comme dit Darwin : elle est le résultat d'une stratégie évolutive de compromis. Le nombre et la variété de ces stratégies de compromis sont extraordinairement grands, non énumérables. La vie sociale est l'un de ces compromis.

\section{De l'origine du social}

Je sais que cette dernière affirmation, à l'effet que la vie sociale résulte d'un développement particulier et contingent de l'évolution darwinienne, risque de heurter un certain nombre de sociologues et d'anthropologues qui pourraient soutenir une opinion contraire. Il sera difficile d'en discuter tant que ces divers spécialistes ne nous donneront pas leurs raisons et ne nous procureront pas, en termes raisonnablement vérifiables, une réponse à la question suivante : quelle est selon eux l'origine de la vie sociale, par opposé à la vie solitaire?

Pour le philosophe, la première conséquence de la conception du social comme forme de vie produite par l'évolution est que ces deux modèles, celui de Hobbes et celui de Darwin, sont totalement incompatibles et s'excluent mutuellement. Si nous acceptons le modèle de Hobbes, alors Darwin a tort, nous sommes des enfants de Dieu et la théorie hobbesienne du «contrat social " appartient à la version protestante des suites de l'expulsion du paradis. Ce sont les humains et eux seuls qui ont inventé la société en renonçant à la totalité de leur liberté individuelle (au sens hobbesien d'absence de contraintes), donc en enchaînant leur liberté à l'absolutisme du Léviathan, qui est un despote. Un siècle après Hobbes, JeanJacques Rousseau a proposé une version plus latine, moins luthérienne de cette même fable. Il se trouve que les philosophes ne disposent d'aucune autre théorie de l'origine du social que cette fable.

Ou bien, au contraire, nous acceptons la théorie darwinienne de l'évolution, mais il nous faut alors être conséquents, ces choses-là ne doivent pas se faire à moitié, il faut en voir et en accepter intégralement les conséquences. Ce qui signifie que toute la théorie philosophique du « contrat social » doit être appréciée 
pour ce qu'elle est, c'est-à-dire une fable philosophique à forte saveur théologique. C'est l'évolution qui a inventé la vie sociale comme mode de compromis par rapport à la compétition, et non pas les humains par leur seul génie. Si loin que l'on remonte dans l'histoire de l'humanité et au-delà dans ce que l'on sait des anthropoïdes et des premiers représentants de l'espèce homo, les humains semblent toujours avoir été sociaux, aucun fait connu ne nous permet de croire que ce primate ait jamais été solitaire.

Cela mérite d'être dit plus explicitement encore. Il n'y a pas le plus infime document historique justifiant la thèse d'un « contrat social » prétendument conclu entre humains qui auraient vécu jusque-là dans un "état de nature » comme Rousseau et Hobbes voudraient nous faire croire. On demandera à savoir à quelle date ce supposé contrat a été conclu, quels en étaient les signataires et où sont les traces de leurs témoignages.

Reprenons donc à nouveaux frais l'examen de la question : pourquoi les êtres humains vivent-ils en société plutôt que solitaires ?

Dans son ouvrage Du contrat social ou principes du droit politique de 1762 Jean-Jacques Rousseau résume en ces termes, au chapitre VI, le problème posé :

Si donc on écarte du pacte social ce qui n'est pas de son essence, on trouvera qu'il se réduit aux termes suivants : Chacun de nous met en commun sa personne et toute sa puissance sous la suprême direction de la volonté générale ; et nous recevrons encore chaque membre comme partie indivisible du tout.

Je soutiendrai qu'indépendamment de la faiblesse souvent remarquée du concept de "volonté générale ", ce contrat ou pacte social étant une entreprise de langage ne peut en aucun cas créer la société humaine elle-même : le contrat ne peut être à l'origine d'une société qu'inventeraient de toutes pièces des êtres humains jusqu'alors solitaires. Demandons-nous, en effet, si les orignaux ou élans d'Amérique, Alces alces, du nord du Québec, qui sont pratiquement solitaires pourraient devenir sociaux au moyen d'un pacte social de ce genre. La réponse est évidemment négative puisque ces cervidés ne disposent pas du langage. Mais la réponse doit être tout aussi négative dans le cas des humains car le langage ne tombe pas du ciel. Partout où l'on trouve du langage, on est assuré de trouver une société déjà constituée depuis fort longtemps, utilisant le langage et le reproduisant. Le problème est donc le suivant : ou bien les hommes de l'« état de nature » possédaient déjà le langage, ou bien ils ne le possédaient pas ; s'ils ne le possédaient pas, ils étaient incapables de fonder la société humaine, s'ils le possédaient, la chose était déjà faite. Il faut éviter de confondre le social et l'État : logiquement et dans le temps le social précède le langage et donc aussi l'État. On reconnaîtra donc que la théorie du «contrat social » n'explique pas l'origine de la société humaine : elle répond à une question tout autre et n'explique au mieux que la forme locale de gestion sociale humaine à un moment donné de l'histoire. 
Le raisonnement est simple et minimaliste, il obéit au principe de parcimonie : puisque les abeilles sociales, les fourmis sociales et les babouins sociaux ont bien su se passer de « contrat social » pour développer leurs formes propres de vie en société, pourquoi en aurait-il été autrement pour les primates hominidés d'avant le langage qui nous ont précédés ? Et en quoi l'apparition ultérieure du langage humain aurait-elle modifié la donne de ce problème ?

En pratique, seule la biologie répond à la question de savoir pourquoi nous sommes sociaux : nous sommes sociaux parce que c'est ainsi que l'évolution nous a faits. La vie sociale est une stratégie, parmi d'autres, de l'évolution, dont l'effet présumé est de réduire l'âpreté de la compétition dans la reproduction.

Ce qui veut dire, revenant à notre problématique morale, que si l'on demande contre quoi nous luttons dans notre vie morale ou dans une science comme la médecine dont la pratique quotidienne fait une grande "consommation » de morale, il faut répondre que nous luttons contre certaines conséquences jugées indésirables de l'évolution; et si l'on demande en faveur de quoi nous luttons en morale, il faut répondre que nous luttons en faveur de certaines conséquences jugées désirables de l'évolution. S'il se trouve que le philosophe occidental qui continue à faire de la théologie sans le savoir insiste dans ses derniers retranchements actuels pour savoir d'où provient le langage, il faut lui répondre qu'à notre connaissance le langage est une invention, probablement la plus belle, de l'évolution de la vie. Pourquoi ? Parce qu'il n'y a pas d'autre réponse possible. Certes, l'origine du langage demeure hors de portée de la science évolutionniste actuelle, mais notre ignorance sur ce point n'autorise nullement une remise en question de ce que nous savons par ailleurs sur l'évolution. Hormis le créationnisme et jusqu'à nouvel ordre, nous ne connaissons aucune autre hypothèse raisonnable que celle qui fait du langage une production probable de l'évolution. C'est ce que signifie l'acceptation de la théorie de l'évolution dans toutes ses conséquences. Les philosophes d'Occident, en général, acceptent la théorie darwinienne, mais pas dans toutes ses conséquences. Ce qui n'est pas tout à fait une contradiction (laquelle, finalement, n'est pas tellement grave parce qu'éventuellement chacun se corrige soi-même ou une instance quelconque le rappelle à l'ordre), c'est une ambivalence. L'ambivalence est un état mental qui interdit la recherche et l'invention, et c'est dans ce jus que la philosophie morale d'Occident macère depuis 1859 et L'Origine des espèces.

Je conclus pour ma part que la société est une invention de la vie. C'est de la vie grand format ou écrite à grands traits. Ce qui ne signifie pas que la biologie comme science puisse par elle-même rendre compte de toute forme de vie, particulièrement dans le cas des êtres humains, mais plutôt qu'il est imprudent ou peu sage d'imaginer l'existence d'une ligne fixe et permanente qui séparerait le domaine de la biologie de celui des sciences sociales. De même qu'à l'heure actuelle et pour diverses raisons certaines oppositions massives comme celle de « inné/acquis » sont en voie de passer de mode dans le raisonnement en biologie, 
de même des dichotomies du genre : « Ou bien ce phénomène relève de la biologie ou bien c'est du social » passent à côté de quelque chose d'important qui est que tout ce qui est social est primordialement biologique. La méconnaissance de ce simple facteur est probablement suffisante à elle seule pour condamner tout le discours de la science sociale à l'ethnocentrisme des chrétiens. Car seule, à ma connaissance, l'évocation de la prégnance de la vision théologique infusée par Hobbes-Rousseau dans le mythe du « contrat social » nous permet de comprendre tant soit peu l'extraordinaire entêtement de la pensée occidentale à continuer de nos jours de refuser cette évidence : tout ce qui est social est biologique.

La vie sociale est un mixte à composition variable de compétition et de collaboration. La compétition demeure la règle de base de l'évolution et de la biologie mais elle se mâtine maintenant, dans la vie sociale, d'une collaboration qui est avantageuse d'une façon ou d'une autre dans la reproduction. Lorsque le biologiste se trouve en face d'une espèce sociale, il suppose que des facteurs environnementaux, dans le passé, ont favorisé une forme sociale de reproduction aux dépens de la reproduction de l'organisme solitaire. Le processus n'est pas irréversible, d'autres facteurs peuvent produire ou avoir produit l'effet inverse. On notera que cette supposition du biologiste n'est qu'une hypothèse au sens de la philosophie (supposition que l'on fait, vraie ou fausse, dont on tire des conséquences), et non pas au sens de la science, car elle n'a jamais été testée et démontrée. Une telle démonstration serait difficile, requérant possiblement la socialisation dans des conditions de laboratoire d'une espèce qui aurait été jusquelà solitaire. Cette carence est certainement une faiblesse de la biologie sociale actuelle comme science. Mais cette supposition de l'origine biologique du social est tout de même la seule qui soit raisonnable à l'heure actuelle. Malgré le titre de l'ouvrage de Darwin personne n'a jamais observé, non plus, la naissance d'une espèce.

Donc, le compromis qu'est la vie sociale est avantageux dans la reproduction des gènes d'une espèce, et cet avantage "se paye », s'obtient à un coût qui se traduit par une réduction de l'autonomie de l'organisme individuel. Par exemple, une abeille solitaire se reproduit elle-même ; lorsqu'une espèce de la famille des apidés devient sociale, l'organisme individuel de ces hyménoptères perd habituellement la capacité de se reproduire lui-même qui devient le monopole de la reine. Ainsi, et de multiples façons, la vie sociale implique des contraintes.

On a vu plus haut que chez les humains la morale occasionne une série de réductions dans l'exercice de la liberté. Certaines de ces contraintes morales ne concernent que le vivant individuel (elles existeraient même si nous n'étions pas sociaux) comme l'obligation de nourrir et protéger nos enfants. Mais on doit constater maintenant que dans la vie en général, et donc avant tout jugement moral, la vie sociale impose toute une série de contraintes spécifiques au comportement du vivant. De même que toute forme de vie sociale est un mixte de compétition et de collaboration, la vie humaine, qui est aussi sociale, est aussi un 
mixte de compétition et de collaboration. Il faut donc s'attendre à trouver dans la morale humaine les deux genres de contraintes, individuelles et sociales. C'est cet ensemble que j'appellerai les contraintes primaires qui, dans leur dimension proprement sociale, peuvent impliquer l'un ou plusieurs des domaines énumérés dans la liste non exhaustive suivante

\section{Quelques contraintes primaires sociales}

1 la division sociale du travail ;

2 la hiérarchie sociale, stratification sociale avec formation de castes ou classes ;

3 la dominance sexuelle, la répartition des rôles selon les sexes, la sélection sexuelle ;

4 les modes spécifiques de reproduction : monogamie, ou l'une ou l'autre des nombreuses formes de polygamie (polygynie ou polyandrie), monopole de la reproduction par un ou quelques organismes du groupe (les autres étant alors morphologiquement ou fonctionnellement stériles) ;

5 la dominance dans le groupe (et parfois entre groupes), qui procure un accès privilégié aux ressources ;

6 l'altruisme biologique : (= auto-sacrifice d'un organisme, qui a pour effet de réduire son aptitude à se reproduire); à la dominance des uns peut correspondre l'altruisme des autres ;

7 la symbiose sociale : commensalisme, mutualisme, parasitisme ;

8 la défense commune du territoire ou du nid, qui peut impliquer l'un ou plusieurs des autres facteurs mentionnés et l'ostracisme ou la xénophobie à l'égard de l'étranger au groupe ;

9. l'agressivité, considérée comme variable dépendante de la densité de la population ;

10. l'égalité des membres du groupe, la règle générale étant que plus une société est complexe, plus elle tend à être égalitaire.

Nous sommes des primates. Il existe environ deux cents espèces de primates (le nombre varie selon les experts). Certains primates sont arboricoles, d'autres sont surtout terrestres; certains sont minuscules, d'autres énormes comme le gorille dont le mâle peut peser jusqu'à deux cents kilos; sur deux cents espèces de primates, trente-sept sont monogames, soit dix-huit pour cent. Mais un seul 
primate, homo sapiens, est doté de langage et de morale. C'est ce que nous devons tenter de comprendre.

Le problème de l'origine de la morale est maintenant le suivant. Parce que nous sommes des vivants nous subissons l'effet de contraintes primaires, sociales et non sociales, qui sont autant de limitations à la liberté biologique. Parce que, d'autre part, nous sommes aussi des êtres humains nous subissons également les contraintes morales mentionnées précédemment et que nous avons dites observables et universelles chez tous les humains. Quelles relations peut-il y avoir, chez les humains, entre ces contraintes primaires applicables à tous les vivants et les contraintes morales qui sont propres aux humains ? Les effets produits par les unes et les autres étant en grande partie les mêmes, je propose de considérer que la morale humaine, qui est une série de limitations à la liberté biologique, est composée de l'ensemble des contraintes primaires. Les contraintes que nous appelons primaires, chez les autres animaux, nous les appelons des contraintes morales quand il s'agit d'êtres humains. On peut le voir en réexaminant de ce point de vue les trois exemples de contraintes morales cités plus haut : premier contact physique des parents avec le nouveau-né, évitement de l'inceste, altruisme.

1. Au premier contact physique avec son nouveau-né un être humain éprouve habituellement l'obligation de le nourrir et de le protéger. Il existe en biologie ce qui a été appelé "la stratégie du gros bébé ». La reproduction est un investissement de ressources, d'énergie et de temps. Certaines espèces répartissent cet investissement sur un très grand nombre de petits; la morue femelle, par exemple, peut pondre jusqu'à six millions d'œufs par saison de ponte, dont un grand nombre servira de nourriture à divers organismes marins, les survivants constituant la génération suivante de morues : c'est la stratégie dite du « petit bébé » dans laquelle le soin parental est réduit au minimum. D'autres espèces, au contraire, notamment chez les oiseaux, concentrent leur investissement sur un seul ou quelques rejetons dont ils s'occupent activement. On dit alors qu'il s'agit de la stratégie du "gros bébé ». Cette dernière est particulièrement visible chez les mammifères, et davantage encore chez les humains dont la période de maturation est exceptionnellement longue. La contrainte qu'éprouve l'être humain face à son nouveau-né et qu'il ressent comme contrainte morale est très probablement d'origine biologique et hautement fonctionnelle, car il est certain qu'un primate humain qui ne ferait pas l'investissement du soin parental réduirait considérablement les possibilités que ses gènes soient présents dans la génération suivante.

Deux remarques d'ordre général s'imposent ici avant de passer à l'examen du second exemple. Première remarque générale. L'exemple choisi, celui du nouveauné, est facile à voir et évident. Il existe par contre un très grand nombre de comportements humains qu'il serait beaucoup plus difficile d'expliquer en termes de biologie: la consommation de tabac, d'alcool et de drogues; l'infanticide, l'agressivité, le suicide, et bien d'autres. Que devrions-nous faire ? Devrions-nous 
vraiment tenter de comprendre et expliquer toute action humaine en termes de biologie ? La réponse est oui, même si l'on peut aisément concevoir que certaines questions devront probablement demeurer pour longtemps et peut-être pour toujours sans réponse. Tout exemple de comportement en apparence contreproductif ou à première vue biologiquement incompréhensible ne prouve rien à l'encontre d'une approche biologique, bien au contraire, un tel exemple milite en faveur d'une approche plus systématique mais expérimentale, par opposition à l'approche intuitive adoptée ici : une science. C'est précisément là où l'intuition nous laisse en panne qu'une approche expérimentale est le plus souhaitable et utile. Je suis conscient que cette façon de voir, qui sera développée dans le chapitre 2, semble aller à l'encontre du sens commun. Mais le sens commun, cette fois, est obscurantiste et ethnocentriste.

Deuxième remarque générale. Le raisonnement proposé plus haut dans l'exemple du nouveau-né n'est pas assimilable à un réductivisme sans limite qui dirait en substance : " ceci n'est que cela ». Il s'agit au contraire de mettre en rapport et de comparer des phénomènes appartenant à deux sphères de connaissances que l'on a l'habitude de considérer comme inconciliables et irréductibles. D'une part, la sélection naturelle qui est le moteur de la théorie de l'évolution des vivants et d'autre part, quelques phénomènes observables et universels appartenant à la dimension morale de la vie humaine. Il s'agit de considérer que la morale humaine ne tombe pas du ciel et ne naît pas des bonnes intentions ; au moins dans ce qu'elle a de plus universellement observable, nous devons adopter la perspective qui en fait une partie intégrée dans un tout qu'elle prolonge et consomme.

2. L'inceste. En général, les animaux ne pratiquent pas l'inceste. Il y a des exceptions, comme l'accouplement frère-sœur chez quelques insectes. La question générale de l'inceste est compliquée et je me limiterai au rappel d'une des composantes biologiques résumées par Wilson ${ }^{1}$. D'une part, l'inbreeding inhérent au petit groupe favorise l'altruisme entre organismes apparentés multipliant les gènes qu'ils ont en commun. Par contre, le même inbreeding abaisse l'hétérozygosité, ce qui peut mettre en péril l'existence du groupe par l'affaiblissement de l'adaptabilité génétique. Le biologiste suppose alors que l'organisation de la vie sociale du groupe se situe probablement au point d'équilibration de ces deux forces opposées. De ce problème complexe que la science actuelle n'a pas encore résolu dans tous ses détails contentons-nous de retenir l'existence, chez les organismes sociaux, d'importants facteurs d'autolimitation de l'inceste. Ce qui est suffisant pour nous amener a penser que l'interdit de l'inceste des humains prolonge, au moins en partie, des contraintes présentes chez les animaux.

Edward O. Wilson, Sociobiology, p. 79-80 ; Lumsden et Wilson, 1981, p. 86. Voir également Michael Ruse, Taking Darwin seriously, p. 145-147. 
Chez les humains, des études du développement de l'enfance menées en Israël et à Taiwan montrent que l'inhibition à l'égard de l'inceste apparaît à l'approche de la nubilité entre garçons et filles élevés ensemble comme frères et soeurs lors même qu'ils ne sont pas biologiquement apparentés.

John Maynard Smith reconnaît pour sa part que la biologie ne peut rendre compte à elle seule des différences appréciables existant entre les diverses formes culturellement différentes de l'interdit de l'inceste. Mais il ajoute, et nous suivrons cet avis :

Les différences existant dans les coutumes des diverses sociétés humaines demandent une interprétation culturelle qui a peu à voir avec l'aptitude intégrée biologique. Mais celui qui voudrait présenter une explication générale des coutumes du mariage humain et des tabous de l'inceste en méconnaissant le fait que, selon toute probabilité, longtemps avant d'être dotés de langage nos ancêtres évitaient déjà de s'accoupler avec leurs proches parents, celui-là ferait montre d'un esprit stupidement chauvin [foolishly parochial ${ }^{1}$.

Là encore, la règle morale de l'évitement de l'inceste chez les humains semble prolonger l'absence de conduite incestueuse chez les animaux. L'interdit de l'inceste ne constitue certainement pas une invention proprement humaine, l'acte fondateur par lequel nous passerions de la nature à la culture comme l'affirme Claude Lévi-Strauss ${ }^{2}$. Quant à la version freudienne de l'interdit de l'inceste contre laquelle Lévi-Strauss a si vaillamment polémiqué, là où le psychanalyste tente de nous convaincre que le mâle humain désire fortement copuler avec sa mère, le biologiste se borne à constater que le mâle humain désire fortement copuler.

3. L'altruisme. Lorsque, de propos délibéré, nous sommes altruistes au sens moral du terme, nous appliquons une règle dont l'effet est d'avantager quelqu'un à nos dépens, généralement un proche. Lorsqu'un organisme non humain est altruiste, il applique une règle, qui est une des premières qui aient été identifiées en biologie sociale et dont l'effet est d'accroître la probabilité de reproduction d'un autre organisme, aux dépens de ses propres chances de reproduction.

L'intérêt exceptionnel de la théorie génétique de l'altruisme est particulièrement visible dans sa première formulation par William D. Hamilton en $1964^{3}$, qui démontre que l'altruisme peut être produit par sélection naturelle ou par une forme quelconque d'évolution, et cela non pas en identifiant un gène particulier qui occasionnerait un comportement altruiste du phénotype mais plutôt comme simple résultat d'une singulière distribution des gamètes dans l'espèce. Chez les hyménoptères étudiés par Hamilton, les mâles sont haploïdes (leurs chromosomes sont simples) et n'ont pas de père génétique, la totalité de leur matériel génétique

John Maynard Smith, The Evolution of Sex, p. 145.

Lévi-Strauss, Claude (1967). Les Structures élémentaires de la parenté, p. 28-29.

Hamilton, William D. (1964). « The Genetic Evolution of Social Behaviour ». 
provenant de leur mère ; alors que les femelles sont diploïdes (leurs chromosomes sont doubles), elles héritent du matériel génétique du père et de la mère et sont de ce fait génétiquement plus proches de leurs sœurs (3/4) que de leur mère (1/2). L'altruisme des hyménoptères et la stérilité de leurs castes ouvrières, problème qui avait hanté Darwin au point de lui faire dire que toute sa théorie de la sélection naturelle dépendait de sa solution, résulte de ce fait. Le concept central d'Hamilton d'aptitude intégrée (inclusive fitness) nous fait comprendre que l'altruisme biologique a pu apparaître en l'absence de tout gène particulier occasionnant à lui seul un comportement altruiste, même en l'absence de l'espèce humaine.

\section{La morale n'est pas composée uniquement de choses que notre ethnocentrisme se plaît à regarder}

Je résume. La liberté biologique rend possibles un certain nombre de performances physiques. Des contraintes primaires, appelées contraintes morales dans le cas des êtres humains, viennent limiter le nombre et l'exercice de ces performances.

Il existe cependant des contraintes primaires dont nous pouvons trouver des traces observables et universelles dans le comportement humain et que, néanmoins, nous refusons d'ordinaire d'inclure au nombre des contraintes de la morale humaine. Je veux parler principalement de la xénophobie, du sexisme (tout comportement ayant pour effet l'infériorisation sociale de la femme), et de l'inégalité. La xénophobie sera l'objet d'une étude particulière au chapitre 4 et ne sera qu'esquissée ici.

Nous faisons face au point le plus important et à la difficulté la plus cruciale pour notre compréhension de la morale humaine. Lorsque, plus haut, j'ai défini la morale comme une série de contraintes, je n'ai pas dit que cette série est composée uniquement de contraintes déjà reconnues et acceptées, comme l'obligation de prodiguer le soin parental, l'interdit de l'inceste ou l'altruisme, autrement dit composée uniquement de conduites qui nous plaisent et qui sont reconnues dans le code moral ou la conscience morale ou les chartes des droits que les sociétés humaines revendiquent et proclament. J'ai dit que la morale est faite de contraintes. Il est de la plus haute importance de ne pas changer le contenu d'une définition en cours d'exposé, sur ce point ou sur tout autre. Si donc la morale est une série de contraintes, et si la xénophobie (et ses diverses formes comme l'ostracisme, l'ethnocentrisme et le racisme) est, comme je le soutiendrai dans les pages qui suivent, universellement observable dans le comportement humain et au nombre des contraintes biologiques primaires, alors il faut dire que la xénophobie appartient à la morale humaine. Je ne vois pas comment on pourrait échapper à cette conclusion. Je sais que la reconnaissance explicite de ce fait semble aller à l'encontre de tout ce que la morale représente traditionnellement en Occident mais je maintiens qu'une compréhension articulée et descriptive de ce qu'est la morale humaine est à ce prix. Si la morale est faite de contraintes primaires et si la 
xénophobie est une contrainte primaire, alors la xénophobie fait partie de la morale. La xénophobie appartient à la nature humaine.

Or, il existe bel et bien un principe de xénophobie en biologie sociale. On l'appelle le «principe » parce qu'il a été vérifié et documenté dans pratiquement tous les groupes d'animaux vivant en société possédant une vie sociale un peu complexe et qui ont été examinés sous ce rapport. Sans être universelle, la xénophobie animale est très fréquente dans les sociétés animales ${ }^{1}$. Chez les lions, les fourmis ou les primates, par exemple, l'apparition d'un étranger constitue le plus fort stimulus de conduites agressives dont les membres sont capables et qui sont alors dirigées contre l'intrus. La présence de l'étranger représente une menace au statut social, au rang de chacun des membres du groupe, elle provoque les plus grands désordres qui ne cessent qu'avec son éviction, parfois sa mort ou éventuellement son intégration après une période de probation plus ou moins longue. La généralité de la xénophobie dans les sociétés animales nous suggère l'idée que sa présence est probablement sociogène, qu'elle facilite la vie sociale dont elle renforce la cohésion et la stabilité.

La difficulté que nous éprouvons ici provient principalement du fait que la morale humaine est dite, et que la morale occidentale moderne ne dit pas qu'elle est xénophobe. La morale est exprimée, exposée dans un discours moral, un code, des lois, des principes, des chartes, des catéchismes et notre société croit, et avec elle chaque société humaine, que sa morale n'est que ce qu'elle dit qu'elle est. En réalité toutes les sociétés humaines sont ethnocentristes, chacune croit à la supériorité inhérente de sa propre culture et croit que sa morale n'est que ce qu'elle dit qu'elle est, telle qu'elle se la représente dans son discours particulier.

Il faut donc reconnaître que dans l'ensemble des contraintes primaires qui constituent la morale, toute société humaine opère un choix, une sélection qu'elle se représente dans son discours explicite, dans ses pratiques collectives et dans ses institutions. Le primate humain est un primate parlant, il est le seul dont le système nerveux est (de quelque façon sans importance pour notre propos), à la source de ce que nous appelons la conscience de soi et le langage. Lorsque le primate parlant se représente à lui-même ce qu'il est, l'ensemble des contraintes qu'il revendique pour lui-même et qu'il reconnaît explicitement comme autant de principes de sa vie morale n'est qu'un sous-ensemble des contraintes primaires qu'il subit comme organisme et auxquelles son action est astreinte.

Il convient de rendre plus explicite le rôle assigné ici au langage et à la conscience. À cette fin, on peut avoir recours à l'image évoquée par Gregory Bateson ${ }^{2}$ de l'impossibilité de construire un téléviseur dont l'écran ferait voir

1 Voir Johan M. G. van der Dennen, « Ethnocentrism and In-group/Out-group Differentiation : a Review and Interpretation of the Literature », p. 20.

2 Bateson, Gregory (1977). Vers une écologie de l'esprit, I, note de la page 148. 
toutes les parties qui le composent; tel est le rôle assigné ici au discours et à la conscience que nous avons de ce que nous sommes. Cette image ne tient évidemment pas lieu d'une théorie du langage ou de la conscience, mais veut seulement laisser entendre que, de même qu'un tel appareil ne saurait exhiber la totalité de ce qu'il est, la conscience que nous avons de notre existence morale ne peut être ou contenir qu'un sous-ensemble d'un ensemble plus vaste. La présente explicitation, ou le recours à l'image de Bateson, n'a donc pas de valeur en soi et ne prétend pas définir la nature même du langage ; elle est seulement descriptive d'un usage et n'est proposée que pour des raisons de commodité et de clarté de l'exposé.

\section{Les contraintes secondaires}

J'appellerai contraintes secondaires les contraintes appartenant au sous-ensemble de la morale dite.

Les contraintes primaires sont biologiques, elles sont produites par l'évolution darwinienne, elles sont les mêmes pour toute l'humanité et elles opèrent toujours, qu'elles soient dites tacites ou au contraire déniées. Les contraintes secondaires, de leur côté, varient selon les sociétés et les cultures. Pour toute société humaine donnée ces contraintes secondaires forment un sous-ensemble des contraintes primaires ; parce qu'elles sont dites et intégrées à la culture d'une société elles sont cette partie de notre morale qui apparaît à notre conscience et que nous revendiquons : ces contraintes secondaires sont nos « valeurs ». Leur évolution est moins darwinienne que lamarckienne par la transmission culturelle, d'une génération à l'autre, des discours et des traditions moraux. Chaque ensemble de contraintes secondaires est particulier à l'histoire culturelle de la société qui l'applique. Les contraintes secondaires ne sont effectives et leurs effets ne sont observables que dans la mesure où elles renforcent ou appliquent des contraintes primaires, et qu'elles ne vont pas à l'encontre d'autres contraintes primaires. Le discours moral qui nous rappelle l'obligation de nourrir et protéger nos enfants est très suivi, celui qui nous enjoint de ne pas être raciste l'est beaucoup moins ; la contrainte primaire est toujours pourvue d'effets, la contrainte secondaire ne l'est qu'à certaines conditions seulement.

Dans l'histoire, la morale chrétienne qui se représente volontiers à elle-même dans l'universalité du "Aimez-vous les uns les autres» du Sermon sur la Montagne s'est longtemps matérialisée dans la singularité de son Inquisition et s'est accompagnée de la xénophobie longtemps féroce de son antisémitisme. Le fait que la morale chrétienne actuelle ne reconnaît pas explicitement l'origine biologique des contraintes secondaires qu'elle met en œuvre et qui, par rapport à l'ensemble des contraintes biologiques, ne constituent qu'un sous-ensemble culturel et sélectif, ne l'empêche nullement de les appliquer, il ne fait que lui interdire de le savoir et de le penser. L'un n'empêche pas l'autre, bien au contraire, l'un passe par l'autre. La morale chrétienne, comme tout autre morale dite, est ethnocentriste, elle s'interdit de penser qu'elle pourrait être autre chose que ce qu'elle pense qu'elle est, 
en conséquence de quoi sa propre xénophobie n'est pas du tout rendue irréelle mais seulement impensable. Sa méconnaissance de ce qu'elle est vient confirmer son incapacité de penser cela qu'elle a rendu impensable pour elle-même. " On ne juge pas un homme sur l'idée qu'il a de lui-même », disait Karl Marx. On ne jugera pas davantage une morale dite sur l'idée qu'elle présente d'elle-même, mais plutôt sur ce qu'elle fait et sur les effets observables qu'elle produit. Si elle produit des effets xénophobes et qu'elle affirme néanmoins n'être pas xénophobe, nous dirons qu'elle est xénophobe et dit qu'elle ne l'est pas. Où est le problème ?

Je pense que sur ce point il n'y en a plus. Nous avons toutes les raisons de penser que la xénophobie et le racisme font partie de la nature humaine depuis très longtemps. Au point que l'apparition des termes eux-mêmes de xénophobie et racisme semble incroyablement récente. Selon Le Petit Robert, « xénophobe » date du début du $X^{\mathrm{e}}$ siècle, " raciste » apparaît vers 1930. Ce qui signifie qu'avant le $\mathrm{XX}^{\mathrm{e}}$ siècle et probablement depuis la nuit des temps les sociétés humaines ont toujours été racistes. Il en va de même en anglais. L'apparition relativement récente du mot n'indiquerait alors que le moment où certaines de ces sociétés racistes ont cessé d'être fières de l'être.

La morale humaine est faite de contraintes primaires d'origine biologique réalisées par le truchement des contraintes secondaires. La morale dite est donc faite de valeurs, dorénavant et tel que promis dans l'introduction je ne mettrai plus de guillemets au mot valeur ; elle est un sous-ensemble culturellement sélectif de contraintes secondaires réalisant les contraintes primaires. Alors que les contraintes primaires résultent d'une évolution darwinienne, les contraintes secondaires de la morale dite sont soumises à une évolution de type lamarckien, transmises par voie culturelle qui est potentiellement additive ou cumulative.

\section{L'immoralité}

Il est maintenant possible d'assigner un sens objectif au terme « immoralité ». L'action qu'on appelle immorale est soit l'effet de contraintes primaires qui n'ont pas été confirmées au niveau secondaire, soit l'effet de contraintes secondaires allocentriques, c'est-à-dire qui appartiennent à un autre ethnocentrisme que celui de la société dans laquelle l'action prend forme. Par exemple la polygamie musulmane qui serait pratiquée en Occident ou la consommation publique d'alcool en pays de tradition musulmane.

L'immoralité voyage mal. Étant donné qu'il n'existe pas de société humaine sans contraintes secondaires, sans morale dite, et étant donné qu'aucune société humaine ne détient la formule idéale des contraintes secondaires ou de monopole de la vérité nonobstant le fait que chacune y prétende à l'occasion, il s'ensuit que toute affirmation concernant l'immoralité d'une action n'a de sens qu'à l'intérieur de la société dans laquelle un telle imputation est faite, en dehors de laquelle cette affirmation n'est que l'indice de son ethnocentrisme. 
Quand l'immoralité est en jeu nous n'avons guère de choix. Il serait bon de se rappeler que les anciens Grecs avaient un mot pour dire " choix », hairesis, dont nous avons fait " hérésie ». Pour le groupe, choisir c'est toujours être hérétique. Celui qui choisit est un traître. Traditionnellement, dire qu'une action est immorale n'est que la première étape d'un processus d'expulsion.

L'immoralité n'est pas un sujet simple. Elle se situe dans une sorte de «zone grise », pour ainsi dire, entre les contraintes primaires et secondaires, elle est constituée d'actions qui sont parfois considérées comme immorales et parfois non pour diverses raisons. La première étant que, pour le langage, cette région est instable ou changeante, elle comporte fréquemment des actions humaines dont certaines sont sur le point de cesser d'appartenir à l'immoralité, alors que d'autres performances sont au contraire en voie d'y entrer. Exemples : en Occident à l'heure actuelle, sur la voie de sortie de la zone d'immoralité on trouve les manquements commis au sens de l'honneur, les relations sexuelles avant le mariage, l'avortement et le divorce ; sur la voie d'entrée on trouve les " crimes contre l'humanité », le harcèlement sexuel, la violation des «droits des animaux », le racisme. Et ce n'est pas tout, la zone grise est aussi alimentée par la vaste problématique des mœurs, c'est-à-dire toute cette partie de notre conduite pour laquelle nous ne reconnaissons qu'avec une certaine répugnance la part qu'elles peuvent occuper dans notre morale, part dont nous ne sommes qu'à peine conscients ou que nous éprouvons les plus grandes difficultés à intégrer de plein droit dans notre conception explicite de la vie morale. Par exemple, un musulman pourrait se croire justifié de nier vigoureusement que la clitoridectomie soit véritablement une coutume musulmane. À strictement parler, du point de vue de la morale musulmane en tant que morale dite, explicite, ce point de vue semble justifié puisque le prophète Mohammed n'a pas prescrit cette pratique dans le Coran. Mais la coutume en est si largement répandue en Afrique et ailleurs où l'Islam (avec d'autres religions) est présente qu'il faut bien, d'une façon ou d'une autre, se poser des questions et s'interroger sur l'existence des responsabilités sur ce phénomène aux proportions impressionnantes. L'édition 1982 du Hosken Report ${ }^{1}$ dénombrait 84 millions de femmes, la plupart musulmanes, ayant subi la clitoridectomie ou l'infibulation, ou les deux. Un problème analogue se pose à l'Église catholique qui refuse aux femmes l'accès à la prêtrise et à la hiérarchie de l'administration religieuse, alors que Jésus n'a évidemment jamais autorisé ce genre de discrimination. Autant de raisons qui nous feront revenir sur la question de l'immoralité dans le chapitre 2.

Je répéterai que ces considérations ne relèvent pas d'un « relativisme moral » ou d'une incompréhension devant la diversité des versions de la morale humaine et qui se donnerait des airs de libéralisme ou de largesse d'esprit. Aucun être humain ne peut être moralement relativiste si ce n'est par un pur verbalisme sans

Fran Hosken (1982), The Hosken Report : Genital and Sexual Mutilation of Females. Selon une évaluation plus récente (Scientific American, août 1994, p. 83) ces mutilations seraient infligées en moyenne chaque année à deux millions de fillettes vivant dans vingt-six pays africains. 
conséquence. Chacun appartient à une société qui médiatise d'une façon particulière les contraintes secondaires qui font de lui un être moral d'un lieu et d'une époque.

\section{Tableau 2}

Rapports entre contraintes primaires, contraintes secondaires et immoralité.

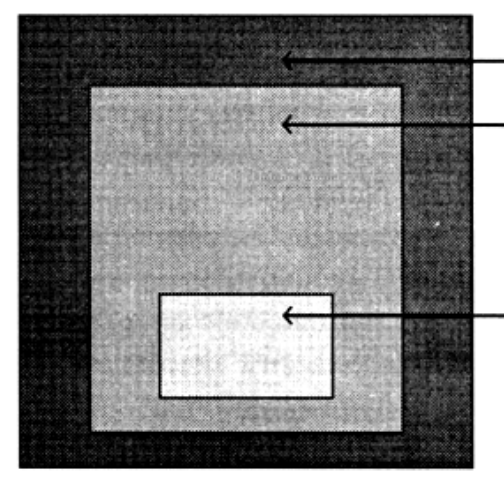

Vie. Liberté.

Contraintes primaires

(animaux).

Morale (humains), incluant l'immoralité.

Contraintes secondaires.

Morale dite, éthique

ou valeurs, excluant

l'immoralité.

On ne peut s'empêcher de remarquer la relative inefficacité des contraintes secondaires par lesquelles les sociétés tentent de réduire l'effet des contraintes primaires. Par exemple, il semble que le primate humain soit modérément polygame; la monogamie longtemps tenue pour être la seule forme morale de l'union conjugale en Occident s'accompagne depuis toujours, semble-t-il, de ce qui a été appelé la "monogamie à répétition », "l'éternel triangle », le " divorce à l'italienne », etc. Je préférerai, pour ma part, m'en tenir à des formules moins cyniques et plus descriptives, comme celle qui a été utilisée plus haut: la contrainte primaire est toujours suivie d'effet, la contrainte secondaire ne l'est qu'à certaines conditions. Toute société humaine fait face à ce genre de problème qui est celui de la relative inadéquation des contraintes secondaires qu'elle se donne par rapport aux contraintes primaires, chacune le résout d'une manière qui lui est propre, mais l'approche descriptive nous permet de penser que cette relative inadéquation, qui est une donnée permanente de la vie sociale humaine, la rend aussi indéfiniment perfectible. L'approche cynique, au contraire, théâtralise l'incapacité de l'approche individualiste et subjectiviste à rendre compte objectivement de la morale, elle tente de nous faire rire pour nous mieux persuader d'oublier sa stérilité et de la sorte nous y mieux maintenir. Le cynique appartient fréquemment à ce qu'il dénonce, spiritualiste malheureux réfugié dans l'amer plaisir de vitupérer l'époque. Selon l'étymologie le point de vue cynique est celui des chiens. Nous le leur laisserons. 
L'approche descriptive a deux avantages. Le premier est de nous tenir informés de la relative inadéquation des contraintes secondaires que nous adoptons. Lorsqu'une contrainte secondaire, comme la monogamie en Occident ou l'interdiction de l'alcool en pays d'Islam, est régulièrement enfreinte sur une longue période de temps par une fraction importante de la population, cela peut nous instruire, soit de la puissance jusque-là insoupçonnée d'une contrainte primaire qui prévaut malgré toutes les précautions qu'une société prend contre elle, soit de l'existence de privilèges sociaux détenus par une fraction de la population, ou d'autres choses encore.

Le second avantage des formules descriptives et non cyniques est plus grand encore, qui est de nous rappeler le rôle social de la morale. Dans une société humaine, la morale, interprétée d'un point de vue fonctionnel, joue un rôle qu'on pourrait assimiler à un "système immunitaire » du groupe. Un organisme vivant est composé d'un grand nombre de cellules et se dote ordinairement d'un système immunitaire dont la fonction est d'offrir une résistance à l'invasion des corps étrangers susceptibles de mettre en péril son unité structurale et fonctionnelle. Si l'on accepte, pour les besoins de la présente discussion, de considérer une société humaine comme une totalité vivante, on peut voir que la morale dite que se donne un groupe humain, l'ensemble de ses contraintes secondaires, joue le rôle d'une puissante force centripète contribuant à la cohésion et à la stabilité sociales et permet à cette société de contraindre et éventuellement d'isoler ou de chasser les auteurs de comportements qui s'en écartent comme s'ils étaient autant de corps étrangers.

Enfin nous pouvons réexaminer maintenant la question posée dans l'introduction sur l'ethnocentrisme et sur l'existence possible de sciences sociales qui seraient moralement neutres et value-free : de telles sciences sociales sont-elles possibles?

Se poser cette question revient à se demander ce qu'est une valeur. Il est maintenant devenu évident que ce que nous appelons une valeur est l'appellation ethnocentriste que la société occidentale attache aux contraintes secondaires qu'elle s'est données, qu'elle énonce sur le mode absolu typique des droits de la personne et qui n'ont d'effet que relativement aux contraintes primaires qu'elles appliquent.

S'il est vrai que toute forme de vie sociale est un compromis créé par l'évolution de la vie et dont l'effet est, par le truchement de contraintes diverses, d'abaisser l'âpreté de la compétition décrite par Darwin, il est en vérité douteux que les sciences sociales, si elles tiennent à demeurer descriptives, puissent jamais se libérer complètement des valeurs. On ne peut pas imaginer de société animale sans contrainte primaire ni une société humaine sans contraintes secondaires, et donc sans valeurs. Étant donné que les contraintes secondaires sont généralement spécifiques à chaque groupe, on doit s'attendre à ce que chacun des groupes 
différents colore différemment son discours de ses tendances et préventions particulières.

Ce qui ne revient pas à dire que la chose soit impossible. Une telle affirmation serait hors de ma portée ; comme dit Nicholas Rescher : " Aucun problème décrit par le discours cohérent ne peut vraiment être dit hors de portée de la science ${ }^{1}$. Mais nous n'avons nul besoin d'une généralisation de ce genre car le fait qu'il importe de garder à l'esprit et dont il faudrait tenter de rendre compte, c'est l'échec historique des sciences sociales à réaliser l'idéal qu'elles se sont donné.

Nicholas Rescher, The Limits of Science, p. 132. 


\section{Chapitre 2}

\section{Plaidoyer pour une morale expérimentale}

Natura non nisi parendo vincitur

Francis BACON

\section{$\underline{\text { Retour à la table des matières }}$}

Je suggère la création d'une science morale expérimentale et appliquée, formée sur le modèle de cette autre science expérimentale et appliquée qu'est la médecine.

Les résultats auxquels nous sommes parvenus jusqu'ici nous permettent une certaine compréhension et une interprétation de l'échec de la science positive, ou d'inspiration positiviste, depuis plus d'un siècle, à se doter de sciences sociales et humaines non moralisatrices et non entachées de valeur. L'ethnocentrisme de la science sociale est apparemment indépassable. Pour en comprendre les raisons il faut comprendre ce que c'est qu'une valeur et de quoi elle est faite. Si ce que nous désignons encore confusément par le terme de valeur est en réalité une contrainte, il devient possible de prendre acte de cet échec relatif et d'en tirer leçon. Cette leçon, en fait, est aussi ancienne que la science elle-même : "Natura non nisi parendo vincitur », disait Bacon dans son Novum Organon, on ne lutte contre la nature qu'avec son aide. C'est aussi vrai de nos jours qu'en 1620. Pour lutter contre la nature il faut d'abord lui obéir, il faut la comprendre et identifier les régularités dont elle est faite si l'on veut être en mesure de les tourner à notre avantage. La morale humaine est une telle régularité. On n'abolit pas une régularité de la nature, dans le cas de la morale comme dans celui de la gravitation, cela n'est pas en notre pouvoir. Ce qui est en notre pouvoir, toute l'histoire de la science le montre, c'est la progressive réappropriation, à des fins humaines, des régularités observables. La gravitation n'empêche pas, c'est elle seule au contraire qui permet la construction des centrales hydroélectriques et le vol des avions. Même Kant savait en son temps 
que la résistance de l'air rend possible le vol de la colombe. Il pourrait en être de même de la morale qui est une contrainte imposée par la vie. La vie c'est la liberté et c'est l'improbable dont nous pouvons apprendre à nous servir à des fins que nous pouvons nous donner et qui nous sont propres.

À l'intention du philosophe qui se désole du désert moral, du gouffre qui se creuse et va croissant de nos jours entre la science et le reste de la culture, on peut ajouter une variation moderne à l'apophtegme de Bacon: Scientia non nisi parendo vincitur. C'est à la science et à l'expérimentation qu'il faut demander de nous instruire de quoi est faite notre morale qui fait échec à la science sociale depuis un siècle, afin de mieux mettre ce savoir au service de l'humanité.

\section{La médecine est anti-eugéniste}

Une science expérimentale de la morale devrait prendre pour modèle la médecine. Depuis ses tout débuts la médecine lutte contre certaines conséquences indésirables de l'évolution. Pour le comprendre, nous devons à nouveau retourner à Darwin. La vie est une compétition qui assure la postérité du plus apte. La permanence de cette compétition est une « loi de la nature » dans le sens que, tout comme la gravitation, aucune force connue et certainement aucune entreprise humaine ne saurait l'abolir, elle ne peut qu'en déplacer les effets. Comme on l'a vu l'évolution se charge elle-même de déplacer certains effets de cette compétition et par là d'en réduire l'impact au moyen de divers compromis qui, comme la vie sociale, contribuent à produire ce que Darwin a appelé les « animaux supérieurs ». Autrement dit, l'évolution est eugéniste: elle élimine les formes de vie entre lesquelles l'intensité de la compétition quotidienne donne lieu à un taux de reproduction inférieur à celui que permettent d'autres formes de vie pour lesquelles l'âpreté de la compétition est moindre. L'évolution est opportuniste, aussi opportuniste que peut l'être un litre d'eau dans un vase poreux et dont chaque molécule trouve à se loger au point le plus bas que permet l'environnement constitué par le vase et les autres molécules.

La médecine, au contraire, est anti-eugéniste. Comme science appliquée et depuis ses débuts la médecine est au service des humains, elle soigne des infections, panse des blessures, répare des difformités congénitales qui, chez les autres primates, éliminent les organismes porteurs ou les rendent moins aptes à se reproduire. En ce sens la médecine est anti-évolutionniste, anti-eugéniste et humaniste. Longtemps déjà avant que l'humanisme ne devienne à la mode en Occident à partir de la Renaissance et à l'époque de Rabelais, la médecine était humaniste. Depuis Hippocrate qui vivait en Grèce il y a environ 2400 ans, elle conserve presque intact le même idéal : être utile à la vie humaine ou au moins ne pas lui nuire. Quand on la compare, comme pratique humaine, à toutes les traditions morales qui ont précédé la nôtre, à la plupart des religions connues et à presque tous les régimes politiques, il semble que la médecine n'ait pas, ou ait infiniment moins, autorisé ou simplement cautionné l'avilissement ou 
l'asservissement de la vie humaine à d'autres fins qu'elle-même. Sa règle fondamentale a toujours été d'être utile, ou au moins de ne pas nuire à la vie du corps. La médecine n'a même jamais prétendu empêcher les hommes de s'entretuer, se contentant de suivre les progrès de la science des armements ; Galien (né vers 131 de notre ère, mort vers 201), a commencé sa carrière en soignant des gladiateurs romains. Et il faut attendre les temps modernes pour voir sur une grande échelle l'inféodation politique de médecins surveillant les signes vitaux des prisonniers torturés en Allemagne et ailleurs, ou des victimes psychiatrisées des prisons communistes. Ou encore, plus près de nous, les expériences menées dans un institut psychiatrique de l'Université McGill à Montréal de 1950 à $1965^{1}$ subventionnées par le Gouvernement canadien et la Central Intelligence Agency (CIA). Il faut dire que cette relative innocuité historique de la médecine a longtemps été concomitante au relatif éloignement de ses praticiens des cercles du pouvoir politique, ce qui est moins vrai de nos jours.

La morale humaine, par contre, a une histoire infiniment plus variée. Certes, la morale occidentale actuelle est humaniste et en ce sens elle est aussi anti-eugéniste et anti-évolutionniste, mais cet avatar est récent et encore limité à une fraction de la population humaine. On demeure confondu, de nos jours, par l'ampleur de l'écart avalisé par la pratique morale de la plupart des sociétés d'avant le XVIII ${ }^{\mathrm{e}}$ siècle, qui séparait le statut social des nobles de celui du reste de la société ; l'importance des privilèges réservés aux despotes et aux familles princières, la dominance du mâle, le rôle de l'infanticide, les formes de l'intolérance religieuse. Et l'extraordinaire brutalité, pour des yeux modernes, des châtiments réservés aux criminels, aux déviants, aux prisonniers de guerre, aux « infidèles » de toute sorte. Jusqu'à Kant, l'idée même de morale est attachée à la naissance, au rang et à l'éducation, c'est un privilège de classe. Pour Aristote c'est la moralité humaine elle-même qui est inaccessible à ceux qui pratiquent certaines occupations viles et qui ne sont donc pas considérés comme des êtres humains de plein droit.

En d'autres mots, lorsqu'on examine l'histoire de la morale humaine pour ce qu'elle est, c'est-à-dire non pas dans les livres où les philosophes en parlent mais dans l'histoire des contraintes secondaires que le primate parlant s'est données et qui réalisaient des contraintes primaires, il est certain qu'aucun qualificatif ne saurait rendre justice à l'extrême variété des phénomènes qu'on y trouve, mais il est également évident que l'on n'a que trop souvent l'occasion de reconnaître à quel point cette histoire est en effet celle d'un primate. D'un primate qui, bien que sachant manifester à l'occasion une capacité d'entraide dont peu d'animaux sont capables (les exceptions, de faible importance, se trouvent chez le dauphin et l'éléphant), et de soins prodigués aux vieillards (pour lesquels je ne connais pas d'exemple dans le monde animal), il reste qu'à de nombreux égards l'histoire de la vie sociale du primate parlant est longtemps demeurée relativement proche, toute

Anne Collins, Anne. Les Patients du docteur Cameron : L'Étonnante histoire des expériences de lavage de cerveaux financées par la CIA dans un hôpital canadien. 
proportion gardée, des autres formes de vie sociale que l'évolution a réalisées chez les autres primates et les autres animaux.

La comparaison qui précède entre la médecine et la morale peut paraître injuste. D'abord parce que la médecine ne s'occupe que du soin du corps alors que la morale veut s'occuper de l'esprit et de l'«âme ». Et ensuite à cause de la vocation si différente de chacune. On peut en effet alléguer que, quelle que soit l'allégeance particulière de chacun des antagonistes d'un conflit, ils ont tous intérêt à favoriser et protéger la pratique de la médecine dont chacun peut profiter un jour ou l'autre, alors que la vocation de la morale d'un peuple est très différente et surtout infiniment plus vaste et se situe davantage au cœur même de l'existence humaine en ce qu'elle a de plus essentiel.

Mais ce genre de raisonnement constitue un rappel à l'ordre effectué par une forme particulière d'ethnocentrisme en matière morale et par sa prétention à une vocation universelle qu'aucune morale historique n'a jamais réalisée dans les faits. L'esclave de la Grèce classique n'était certainement pas considéré comme un être humain à part entière ou comme un citoyen, ni la femme dans l'empire romain et dans bien d'autres régimes, ni le musulman ou le Juif au Moyen Age, ni l'Africain esclave jusque vers 1850. Ce n'est pas la médecine qui a inventé les bûchers de l'Inquisition et ce n'est pas l'Église catholique qui a libéré l'humanité de la variole. Je continuerai à soutenir que si nous désirons commencer a apercevoir l'importance, la permanence, et à l'occasion la férocité de cet ethnocentrisme et d'en apprécier le rôle, il peut être fort instructif de remarquer qu'au cours de la plus grande partie de son histoire préscientifique la pratique de la médecine a été plus constamment humaniste que ne l'a été la pratique morale humaine au cours de la même période. En fait la médecine semble être un meilleur guide pour la constitution d'une science de la morale que toute autre discipline que l'on pourrait évoquer.

\section{La médecine comme science expérimentale}

On fixe habituellement la naissance de la médecine comme science expérimentale dans les travaux de Claude Bernard. Dans son Histoire de la médecine Charles Lichtenhaeler attribue une bonne part du mérite à François Magendie (1783-1855) dont Bernard fut le disciple.

L'intérêt de la médecine comme modèle d'une science expérimentale de la morale est multiple. En premier lieu, on a souvent remarqué que la science de la médecine a davantage progressé dans le passé par l'étude de la maladie que par celle de la santé. La médecine existe parce que, de nombreuses façons, les humains souffrent et que l'étude de leurs maux, plaies et afflictions nous renseigne davantage sur la composition et le fonctionnement du corps humain que ne le fait l'étude du corps en santé. On serait porté à dire que de même que les peuples heureux sont réputés sans histoire, de même les corps sains n'ont pas de médecine ; 
cela était vrai au siècle dernier, ce l'est moins de nos jours par la multiplication des études des conditions de la vie moderne. Mais en morale, tout au contraire, presque toute la tradition s'est attachée à définir le Bien exprimé sous forme d'une valeurqui-a-une-valeur, autrement dit d'un ethnocentrisme culturel, le Mal étant tout ce qui lui est opposé, généralement tout le reste, tout ce qui est à l'extérieur de luimême et ne lui ressemble pas. Il me semble qu'en se modelant sur l'histoire de la médecine l'étude de la morale pourrait adopter le point de vue que la morale nous importe parce qu'il existe, chez les humains, des problèmes dont certains sont particulièrement criants et intolérables, parce que nous sommes racistes par exemple, sexistes, intolérants, violents, injustes ou cruels. Une telle approche, interventionniste par principe comme peut l'être l'approche médicale qui met la science au service des gens au lieu de mettre les gens au service d'un pouvoir politico-religieux, procurerait un tout autre point de départ à la recherche et à la réflexion morales.

En second lieu, la médecine expérimentale est aussi intéressante comme modèle d'une science morale parce qu'elle s'efforce de reproduire en laboratoire les maladies, lésions et désordres qui affligent les êtres humains afin d'en identifier les différentes étiologies, les conditions d'apparition et de développement. Les connaissances ainsi acquises rendent éventuellement possible une intervention médicale adaptée et surtout informée de la nature des forces qui sont à l'œuvre, celles qu'il est possible de mettre à profit et utiliser pour lutter efficacement contre d'autres forces produisant habituellement des effets que nous voulons contrer. C'est l'ensemble de la méthode scientifique qui est enjeu et que devrait adopter la recherche en morale.

Il s'agit de rien de moins en effet que de l'application de la méthode scientifique à la morale humaine. S'il est vrai que la vie morale résulte de contraintes dont l'origine est biologique, et que les formes sociales qu'elle prend sont aussi biologiques puisque la société n'est elle-même qu'une invention, parmi d'autres, de la vie, rien ne s'oppose au recours à la science pour comprendre les régularités présentes dans la morale, et les limitations que celles-ci imposent à la réalisation d'une vie sociale plus conforme à nos désirs. Si, dans notre volonté de nous opposer efficacement à certains comportements jugés immoraux pour tenter de les modifier, nous désirons vraiment faire autre chose que courir au parlement dans une confiance quasi magique dans l'efficacité des mots et des lois répressives, alors il importerait de mieux comprendre la nature précise des forces contre lesquelles nous luttons en morale et qui sont bien autre chose que des mots. La méthode scientifique impliquée est la même que celle qui a fait la preuve de son efficacité depuis maintenant plusieurs siècles. Nous devrions éviter de nous fier à l'efficacité des lois antiracistes pour la même raison qui nous retient d'édicter des lois contre le cancer.

Pourquoi, en effet, évitons-nous de rédiger des lois contre le cancer ? Ce n'est pas seulement parce que, évidemment, les cellules cancéreuses ne savent pas lire, 
mais parce qu'il n'est pas en notre pouvoir d'abolir les constantes de l'évolution. La bonne façon de procéder dans le cas du cancer passe par l'étude expérimentale de son développement, et lorsque l'on découvre que le tabac favorise le cancer du poumon, on élabore des lois contre son usage dans les lieux publics. Le même raisonnement devrait prévaloir dans le cas du racisme. Si l'on voulait comprendre l'étiologie du racisme il serait souhaitable de se poser les questions suivantes et de tenter de leur trouver des réponses dans un cadre expérimental adéquat: nous ne sommes pas tous, toujours et partout racistes, pourquoi certains d'entre nous semblent-ils l'être plus que d'autres et dans quelles circonstances le sont-ils? Quelles sont les causes et quels sont les effets, les conditions nécessaires mais non suffisantes, celles qui sont suffisantes, quelle est la réalité du racisme par opposition à ce qui n'en est que le symptôme, etc. ?

Pour éliminer la variole la médecine fabrique un vaccin qui permet d'injecter la maladie sans en provoquer les symptômes, et c'est ensuite le système immunitaire de l'organisme qui protège véritablement l'organisme contre la pathologie. Y a-t-il de même, demanderons-nous, dans l'ensemble des facteurs impliqués dans le racisme, des forces qu'il serait possible de mettre en œuvre afin d'en réduire ou éliminer les effets indésirables ? Il faut considérer en outre que l'intervention médicale sur la variole chez les humains n'a pas anéanti les causes de la variole, qui est un produit de l'évolution s'attaquant régulièrement aux populations humaines, elle n'a fait que nous protéger contre ses effets. Il faut donc se rappeler que l'élément pathogène demeure présent dans l'environnement, et que lorsque l'on a commencé à vacciner une population il est nécessaire de continuer, indéfiniment, l'injection du vaccin protecteur. (Il est vrai que la vaccination a cessé dans plusieurs pays à la suite de l'apparente éradication de la maladie, mais c'est là un autre problème). Il en ira probablement de même dans le cas du racisme, la réduction ou l'élimination de ses effets indésirables n'en supprimera pas les causes biologiques. Si, en vérité, le racisme a des racines biologiques, cela signifie probablement qu'il appartient à la nature humaine et que le problème qu'il pose ne disparaîtra jamais. Ce n'est pas le genre de problème que l'on peut espérer régler une fois pour toutes pour passer ensuite à autre chose, il promet au contraire de réclamer des études continuelles et une gestion constante.

La troisième raison qui fait de la médecine un modèle intéressant pour une morale expérimentale est le cadre théorique général que la médecine a développé au fil des ans et qui lui permet de distinguer le syndrome, qui est l'ensemble des symptômes d'une affection, des agents de transmissions, des effets secondaires et des causes, bref l'étiologie. Le paludisme, par exemple, est une maladie contagieuse produite par un protozoaire parasite des globules rouges du sang, causant de forts accès de fièvre. Son étiologie fut longtemps incertaine et on l'appelait autrefois malaria (de l'italien mala aria, mauvais air). La médecine mit plusieurs siècles à découvrir que la fièvre présente dans cette pathologie n'est pas la maladie elle-même mais seulement un effet secondaire de l'infection, bien que cette fièvre puisse cependant être mortelle. D'un point de vue médical la fièvre qui 
apparaît dans le paludisme est une réaction normale de l'organisme, il faudrait presque dire "saine » dans le sens que le bon état des mécanismes thermorégulateurs de l'organisme la rend prévisible. Il en va de même, dans d'autres pathologies, du frisson, de la syncope ou de la perte de conscience à la suite d'un choc, de la douleur qui accompagne un traumatisme, etc. C'est ce genre de prévisions qui contribue à faire de la médecine une science.

C'est souvent dans une optique analogue qu'une morale expérimentale devrait aborder l'étude d'une conduite immorale, au sens précédemment donné à l'idée d'immoralité : contrainte primaire non confirmée par une contrainte secondaire, ou contrainte secondaire allocentriste. Le racisme, ou toute autre conduite que nous jugeons indésirable, devrait être envisagé comme la tentative d'un organisme de solutionner un problème dont l'origine est dans l'environnement. De même que la fièvre engendrée par le paludisme n'est qu'une réaction de la thermorégulation, en cela indicatrice de l'existence d'un problème et nonobstant le fait que cette réaction soit inefficace et potentiellement mortelle dans les circonstances, de même le racisme pourrait-il apparaître comme une tentative de solution, que nous considérons par ailleurs indésirable, d'un problème qu'il faudrait d'abord identifier. L'ensemble du problème morale posé par le racisme prend alors une forme classique en recherche expérimentale : 1 ) nous connaissons la tentative de solution, que nous considérons insuffisante ou indésirable ; 2) il faut trouver la véritable origine ou cause du problème ; 3) inventer une solution qui apparaisse acceptable à ce problème, plus humaine et respectueuse des droits des gens ; 4) trouver des moyens propres à la réaliser.

\section{Épidémiologie}

Le racisme n'est pas une maladie au sens de la médecine où une maladie affecte l'individu, c'est plutôt un désordre au sens de l'épidémiologie, qui est une autre partie de la médecine et qui la fait intervenir dans un groupe d'individus. C'est un phénomène de masse qu'il convient d'étudier au niveau des populations au moyen des puissants outils d'investigation de l'épidémiologie.

On ne veut évidemment pas laisser entendre que tout problème moral devrait être traité de la même façon que la médecine traite tel ou tel problème. Il y a bien autre chose en médecine que la thérapeutique du paludisme ou de la fièvre. C'est l'ensemble de la problématique médicale qui pourrait être utile à une science morale expérimentale, problématique formulée de façon telle que certaines contraintes primaires étant connues, certaines contraintes secondaires étant socialement acceptées, le phénomène racisme devienne prévisible. Ce genre d'approche ne sera cependant pas toujours possible, pour des raisons qui seront évoquées à l'instant.

Le quatrième intérêt, et le dernier mentionne ici, que présente le modèle médical pour une science de la morale, est la possibilité d'une identification claire 
des limites de ce modèle. Le terme de modèle est utilisé ici dans son sens le plus élémentaire, qui en fait en pratique l'équivalent d'une métaphore. Les métaphores sont utiles, souvent inévitables, mais leurs inconvénients peuvent apparaître dans l'extension de l'usage qui en est fait. La métaphore est utile mais jusqu'à un certain point au-delà duquel elle devient trompeuse ou fallacieuse. Il n'y a de science que des limites de ses propres concepts, idéalement un concept scientifique contient en lui-même les limites de son usage valide. C'est ce qu'il faut maintenant tenter de préciser dans la mesure du possible.

\section{Les limites du modèle médical}

Les deux sciences de base de la médecine sont l'anatomie et la physiologie. L'anatomie est l'étude des structures des organismes par la dissection, la description des organes et leur disposition. La physiologie est l'étude des fonctions par lesquelles la vie se maintient et qui assurent le maintient de la vie individuelle. La question qui se pose est de savoir dans quelle mesure les deux modes d'approches de ces sciences de base de la médecine sont applicables ou transposables dans une étude de la morale. Dans la mesure, importante, où la morale est influencée par l'existence sociale, il s'agit de savoir si nous possédons, dans notre science sociale actuelle, l'équivalent de ces deux sciences de base de la médecine. Dans le cas de l'anatomie la question ne semble pas poser de difficulté appréciable. On peut en effet supposer que l'ensemble de nos connaissances actuelles en sociobiologie humaine, en éthologie et en sociologie rassemble grosso modo les principaux éléments pertinents d'une anatomie du social humain.

Mais il en va différemment dans le cas de la physiologie pour laquelle nous n'avons aucune science correspondante. La physiologie est la science qui décrit l'intégration et le fonctionnement commun d'un ensemble d'organes, comme par exemple l'ensemble cœur-poumons-reins d'un corps humain et qui sont observables et identiques dans tout corps humain. Aucune science analogue de la vie sociale n'est possible parce qu'il n'existe pas de modèle unique, observable et fixe de la vie en société. L'être humain normal, en santé, existe, mais nous ne connaissons pas de société qui pourrait être dite normale au sens d'une règle. Déjà la vie sociale humaine à elle seule - despotisme, cité-état, aristocratie, démocratie, cueilleurs-chasseurs, etc. - est trop variée pour permettre quelque chose comme une "physiologie sociale » ni par conséquent l'imputation qui voudrait que certaines sociétés particulières relèvent d'une " pathologie sociale ». Quels critères pourrait-on en effet invoquer pour dire que telle ou telle forme de vie collective est une « pathologie sociale »?

De plus, la vie sociale animale, de son côté, présente beaucoup d'autres types de formations sociales inconnues dans la vie sociale humaine. Non seulement il n'est guère de forme de vie sociale humaine qui n'ait été préfigurée d'une façon ou d'une autre dans une forme animale qui en semble le prédécesseur, la vie sociale animale est beaucoup plus riche et variée et connaît des formes qui sont en 
pratique inaccessibles aux êtres humains, comme les sociétés dites despotiques des hyménoptères dans lesquelles tous les membres femelles sauf la reine sont stériles, ou les sociétés-colonies d'invertébrés marins tous nés d'un seul oeuf et donc génétiquement identiques et bien d'autres encore. Il n'existe pas de forme théoriquement canonique, unique, du social, les sociétés animales existantes sont le produit d'une évolution opportuniste qui, dans d'autres environnements, aurait $\mathrm{pu}$ - et peut encore - en inventer d'autres.

Par conséquent l'auteur d'un acte que nous considérons immoral ne peut en aucun cas être considéré comme un malade ou un « malade social » parce que nous n'avons aucune idée de ce que pourrait être la santé sociale ou une société normale et encore moins une société idéale.

Cette limite à l'usage du modèle médical est particulièrement importante pour notre propos, cruciale. L'évolution darwinienne est eugéniste et produit des animaux supérieurs, c'est-à-dire supérieurement reproductifs dans leur environnement; les humains, tout au contraire, dans la mesure où ils sont humanistes et particulièrement par leur médecine, ne sont pas eugénistes et ne sont pas des animaux supérieurs au sens de Darwin : ils guérissent certaines maladies infectieuses et par conséquent empêchent l'élimination des sujets qui y sont les moins résistants, ce qui permet à ceux-ci de se reproduire en même temps que leur susceptibilité particulière à la maladie, ce qui est une façon d'entretenir le problème; ils corrigent les pieds bots, protègent les débiles et les idiots, fournissent des prothèses aux myopes, édentés et estropiés, procurent une aide aux aveugles, aux mutilés et à ceux qui souffrent de divers handicaps qui, dans une société animale, réduiraient les possibilités individuelles de survie et de reproduction. Notre médecine, qui a longtemps été encore plus humaniste que notre morale, les a secourus, et c'est pourquoi les humains qui existent ne sont pas des animaux supérieurs au sens de Darwin.

C'est dans ce contexte que se déroule notre vie morale, qui est assujettie à des contraintes primaires qui sont de deux sortes et que nous considérons de façons très différentes. Il y a en premier lieu les contraintes primaires dont les effets nous plaisent, dont nous sommes fiers et auxquels nous identifions totalement notre réalité, comme individus et comme groupe, nous persuadant que c'est là précisément ce que nous sommes; c'est ce que nos contraintes secondaires appellent nos valeurs. Et en second lieu il y a les autres contraintes primaires, celles dont les effets ne nous plaisent pas, dont certaines nous déplaisent même extrêmement, qui peuvent nous faire horreur et auxquels nous sommes absolument incapables de nous identifier; ce sont les effets de cette seconde sorte de contraintes primaires que nous appelons immoraux. 


\section{L'immoralité est quelque chose de vivant}

Je ne pense pas qu'il existe une seule conduite humaine qui puisse être considérée comme immoralité « en soi », per se, ou par définition. Toutes les pratiques que nous, Occidentaux du $\mathrm{XX}^{\mathrm{e}}$ siècle, considérons comme les plus abominables, ont été pratiquées un jour ou l'autre, par une société ou une autre, et sanctionnées par des autorités politiques ou religieuses appartenant à un autre ethnocentrisme que le nôtre. Notamment la mise à mort massive de populations entières lors du sac d'une ville ; l'esclavage ; l'exécution en masse de prisonniers ou de membres du groupe considérés comme déviants, hérétiques ou autrement indésirables; la torture, subie volontairement lors de rites de passage de l'adolescence mâle à la vie adulte, ou involontairement par des prisonniers ou des déviants ; l'infanticide et la prostitution pratiqués dans le temps ou dans un autre endroit choisi et que nous interprétons de nos jours comme un mode local de contrôle démographique ; la mise à mort du "fils rebelle »; la mise à mort de l'adultère ; l'immolation de la veuve sur le bûcher funéraire de son époux; le suicide commandé par le supérieur hiérarchique.

Il n'y a donc pas d'immoralité en soi, chaque ethnocentrisme définit les formes qu'elle prend pour lui, mais toutes les formes d'immoralité sont des formes de vie. Toutes sont quelque chose de vivant, c'est de la vie qui lutte contre de la vie, c'est une forme de vie qui est jugée mauvaise par une autre forme de vie qui se juge bonne. L'immoralité est un produit nécessaire de la culture, au double sens agricole et social. Le cultivateur qui transforme une parcelle de forêt en champ de maïs ne souhaite produire qu'une chose, du maïs, mais il en produit nécessairement deux : du maïs et des mauvaises herbes. La raison est facile à comprendre, car la culture du maïs n'utilise qu'une partie de l'ensemble des formes de vie présentes dans la forêt vierge, et ce sont les formes inutilisées qui réapparaissent nécessairement dans le champ de maïs en ce qui est alors nommé mauvaises herbes. C'est ainsi que l'on peut imaginer l'apparition de l'immoralité dans la culture au sens social. L’immoralité est une mauvaise herbe sociale mais il n'y a pas de mauvaise herbe en soi, la mauvaise herbe n'est considérée mauvaise que parce qu'elle vit et se propage aux dépens de celle que l'on s'efforce de cultiver et qu'en morale nous appelons une valeur. Il n'y a pas de culture du sol possible sans production de mauvaise herbe. De ce point de vue une société humaine sans immoralité est une impossibilité théorique.

Je résume l'argumentation sur les limites du modèle médical. Aux fins d'une science expérimentale de la morale l'utilité du modèle médical est limitée en ce que ce modèle est lié à la physiologie du corps en santé d'un homme normal. Mais comme il n'y a pas de société normale au sens où il y a un homme normal, il s'ensuit que nous n'avons pas de physiologie sociale d'une société humaine en santé. 
Ce résultat est de grande conséquence en montrant en quoi une science expérimentale de la morale diffère du fonctionnalisme de la sociologie américaine. Le premier principe du fonctionnalisme est en effet que, dans une société, " tout fonctionne », chaque élément quel qu'il soit est réputé jouer un rôle et exercer une fonction que le fonctionnalisme se charge d'identifier. On demandera alors à savoir à quoi sert l'immoralité. En d'autres mots : si tout fonctionne, pourquoi y a-t-il des dysfonctionnalités apparentes dont certaines sont si désastreuses ? Le défaut du fonctionnalisme de fournir à cette question une réponse à la fois observable et universelle suggère que la sociologie qui se désigne sous le nom de fonctionnalisme traduit en réalité la forme privilégiée adoptée par l'ethnocentrisme américain.

Ce que nous conservons du modèle médical peut être résumé en quatre points :

1. C'est une approche interventionniste, humaniste et anti-eugéniste de la vie morale. Elle est expérimentale et tente d'identifier les contraintes secondaires les plus humanistes.

3. En médecine la maladie est considérée comme une forme de vie qui lutte contre une autre forme de vie ; la variole, par exemple, se présente sous la forme d'un virus dont la prolifération peut tuer le corps humain qui en est l'hôte ; la médecine lutte efficacement contre lui en faisant appel au système immunitaire. Une morale expérimentale considérera de même que l'immoralité est une forme de vie, qu'elle tentera de même d'identifier et dont elle tentera ensuite de réduire les effets en faisant appel à d'autres formes de vie.

3. La médecine est exclusivement au service du corps. L'art du médecin atteint ses limites dès que le corps humain semble être en danger ; c'est du moins la théorie, quoique le serment d'Hippocrate promettait de ne jamais provoquer l'avortement. Ces limites de la médecine apparaissent clairement dans les mesures d'internement que le psychiatre se croit obligé d'imposer pour, selon l'adage courant dans les salles d'urgence des hôpitaux, "protéger contre luimême l'individu qui est un danger pour lui-même » : dès que le corps est en danger la médecine a grand-peine à s'empêcher de devenir répressive. La morale doit au contraire se mettre au service de l'être humain tout entier et non pas seulement au service du corps, au service de la liberté la plus grande telle qu'elle a été définie plus haut et qui est l'improbable.

4. La morale expérimentale identifie le rôle, et surtout les limites, des contraintes secondaires que l'être humain est le seul à se donner. Elle met en pratique l'idée avancée plus haut voulant qu'on ne saurait lutter contre un ethnocentrisme qu'au moyen d'un autre ethnocentrisme.

Revenons maintenant sur le premier et le plus important de ces quatre éléments : l'interventionnisme de la médecine. Presque plus personne, de nos jours, 
journaliste, scientifique ou philosophe ne prétend à l'objectivité ; chacun reconnaît qu'il peut tout au plus s'efforcer d'y parvenir. Or, de tous les domaines imaginables où cette difficulté peut se faire sentir la morale est certainement le plus évident. Chaque fois qu'une description contient un aspect éthique on peut s'attendre à vérifier l'adage selon lequel décrire c'est presque toujours prescrire. Cette constante qui relève en dernière analyse de l'ethnocentrisme rend impérative l'adoption d'une approche ouvertement interventionniste dans une science de la morale : puisque la tendance à prescrire est si forte il est non seulement prudent mais impératif de faire face au problème ouvertement et de façon délibérée. Par elle-même cette politique ne rendra pas la description plus transparente ni plus fidèle aux faits mais seulement plus ouverte à la critique et plus aisément falsifiable. Ce qui doit se traduire, en toute recherche faite au nom d'une science de la morale, par une priorité accordée à l'établissement d'un diagnostic.

\section{L'importance du diagnostic}

Par le terme diagnostic j'entends l'énoncé de la décision établissant qu'une situation de fait est moralement indésirable et donc qu'elle pose un problème moral ; un tel énoncé est la première étape de la recherche d'un moyen d'y remédier.

Il est presque impossible d'exagérer l'importance d'une politique d'approche ouvertement interventionniste centrée sur le diagnostic. Une science de la morale devrait adopter une approche tout aussi ouvertement interventionniste que celle que l'on trouve en médecine : tout comme la pratique de la médecine compte sur sa connaissance détaillée de l'évolution des organismes vivants pour appliquer une politique délibérément humaniste et donc anti-évolutionniste et anti-eugéniste ; de même une science morale devrait adopter le même type d'approche lorsqu'elle tente de comprendre et de traiter l'agressivité, le racisme ou toute autre séquelle de l'évolution qui apparaîtrait indésirable. Le diagnostic est de la plus grande importance et l'on devrait consacrer un soin particulier à éviter d'accommoder après coup le diagnostic à la solution culturellement reçue ou habituelle ; prendre l'effet pour la cause est l'une des fautes les plus fréquemment rencontrées en sciences sociales. En théorie l'application de cette règle relative au diagnostic peut sembler ne pas poser de difficulté insurmontable mais nos habitudes culturelles sont si éloignées de cette façon de voir et susceptibles d'opposer une hostilité durable à une telle approche en morale que le développement d'une politique et d'un art du diagnostic moral promet d'être long, ardu et parsemé d'embûches.

Le problème c'est que la principale raison qui nous fait accepter l'idée d'une intervention médicale même agressive c'est qu'elle est faite au nom de la science, même si nous savons par ailleurs qu'en tant qu'elle est aussi un art la médecine a constamment recours à la morale, alors que nous n'avons aucune expérience d'une science de la morale qui autoriserait des interventions analogues parce qu'il n'existe rien à l'heure actuelle en morale correspondant à l'art du diagnostic tel que 
pratiqué en médecine et qui s'obligerait à énoncer ses valeurs, ses objectifs et ses limites.

Pour illustrer le genre de problèmes complexes que l'on peut s'attendre à trouver on peut revoir, dans une perspective plus large que la sienne, la thèse devenue classique de Lionel Tiger dans Men in Groups dans laquelle l'auteur montre que presque partout dans le monde la guerre étant une entreprise réservée aux mâles, c'est l'attachement profond entre les mâles qui assure à la fois et l'une par l'autre la paix à l'intérieur de la société et la guerre au dehors. Selon cette façon de voir le lien très fort établi entre les mâles aurait pour effet d'inhiber l'agressivité potentielle entre eux en la redirigeant contre l'extérieur du groupe, alors qu'habituellement les femmes, de leur côté, ne développent pas d'attachements aussi profonds entre elles ni ne font la guerre. L'auteur résume son argumentation dans les termes suivants :

La logique de mon argument est donc la suivante : les mâles contractent de profonds attachements entre eux; ces forts liens entre mâles favorisent l'agressivité ; donc l'agressivité est un trait prévisible de tout groupe composé de mâles humains. Vouloir réduire le nombre d'occasions qu'ils ont de manifester cette agressivité serait dénaturer [tamper with] un très ancien et fondamental élément de la conduite humaine. Compte tenu de l'importance de la chasse et des conduites qui s'y sont rattachées dans le passé des mâles humains, on peut avancer l'idée que des mâles sans forts liens entre eux et non agressifs sont réellement comme des femmes qui seraient sans enfants ${ }^{1}$.

D'une façon générale cette étude est typique des travaux du genre en anthropologie et le lecteur n'a pas de raison de douter de la véracité des descriptions proposées. Mais en même temps elle représente aussi un cas type de la prétention traditionnelle des sciences sociales à l'« objectivité » et leur refus de traiter ouvertement des valeurs qu'elles favorisent aboutir en un clair cas de description se métamorphosant en prescription. Si l'auteur s'était le moindrement préoccupé d'établir un diagnostic sur la nature précise du problème auquel il était confronté, quelle que fût la nature de ce problème, il aurait été obligé d'énoncer ses propres valeurs et d'établir par la suite les objectifs qui lui seraient apparus désirables ou susceptibles de remédier à ce problème. Il aurait alors probablement mieux su résister à la tentation de présenter les faits comme s'ils étaient l'équivalent d'un destin élevant l'agressivité mâle à la dignité de quelque nécessité historique quasiment ontologique, et de sembler présenter l'absence, chez les femmes, des forts liens établis entre mâles comme l'équivalent de leur destin de machines à enfanter. Chez les animaux la physiologie épouse la forme d'un destin, chez les humains c'est différent. L'auteur aurait alors réalisé qu'en Occident toute activité humaine non seulement peut mais semble devoir fréquemment dénaturer, comme il dit qu'il ne faut pas faire, des tendances établies de longue date dans l'évolution des vivants : l'humanisme est anti-eugénique.

Lionel Tiger, Men in Groups, p. 190. 
Mais en fait toute cette argumentation est faussée à la base et follement moralisatrice car personne au monde n'est en mesure de " dénaturer » l'évolution décrite par la biologie. Cette vérité la plus élémentaire doit donc hélas, encore et toujours, être rappelée. Il est aussi impossible de s'attaquer à l'évolution qu'à un pilote d'avion de violer la loi de la gravitation ; on peut tout au plus s'en servir, ce qui est tout le contraire d'une attaque. Si l'auteur s'était rendu compte de l'importance des valeurs et de l'ethnocentrisme moralisateur affectant ce qu'il appelle la logique de son argument, il aurait pour le moins tenté de retourner son argument contre lui-même et se serait alors procuré l'occasion de s'interroger sur la possibilité que les mâles apprennent un jour à s'enticher des plaisirs d'une agressivité réduite, tout comme les femmes pourraient éventuellement apprendre à être des nullipares heureuses.

Évidemment l'auteur n'énonce pas en toutes lettres son intention d'établir une équivalence entre l'histoire des êtres humains et leur destin mais sa description et son argumentation n'empêchent pas le lecteur de le penser et dans ce genre de texte c'est tout comme. Une bonne partie de la confusion et des déboires engendrés en science sociale par la diffusion populaire du "darwinisme social » résultaient d'une semblable absence de sens critique et d'une complaisance dans l'idéologie dominante de l'époque.

La morale de cette histoire c'est qu'en matière humaine toute description tend à se transformer en prescription et que dans une science morale le développement d'un art du diagnostic serait essentiel.

\section{Langage et immoralité}

Ce qui distingue, on le sait, le primate humain de tous les autres vivants est qu'il dispose d'un accès au langage. Cet accès à l'univers symbolique lui permet de se donner une série ethnocentriste de contraintes secondaires qui réalisent les contraintes primaires, contraintes secondaires dont il fait ses valeurs. Le primate sans langage, au contraire, est sans recours par rapport à l'ensemble de ses contraintes primaires, il leur est soumis et en subit simplement les conséquences.

On sait, par exemple, que chez les primates non humains l'agressivité prend un grand nombre de formes, qui vont des plus bénignes jusqu'à la mise à mort de membres du groupe. Ce dernier cas, et notamment l'infanticide, est rare mais il a néanmoins été observé chez le chimpanzé. Les causes immédiates de cette agressivité peuvent être fort diverses. Mais en biologie sociale, d'un point de vue évolutionniste, particulièrement dans le cas de mises à mort de membres du groupe, l'agressivité sera considérée comme l'une des formes de la compétition décrite par Darwin et interprétée comme une variable dépendante de la densité de la population. Tant que les ressources, principalement la nourriture et l'espace, sont abondantes relativement à une population donnée, l'agression n'apparaît pas et la population peut continuer de croître. Lorsque les ressources deviennent rares, 
l'agression peut apparaître et son premier effet est alors d'abaisser le taux de la croissance démographique ; si elle continue de croître elle finit par rendre cette croissance nulle et à partir de ce point l'agressivité ne peut plus croître parce que son effet s'inverse et augmente en pratique la somme des ressources disponibles à chacun des organismes individuels d'une population qu'elle a déjà commencé à réduire. Le biologiste interprétera de cette façon, ce sera l'une de ses premières hypothèses, les conduites agressives observées chez les primates non humains. On dira alors que l'organisme du primate sans langage est doté de contraintes primaires d'agressivité dont l'un des effets est de commencer à résoudre, dès qu'il commence à apparaître, le problème de la disponibilité des ressources, qui est en pratique le problème de la quantité de ressources accessibles à chaque candidat à la reproduction. Le primate sans langage ne dispose pas de moyens pour s'opposer à l'efficacité de ces contraintes primaires. Il en subit simplement les conséquences et la vie continue.

Le primate parlant, au contraire, dispose de tels moyens par les contraintes secondaires qu'il se donne. Le nombre de stratégies possibles capables de produire le même résultat, c'est-à-dire un contrôle effectif de la croissance de la population par rapport aux ressources, est très grand et varié. Par exemple, il peut sacraliser les sacrifices humains, notamment l'infanticide, au nom d'un « Dieu le veut ! » et le pratiquer sur une haute échelle comme l'ont fait les Phéniciens, peuple sémite qui vivait il y a environ 4000 ans dans la région de Tyr et Sidon du Liban actuel. Cette stratégie est efficace, jusqu'à un certain point, mais ce n'est là qu'une des stratégies humaines possibles parmi d'autres qui peuvent être tout aussi efficaces. La seule chose certaine est la réalité, d'une façon ou d'une autre, de cette contrainte primaire biologique constituant l'essence de ce que Malthus et Darwin nous ont enseigné. Vers la même époque un autre peuple sémite voisin, les Hébreux, peuple pastoral, nomade et guerrier, choisissaient au contraire l'expansion du groupe, avec des règles de mariage prescrivant une endogamie très prononcée et l'interdiction de tout sacrifice humain. Le primate parlant peut aussi favoriser l'homosexualité et la pédérastie comme dans la Grèce classique, dont on trouve des traces encore de nos jours sur l'ensemble du pourtour du bassin méditerranéen. Ou réduire le nombre des géniteurs mâles, avec ou sans infanticide femelle, comme chez les Yanomami de l'Orénoque où l'on rapporte que trente pour cent de la mortalité mâle est attribuable à la guerre. Ou la polyandrie (une femme a plusieurs époux) comme on voit chez certains peuples du nord de l'Inde exploitant une terre ingrate de surface limitée aux pieds de l'Himalaya. Ou, depuis quelques années en Occident, l'usage de contraceptifs efficaces. Ou n'importe quel assemblage de telles mesures produisant le même résultat. Chacune de ces stratégies met en œuvre un ensemble particulier de contraintes secondaires qui deviennent des valeurs, et chacune par conséquent donne un sens particulier et local à l'immoralité.

En termes plus explicites, chez le primate sans langage les contraintes primaires (CP) s'exercent de façon directe ; chez le primate parlant leurs effets passent par des contraintes secondaires (CS) appelées valeurs. Les conduites ainsi 
valorisées sont particulières à chaque ethnocentrisme: là où les Phéniciens pratiquent l'infanticide, les Hébreux ont recours à d'autres moyens pour ajuster leur population aux ressources disponibles. Chacune de ces sociétés aura une façon qui lui est particulière de définir ses valeurs, et par voie de conséquence de définir son immoralité (I). La valeur n'est donc que la face ethnocentriquement visible de la morale, l'immoralité en est le revers, la face moins visible et réprouvée, mais c'est leur ensemble qui constitue la morale humaine totale :

$$
(\mathrm{CP})=(\mathrm{CS})+(\mathrm{I})
$$

ce qu'on pourrait exprimer, en termes plus classiques :

$$
\text { (Morale) }=(\text { Éthique ou Valeurs) }+ \text { (Immoralité) }
$$

Donc la morale humaine se compose de valeurs et d'immoralité. Il est trompeur de parler des valeurs d'une société sans mentionner également ses formes d'immoralité. L'immoralité est le « coût », pour ainsi dire, entraîné par les valeurs acceptées. Par exemple, la Grèce antique a connu une forme limitée de démocratie, mais c'était au prix de l'infanticide (sur une base relativement réduite), l'esclavage et une importante infériorisation des femmes. Il faut donc reconnaître que l'immoralité appartient à la morale. L'immoralité résulte de la contrainte primaire moins la contrainte secondaire

$$
(\mathrm{I})=(\mathrm{CP})-(\mathrm{CS})
$$

ce qui entraîne aussi que la morale effective d'un groupe humain déborde nécessairement les valeurs qu'il affiche. La philosophie morale est toujours en retard sur la vie et ceci est extrêmement important à long terme dans l'étude de la morale des différents peuples qui composent l'humanité

$$
(\mathrm{CP})>(\mathrm{CS})
$$

Toute culture morale humaine produit elle-même l'immoralité propre à son groupe et la reproduit nécessairement comme toute agriculture produit nécessairement de la mauvaise herbe qu'elle doit par la suite se donner les moyens de combattre et extirper par des moyens chimiques, biologiques ou mécaniques dans le cas de l'agriculture, par l'ethnocentrisme, l'école, l'église et la prison dans le cas de la culture morale, en plus de l'art en général et des moyens de communications de masse en particulier. L'immoralité est un produit socialement indésirable, un déchet social si l'on veut mais c'est tout de même le sous-produit d'un ethnocentrisme donné et la production continuelle de ce déchet requiert l'institutionnalisation d'un système adéquat d'élimination. Par exemple, il n'est pas possible d'établir la réelle valeur de la démocratie en Amérique du Nord sans prendre en compte ses formes propres d'immoralité, et cela aux yeux des NordAméricains eux-mêmes. Dans toute société humaine une partie relativement 
importante de la vie sociale est ainsi consacrée à la gestion et à l'élimination des diverses formes d'immoralité accompagnant ses valeurs particulières comme autant de « mauvaises herbes ».

Dans cette façon de voir, l'immoralité joue un rôle important lorsqu'on l'examine à la lumière de l'évolutionnisme. S'il est vrai que les humains sont bien le produit d'une évolution, alors toutes leurs actions, morales et immorales, doivent obéir ou au moins être rendues possibles par des tendances et des modèles hérités de l'évolution. Le développement d'un organisme ne se fait pas au hasard mais selon certains processus biologiques qui relèvent de l'épigénèse et qui lui procurent ses traits caractéristiques. Et selon Lumsden et Wilson ${ }^{1}$ il existe des règles épigénétiques du développement mental. Lorsque ces faits sont interprétés dans le cadre d'un ethnocentrisme et d'une tradition morale particulière, il nous est possible de mieux comprendre, comme il a été dit plus haut, comment chaque culture en vient, sous l'effet des contraintes et des avantages propres à son histoire, à intégrer certaines conduites et actions rendues possibles par l'évolution comme morales et désirables et à interdire d'autres conduites et actions, rendues possibles elles aussi par l'évolution parce qu'elles sont non intégrées et considérées immorales.

C'est pourquoi il faut éviter de considérer l'auteur d'une action immorale comme un « malade social » : la contrainte secondaire produit des déchets, elle les produit nécessairement et elle méconnaît tout aussi nécessairement en être l'auteur parce qu'elle se présente à elle-même comme valeur, rendant ainsi impossible l'établissement d'une filiation entre la vertu morale et la contrainte secondaire d'origine biologique qu'elle est. La contrainte secondaire est un voile d'ignorance, c'est là l'essentiel de l'ethnocentrisme. L'usage fait ici de cette expression, voile d'ignorance, diffère évidemment de celui que l'on trouve dans l'ouvrage de John Rawls ${ }^{2}$ où cette expression désigne une simple convention dans l'exposé de la théorie contractuelle de la justice. Ici, au contraire, le voile d'ignorance est partie intégrante et nécessaire de la valeur.

C'est aussi pourquoi le modèle médical est d'un usage limité pour une science morale expérimentale car la médecine n'a pas produit de tels déchets (ce qu'elle aurait cependant fait si elle avait poursuivi sur une certaine période l'eugénisme des expériences nazies pratiquées sur des sujets humains).

Ceci n'est pas du réductivisme. Je ne dis pas que le primate parlant n'est qu'un primate comme les autres. J'entends seulement qu'il est injustifié et déraisonnable de croire que le développement du langage a fait de nous des non-primates ou des organismes non soumis aux contraintes fondamentales de la vie sociale, ou que la morale n'a pas de fondements biologiques. Si l'évolution nous avait dotés d'un

Charles J. Lumsden, et Edward O. Wilson, Promethean Fire, p. 70.

John Rawls, A Theory of Justice, p. 136 sv. 
organe capable de capter les ondes hertziennes, supposition qui n'est pas abusive puisque la lumière visible que nous captons n'est pas très éloignée des ondes radio sur le spectre électromagnétique, ou encore de l'écholocalisation (en MF !) des chauves-souris, notre monde serait assurément différent mais nous ne dirions pas que ce développement fait de nous des non-primates, une telle affirmation serait déraisonnable. Nous dirions que cela fait de nous des primates capables de capter les ondes hertziennes et qui continuent par ailleurs, sauf indications contraires, à être soumis aux tendances propres aux vivants sociaux qui ont été nos ancêtres, dotés, notamment, d'une nette tendance à la polygamie. Et si, au lieu de faire de nous des cousins du chimpanzé dont la société est de type multimâles, en anglais on dit promiscuous, l'évolution avait produit un primate parlant à partir de la souche génétique du gibbon qui est un primate monogame, les règles de mariage de ce primate parlant montreraient probablement une plus grande tendance à la monogamie que ce n'est le cas pour les humains actuels.

Il faut raisonner de même dans le cas du langage. L'évolution qui a doté le primate humain d'un accès au langage ne l'a pas transformé de ce fait en un nonprimate. Fondamentalement, il y a tout lieu de penser que les contraintes primaires demeurent, et le fait que l'être humain a maintenant de nouvelles contraintes ne signifie pas que les anciennes disparaissent, ou en tout cas nous n'avons pas de raison de penser qu'elles ont été abolies du seul fait que ce primate particulier est maintenant doté de langage. En lui donnant le langage l'évolution a doté ce primate particulier d'une extraordinaire panoplie de moyens pour satisfaire aux contraintes primaires. Ce qui a permis une grande accélération de l'évolution elle-même, le primate parlant pouvant produire en quelques générations un résultat que la sélection naturelle décrite par Darwin pourrait devoir mettre beaucoup plus longtemps à atteindre, si jamais elle y parvenait. Le primate parlant n'est donc pas hors évolution, rien ne nous permet de penser une telle chose, il est au contraire la plus belle réussite à date de l'évolution, les contraintes secondaires qu'il se donne lui permettant de se multiplier sur toute la surface de la terre.

Et je ne dis pas que l'agressivité humaine est à interpréter par les seuls instruments conceptuels dont nous disposons pour l'analyse et la compréhension de l'agressivité chez les chimpanzés. Ce serait particulièrement stupide - impossible, en fait, et c'est un peu pourquoi j'ai choisi l'exemple de l'infanticide chez les primates non humains - car s'il y a un domaine du comportement humain pour lequel la biologie sociale animale se révèle trop courte à elle seule, c'est bien celui qui concerne l'agressivité humaine. Les humains ont poussé leur aptitude à manifester et à tolérer l'agressivité à des sommets et à des raffinements dont les autres primates sont manifestement incapables. Par exemple, les humains sont capables d'une cruauté, d'une banalisation de la violence et d'un plaisir à faire souffrir qui semblent confusément associés au plaisir sexuel comme on voit de nos jours dans la pornographie de masse, et comme on pouvait déjà voir il y a deux cents ans dans le rationalisme forcené des écrits du Marquis de Sade. Cette agressivité humaine constitue un ensemble pour lequel nous ne disposons d'aucun 
moyen d'analyse adéquat à l'heure actuelle. Mais c'est précisément parce que notre incompréhension est si grande qu'il importe de revenir aux connaissances de base dont nous disposons et nous en servir dans une approche expérimentale : le primate parlant est un primate, les contraintes primaires sont la base de la morale et sont toujours suivies d'effet, quelles que soient les contraintes secondaires qu'il se donne et qui ne sont que les valeurs conscientes de sa morale dite, ethnocentriste. Une approche expérimentale de ce problème poserait au point de départ, par principe, que toute manifestation d'agressivité humaine est une tentative de résoudre un problème ; elle identifierait dans chaque cas particulier le problème réel et tenterait d'y apporter une solution plus désirable, plus souhaitable, plus humaniste. Elle tenterait de formuler des contraintes secondaires (CS) mieux adaptées aux contraintes primaires $(\mathrm{CP})$ et donc plus aptes à réduire l'incidence et l'ampleur de l'immoralité (I).

Et l'approche expérimentale mettrait sur le même pied et donnerait le même statut à toutes les «explications » actuelles de la pauvreté de notre maîtrise de l'agressivité humaine: Satan, une insuffisance de prières à l'église ou une insuffisance de lois répressives votées au parlement, l'« instinct » d'agressivité à la Konrad Lorenz ou la prétention positiviste de débarrasser l'humanité une fois pour toutes de la morale au moyen d'une science sociale value-free. Pour le positiviste actuel, la morale c'est toujours la morale des autres, son ethnocentrisme particulier le porte à croire qu'il est lui-même hors morale. Toutes ces interprétations ne sont qu'autant d'extrapolations illimitées de morales historiques dont l'effet est toujours de rendre impossible une recherche expérimentale en morale.

Qui ne souhaiterait, dans ces conditions, se mettre à l'écoute d'une recherche expérimentale qui, sur l'ensemble de la question de l'agressivité humaine, pourrait nous apprendre des choses que nous ne savons pas, ou simplement des idées contre-intuitives ? La méthode expérimentale est la meilleure méthode connue pour nous mettre en état de comprendre ce que nous ne comprenons pas encore. Le plus grand obstacle à une meilleure compréhension de l'agressivité humaine n'est pas que nous avons trop de science, mais pas assez. Natura non nisi parendo vincitur.

Cette analyse confirme, quoiqu'à sa façon, notre idée préconçue selon laquelle la morale humaine est liée à la conscience de faire un " choix ». Chaque société humaine met en effet l'individu en demeure de " choisir », si l'on peut s'exprimer ainsi, les contraintes secondaires imposées par le groupe ethnocentriste et d'éviter l'immoralité que le groupe attache à la pratique d'autres contraintes secondaires. Ce dont la conscience morale n'avertit pas l'individu, cependant, c'est que ce « choix » est en pratique une mise en demeure permanente de reconfirmer son appartenance au groupe par la pratique des valeurs ethnocentristes de ce groupe. La conscience morale n'avertit pas l'individu du fait que le véritable " choix » de ces contraintes secondaires particulières, plutôt que d'autres qui pourraient être tout aussi efficaces, a eu lieu longtemps avant que cet individu n'apparaisse lui-même sur la 
scène de la vie, que ce « choix » est le produit d'une longue évolution opportuniste de son groupe dans sa façon propre, historique, géographique, religieuse et technique de satisfaire aux contraintes primaires communes à tous les vivants sociaux. Ce système semble en général efficace puisque, dans l'immense majorité des conduites humaines, le comportement individuel conforte la valeur du groupe et parvient à éviter l'immoralité telle que le groupe l'a définie : la plupart d'entre nous, la plupart du temps, pensons et agissons comme notre voisin pense et agit. Mais cette efficacité relative se paie par l'ethnocentrisme d'un système de valeurs qui traîne avec lui son immoralité spécifique comme sa face nocturne et maudite. Seule la forêt vierge ne produit pas de mauvaise herbe et seule l'évolution d'avant le langage échappe radicalement à l'immoralité.

\section{D'un centrisme à l'autre}

Il y a là, me semble-t-il, une leçon de choses. Pour être humain il faut communiquer et pour communiquer il faut être d'un lieu, d'un groupe sis dans un espace et un temps qui fournit le répertoire de signes, la syntaxe, la grammaire et les fautes de grammaire, le système de valeurs et l'immoralité. On ne peut pas inclure dans un groupe sans exclure ce qui n'en est pas, on ne peut pas être social et n'appartenir à aucun groupe ou en n'étant de nulle part, ce n'est pas possible. Pour une morale expérimentale, la leçon de choses me semble être que puisqu'il n'est pas possible de sortir d'un ethnocentrisme particulier si ce n'est à partir d'un autre, elle ne devrait pas tenter de sortir du sien mais plutôt choisir d'y entrer délibérément afin d'en mieux préciser les termes, pour identifier les " évidences » propres a son temps et que la suite du inonde révoquera probablement un jour. Tout comme l'historien moderne sait que le piège qui le guette n'est pas d'avoir des préjugés mais au contraire de se persuader de n'en pas avoir et de refuser d'en faire état. Bref, sachant qu'elle est temporelle, la morale expérimentale doit prévoir qu'elle sera temporaire. C'est dans cette voie seulement qu'elle pourrait différer des autres ethnocentrismes qui l'ont précédée, et, en tant que science appliquée, autoriser l'espoir d'une efficacité renouvelée.

Pourtant il semble que le plus vieux rêve du discours cohérent occidental soit d'être de nulle part, alors qu'en fait notre univers moral a un centre qui est notre groupe dans lequel tout observateur est aussi un participant. Je ne saurais parler, ce n'est pas mon affaire, des travaux de la physique moderne et de l'« Interprétation de Copenhague » selon lesquels il n'y a pas d'observateur qui ne soit en même temps un participant. Contraria sunt complementa, disait la formule de Niels Bohr. Mais au simple niveau non scientifique et intuitif qui est le nôtre ici, on peut voir que s'il est un domaine familier à tous les êtres humains et dans lequel tout observateur est nécessairement un participant, c'est bien en morale, et la morale humaine est bien autre chose encore que ce que font voir nos valeurs ou nos règles de conduite morale, préceptes et maximes, elle embrasse la totalité de notre existence. Combien ignorant de notre monde moral serait celui qui n'en connaîtrait que les valeurs affichées ! La circularité de la valeur-qui-a-une-valeur dont il a été 
question précédemment n'est pas nécessairement réfractaire à toute analyse, mais elle offre tout de même une forte résistance, depuis cent cinquante ans, à l'approche qui prétend venir de nulle part.

\section{Les quasi-objets}

Cette résistance vient de ce qu'en morale les objets ne sont jamais que des quasiobjets, puisqu'ils sont fabriques par une pensée historiquement datée et inscrite dans une réalité sociale qu'elle ne dit pas. L'opposition Nature-Culture, par exemple, est au moins aussi ancienne que l'anthropologie, qui ne peut s'empêcher de la désavouer pour ce qu'elle a d'imprécis et qui ne peut cependant cesser de s'en inspirer pour décrire les sociétés humaines. Pour chacun de ces deux termes, nature et culture, les spécialistes ont montré qu'il existe plusieurs centaines de sens différents et cette polysémie qui devrait les rendre inutilisables à des fins scientifiques les rend en fait indispensables au contenu moral que le discours à prétention scientifique véhicule malgré lui. Et, de son côté, le sujet moral n'est lui aussi qu'un quasi-sujet, puisqu'il est soumis à des contraintes primaires qui n'autorisent qu'une autonomie limitée à sa liberté biologique.

Nous savons qu'il n'y a pas de telle chose que la Nature au sens strict, c'est-àdire un état du monde où l'être humain ne serait pas, ni davantage une Culture, c'est-à-dire une liberté non liée à un état du monde. Nous savons que ce que nous appelons le sens est, soit une métaphore encore active et qui rend alors possible notre intervention dans ce monde, soit au contraire une métaphore fatiguée, exsangue, stéréotypée et sédimentée au niveau de ce que nous appelons la « réalité ». Ce que nous appelons la Réalité n'est pas un arbitre impartial dans la diversité des interprétations, c'est l'impératif conventionnel du groupe, c'est-à-dire l'ultime argument où se concentre l'arbitraire d'un ethnocentrisme. Pour notre groupe, la Réalité est le riche humus des anciennes métaphores accumulées sous nos pieds dans la bienveillance de l'oubli. J'aurai beau me battre les flancs tant que je voudrai je ne parviendrai jamais à me convaincre intimement que l'attraction des corps à laquelle s'opposait Leibniz est l'une ou l'autre des choses qu'elle fut pourtant successivement : une simple équation mathématique, une Hypothèse, une Théorie, un Scandale et finalement une Loi ; et je ne réussis pas davantage à croire, comme le pensait Newton, que le temps est un organe de Dieu. La gravitation, c'est la Réalité, n'est-ce pas? Il m'est impossible de penser autrement. Ce que nous appelons la réalité est la métaphore refroidie d'une description convenue. La vérité de la métaphore c'est le groupe, dans tous les sens du mot vérité : l'origine de la chose, le constat d'une identité c'est-à-dire l'affirmation que deux choses aperçues n'en forment «en réalité » qu'une seule, le conventionnalisme qui fait de ce constat la finalité ou la destination promise au groupe.

C'est ainsi que le primate parlant peuple son univers de quasi-objets alourdis des contraintes qu'il assigne à chacun et dont il subit en retour les effets et 
transforme en idoles, et d'autre part de quasi-sujets auteurs de ces quasi-objets et dont il affirme, avec quelque présomption, que rien de ce qui les concerne ne lui est étranger ; en fait tout ce qui déborde son ethnocentrisme lui est étranger et peut lui apparaître comme les superstitions particulières d'un primate à peine humain. Son univers c'est aussi l'ensemble des objets techniques qu'il fabrique, le monde familier de ses quasi-objets techniques, culturels, scientifiques, artistiques. Notre temps devrait nommer " industrie de la conscience », j'entends l'industrie de la conscience morale, l'ensemble de ces activités dont le principal objectif est moins la production d'objets que le modelage et la modification des comportements humains impliquant l'éducation et la rééducation permanentes des membres du groupe, une bonne partie de la science et tout le monde psy, le spectacle, l'art, la littérature, la mode, la publicité, la politique, la religion, les services de santé et les services sociaux, la presse et le monde de l'information, l'administration de la justice. Ce sont là autant de moyens variés de réaliser l'ensemble du peopleprocessing qui assoit et accomplit les contraintes secondaires ou valeurs du groupe.

L'ensemble de ces quasi-objets constitue notre réalité et requiert une éducation de la perception qui vienne sans cesse confirmer la description du monde ayant cours dans notre groupe. Comme dit le personnage Don Juan dans Le Voyage à Ixtlan de Carlos Castaneda l'enfant devient membre à part entière du groupe « lorsqu'il est capable de faire toutes les interprétations perceptuelles qui, parce que conformes à cette description, la valident ${ }^{1}$ ».

\section{La question des objectifs d'une science morale}

Nous devrions, en ce point, énoncer les objectifs qu'il convient d'assigner à une science morale. On pourrait penser spontanément que toute entreprise morale quelle qu'elle soit ne peut avoir qu'un seul objectif qui serait d'accroître indéfiniment la liberté individuelle. Pourtant une telle interprétation n'est pas possible parce que cet usage du mot liberté fait retour à un sens dont toute la démonstration précédente a montré les inconvénients. La tentation d'un tel retour demeure forte et permanente, cependant, normale même compte tenu des habitudes consacrées par deux siècles d'histoire et de philosophie occidentales. Pourtant cette tradition est fautive, dirons-nous, et nous éviterons de concevoir la liberté comme une absence ou comme une réduction de la contrainte puisque c'est le contraire qui est vrai : la morale est une contrainte qui vient réduire la liberté humaine. Assigner à la morale le rôle d'accroître indéfiniment la liberté serait retomber dans la conception qui fait de la liberté une vie sans contrainte et qui confère à la morale locale le monopole du bien. Nous devons au contraire nous en tenir aux définitions proposées : la liberté c'est la vie et la morale est une série de contraintes imposées à cette liberté.

Carlos Castaneda, Journey to Ixtlan, p. 2. 
Nous savons néanmoins que la liberté de la personne, je l'appellerai l'autonomie de la personne, peut parfois être accrue, dans certaines circonstances et jusqu'à un certain point. Il est indéniable que la démocratie, par exemple, a historiquement rempli un tel rôle. Mais ce genre d'accroissement n'a lieu que dans certaines conditions qui ne sont pas évidentes et qui doivent être spécifiées si nous voulons éviter de retomber dans la conception traditionnelle faisant de la liberté une vie sans contrainte. Cette difficulté à laquelle nous sommes confrontés est si grande qu'il nous faut réexaminer à nouveau l'idée de liberté en rapport cette fois avec un autre concept qui représente le principal obstacle théorique à une compréhension occidentale de la morale, la composante la plus importante de la morale et aussi la plus négligée: le groupe. La question des objectifs devra attendre et sera traitée au chapitre 5 dans la perspective plus vaste de la morale humaine. 


\section{Chapitre 3}

\section{Le grand oublié : le groupe}

\section{Liberté}

\section{$\underline{\text { Retour à la table des matières }}$}

Je suis conscient que l'idée de liberté mise en œuvre ici est fort différente de l'acception habituelle de ce terme en Occident. Cette difficulté est incontournable et il est nécessaire de s'y arrêter.

Dans la conception occidentale habituelle, la liberté est présentée comme un mode de vie sans aucun obstacle, entrave ou empêchement d'aucune sorte. Cette idée me semble inspirée d'une version particulière du christianisme qu'il faudrait dire " anticorporelle », le genre d'idée que seul un être immatériel ou spirituel pourrait avoir. Car il n'y a pas de vie sans contrainte, quand ce ne serait que celle de la reproduction ou de l'alimentation, ou, pour les vivants sexués, la contrainte de la mort.

Voici une formulation plus succincte : la liberté c'est tout ce que vous avez toujours pensé que c'est et infiniment plus encore. La liberté c'est tout ce qui est vie. Improbable.

La liberté telle qu'entendue ici est l'affaire du corps, ce n'est pas un idéal qui serait à réaliser dans un avenir promis, toujours à venir et jamais advenu, c'est au contraire le mode d'existence immédiate du corps, de ce qu'il est et de ce qu'il fait. La liberté c'est la vie elle-même et c'est tout de suite, c'est l'autonomie de l'organisme dans son environnement. La liberté c'est l'ordre que la vie introduit dans le désordre de la physique. Du plus simple procaryote à Ludwig van Beethoven toute forme de vie est un ordre au sens de la physique classique et donc relativement improbable, la néguentropie étant fonction de la complexité de l'organisme concerné : plus l'organisme est complexe, plus il est autonome et libre. 
Mais cette liberté est encore prémorale. L’accès à la vie morale suppose encore deux conditions indépendantes l'une de l'autre $: 1$ ) la vie sociale, qui vient réduire le plein exercice de cette autonomie du vivant individuel. Exemple : chez certaines abeilles, l'ouvrière individuelle a conservé la capacité physiologique de se reproduire mais le groupe social auquel elle appartient l'empêche matériellement ou physiquement de l'exercer et détruit ses œufs lorsqu'elle réussit tout de même à pondre. Il en va de même chez certains ongulés, où un grand nombre de mâles physiologiquement aptes à se reproduire en sont matériellement empêchés. Ce sont là des exemples de contraintes proprement sociales imposées à l'autonomie du vivant individuel. 2) La deuxième condition à l'apparition de la vie morale est, on le sait, la communication symbolique humaine par laquelle la contrainte secondaire prend le relais de la contrainte primaire et la réalise dans l'infini du langage. La contrainte secondaire «relance», pour ainsi dire, amplifie prodigieusement, le nombre de façons possibles de réaliser la contrainte primaire, elle est un extraordinaire multiplicateur de liberté. La contrainte secondaire est donc bel et bien une forme de cet infini auquel nous, en Occident, pensons quand nous l'appelons une valeur, mais la liberté qu'elle réalise n'est pas toute la liberté. Cette liberté humaine n'est certainement pas une absence de contrainte ; il est vrai qu'elle est plus grande que celle de tout autre mammifère social ou solitaire et plus grande que celle de tout autre vivant, mais il y a tout de même des formes de liberté biologique qui sont en dehors d'elle et elle-même n'est, très précisément, que la liberté de l'espèce homo, (ordre primates, sous-ordre anthropoïdes, infraordre catarrhini, famille hominidés). Cette liberté a un fil à la patte qui lui est imposé par le groupe.

\section{L'importance du groupe}

Aux fins d'identification des objectifs d'une science morale expérimentale et appliquée, le groupe constitue l'obstacle majeur et permanent, l'ennemi juré de toute science parce qu'il est le grand oublié de notre compréhension actuelle de la morale. Il y a plusieurs raisons à cet état de choses. En premier lieu, et cette première raison s'applique à l'ensemble de l'humanité et donc à toute version locale de la morale humaine, le groupe institue sa propre définition de la réalité à partir de son ethnocentrisme qui semble infranchissable.

Mais il y a une autre raison plus déterminante encore de l'importance du groupe, elle est spécifique à l'Occident car c'est précisément sur ce point que la morale occidentale diffère de toute autre. Les autres morales humaines connues ont tendance à faire prédominer le groupe sur l'individu : à lui seul l'individu n'est rien, le groupe est tout. C'est possiblement notre indifférence au facteur groupe qui fait que, bien que la morale et le mysticisme d'inspiration orientale comme le zen et les autres formes du bouddhisme ont pu faire d'impressionnantes percées en Occident depuis un quart de siècle, ils n'ont pas vraiment développé de racines locales profondes ni ne sont devenus un élément majeur de la culture d'Occident : la moralité d'Orient est fondamentalement une morale de groupe. 
Voici un autre exemple. Du point de vue occidental il est certain que la prise d'otages occidentaux, leur cruelle condition de détention et parfois leur mise à mort au cours de la guerre civile qui a ravagé le Liban de 1975 à 1990 sont des actes immoraux et des abominations. D'un point de vue musulman, cependant, il est possible que ces actions soient apparues vertueuses et qu'elles constituaient l'accomplissement d'un devoir moral pour le croyant qui les posait. Le même genre de problème se pose dans le cas de la sentence de mort prononcée en 1988 par les autorités religieuses d'Iran contre le Britannique Salman Rushdie auteur des Satanic Verses.

Plusieurs personnes, y compris des musulmans, voudront sans doute manifester leur désaccord profond avec ces actions et insister pour dire qu'elles sont immorales. On peut très bien le reconnaître tout en continuant de refuser de tenir compte de cet aspect de la question au profit d'une autre question tout à fait différente. La seule chose qui nous intéresse ici est que ces actions ont eu lieu, leur réalité historique est établie et appelle par conséquent une explication articulée : pourquoi ces actions ont-elles eu lieu ? Il y a nécessairement eu une conscience morale ou un sens moral particulier motivant de telles actions et ce sens doit être compris et intégré dans notre savoir commun sur ce qu'est la morale. Nous ne devons pas permettre à notre désapprobation moralisatrice de nous distraire de cette recherche.

Je ne dis pas que les musulmans sont « différents » et que les non-musulmans devraient automatiquement respecter leur différence. Je dis que la morale musulmane diffère sur un point fondamental de la morale occidentale de tradition individualiste et chrétienne du fait qu'elle est à base « groupale ».

Il existe de nombreuses sociétés humaines où la morale est à base groupale et dans lesquelles des idées occidentales comme l'idée de progrès (en général), d'émancipation de la femme et d'épanouissement personnel, ce qu'aux États-Unis on appelle "le droit à la poursuite [individuelle] du bonheur », peuvent être interprétées à des degrés divers comme autant de formes d'immoralité.

Ce dont nous avons besoin c'est d'une problématique de la question morale humaine permettant l'examen et la discussion de questions semblables qui ne dégénère pas rapidement en condamnations réciproques. Ce dont nous avons besoin c'est d'une compréhension adéquate de ce qu'est la morale humaine, de telle sorte que la morale musulmane, ou tout autre morale fortement basée sur le groupe, soit intégrée à un ensemble dans lequel elle tienne sa place, que son apparition devienne prévisible dès que ses conditions de possibilité (qui restent à identifier) deviennent connues, et que les personnes partageant une telle vision de la moralité humaine soient considérées comme des êtres humains à part entière et non pas des «primitifs » ou des sous-humains « en voie de devenir civilisés ». Les musulmans sont des personnes, et tant que nous ne réussissons pas à intégrer de 
plein droit la morale musulmane dans notre compréhension de ce qu'est la morale notre compréhension n'est pas à la hauteur de sa tâche.

\section{Les religions comme groupes}

On doit cependant reconnaître que la plupart des religions, toutes dénominations confondues - et certainement toutes celles que l'on trouve en Occident - présentent un obstacle particulièrement redoutable à l'établissement d'une telle conception en matière de morale. Les religions, pratiquement toute religion connue, favorisent la plupart du temps une sorte de super-ethnocentrisme luttant pour la conquête souvent appelée « le salut » de l'humanité toute entière ; lorsqu'elles ne manifestent pas une telle ambition, comme dans la religion juive du "peuple élu», c'est l'exclusivisme propre à la forme adoptée par leur ethnocentrisme qui leur épargne cet effort et leur fait réserver les bienfaits de leur pratique à leur propre ethnie. Bien qu'aucune religion ne soit universelle bien peu échappent à l'ambition de le devenir, et bien peu ont réussi à éviter à l'occasion la commission d'exactions et parfois les plus grandes atrocités que l'histoire humaine ait enregistrées. Aussi, bien qu'on doive reconnaître que la religion est un authentique facteur d'union entre les personnes qu'elle regroupe en son sein il faut aussi reconnaître que laissée à elle-même cette entente que la religion réalise entre ses membres est fréquemment acquise au prix d'une intolérance à l'égard de quiconque n'appartient pas au groupe. En général, y compris en Occident, la religion peut en venir à considérer que le plus grand chauvinisme en matière de croyance et de pratique culturelle est non seulement légitime mais constitue un véritable devoir moral et l'accomplissement d'un ordre d'origine divine. Comme si chaque Dieu instituait le groupe particulier de ses dévots comme l'espèce humaine elle-même en sa totalité. En tant que phénomène culturel la religion est la mère de l'ethnocentrisme.

En biologie cette sorte d'exclusivité territoriale, appelée allopatrie, fait que chaque espèce différente occupe un territoire différent : la plupart des religions sont allopatriques dans le sens qu'elles recherchent habituellement de façon active à s'assurer l'usage exclusif du territoire qu'elles occupent. Il existe de nombreuses exceptions apparentes à cette règle, comme le shintoïsme au Japon où une proportion importante des fidèles pratiquent en même temps les rites d'autres religions; mais ce partage pacifique des pratiques religieuses demeure tout de même au cœur de l'ethnocentrisme japonais. En vérité, d'un point de vue biologique social peu d'institutions humaines, de tous temps et de tous lieux, semblent à la fois si exclusivement humaines et si étonnamment animales.

\section{Le groupe et la tour de Babel}

L'extraordinaire multiplication des langues est un autre indice possible de l'influence du groupe pour lequel nous n'avons guère d'explication plausible. George Steiner s'est interrogé dans son ouvrage Après Babel sur l'origine de cette 
prodigalité et sur le fait que si peu de linguistes modernes se soient penchés sur cette question. Pourquoi, demande Steiner, l'homo sapiens ne parle-t-il pas une seule langue ? Ou encore un petit nombre de versions apparentées de la même langue ? La question semble d'autant plus pertinente, dit-il, qu'il n'existe qu'un seul cerveau humain, considérant également le très petit nombre de types de squelettes et de groupes sanguins connus en anthropométrie, moins de dix dans chaque cas sur toute la terre, Et pourtant, ajoute Steiner, quatre ou cinq mille langues sont effectivement Parlées à l'heure actuelle, lesquelles ne sont que les vestiges d'un nombre beaucoup plus grand encore de langues qui sont disparues. Certaines des langues parlées à l'heure actuelle ont une aire de distribution très restreinte et sont maintenues en vie par des groupes d'à peine un millier de personnes. L'auteur estime qu'une véritable science du langage devrait être en mesure de rendre compte de la création et de la stabilité de cinq mille à dix mille langues ${ }^{1}$.

S'interrogeant sur les causes possibles d'une telle abondance il rejette avec raison l'hypothèse d'une évolution de type darwinien qui, par le truchement de quelque mécanisme de sélection naturelle, aurait multiplié les langues à la façon de l'évolution multipliant les formes de la vie sur terre. D'un point de vue biologique on imagine mal en effet l'existence d'une évolution par voie non génétique ou purement culturelle, ce qui est l'essence même du langage.

La question posée par Steiner semble d'autant plus pertinente que cette apparente prodigalité, cette abondance de langues différentes entraîne manifestement des coûts ou des inconvénients considérables en termes économiques, culturels et sociaux. Que l'on songe à la multitude des traductions que cette abondance de langues différentes rend nécessaires et qui sont si souvent inexactes de toute façon; que l'on songe aux guerres que les différents groupes ethnolinguistiques s'infligent les uns aux autres, aux empires militaires qui se font et se défont et qui furent si souvent dans le passé la cause aussi bien que l'effet de tant d'incompréhension, d'absence de confiance mutuelle, d'entêtements et de haines et dont les conséquences prennent trop souvent la forme de quelque « nettoyage ethnique » au service d'une allopatrie ethnocentriste.

On ne peut s'empêcher de supposer que la simple existence de tels inconvénients entraînés par la multiplication persistante et inexpliquée des langues dans l'histoire humaine permet de supposer l'existence de quelque puissant obstacle susceptible de faire dépendre la survie et la reproduction de la culture humaine d'avantages conférés à un bénéficiaire qu'il importerait d'identifier. Se pourrait-il que ce bénéficiaire soit le groupe ? Si cette supposition s'avérait et même si le groupe n'était pas la seule forme de vie à profiter de cet avantage, cela signifierait que nous devrions porter au groupe une attention beaucoup plus grande que celle que nous lui avons accordée jusqu'ici car il s'ensuivrait la nette possibilité que, un peu comme la religion, l'influence que la langue possède sur la vie du

\footnotetext{
George Steiner, Après Babel, p. 60.
} 
groupe en fasse une cruelle maîtresse des humains parce qu'elle n'assurerait alors l'unité à l'intérieur du groupe qu'au prix d'une inimitié permanente à l'égard de tout ce qui lui est extérieur. La langue ne se contenterait donc pas d'être le véhicule de l'ethnocentrisme, elle en serait aussi l'un des plus fermes soutiens. Cette perspective est affligeante. C'est peut-être ce que signifiait, à sa façon, l'idée de Wilhem von Humboldt que personne n'avait avancée avant lui, dit Steiner ${ }^{1}$, à l'effet que la langue peut parfois agresser l'être humain lui-même.

Il serait possible d'interpréter cette situation d'un point de vue différent en ayant recours au concept de «milieu intérieur» de Claude Bernard. Le premier environnement de la vie des animaux supérieurs, explique Bernard, est l'ensemble des liquides dans lesquels les divers organes et viscères se développent et opèrent, relativement protégés des grandes variations climatiques produites dans le second environnement, celui qui se situe à l'extérieur du corps. Dans les organismes complexes, dit Bernard, c'est la stabilité de l'environnement intérieur qui rend possible leur relative autonomie dans l'environnement extérieur. Il explique, dans ses Leçons sur les phénomènes de la vie communs aux animaux et aux végétaux, publiées en 1878, que le rôle du milieu intérieur d'un organisme, animal ou végétal, est de le protéger contre les variations excessives du milieu extérieur « de façon telle que son équilibre interne résulte d'une continuelle et délicate compensation établie comme par la plus sensible des balances ${ }^{2}{ }^{2}$. À notre époque et en partie grâce à lui le concept d'homéostasie joue un rôle central en biologie.

On en vient alors à concevoir que l'organisme complexe est essentiellement un contenant ou une structure rendue possible par le développement d'une sorte de paroi invaginante, une membrane filtrante réduisant l'effet déstabilisateur des turbulences d'un environnement extérieur au moyen de la stabilité d'un environnement intérieur, protégeant l'intégrité de l'organisme jusqu'à une certaine limite, tout en laissant place à des interventions limitées sur ce même milieu extérieur.

De ce point de vue, on considérera que le groupe linguistique créé par le développement de chaque langue particulière joue le rôle d'un environnement intérieur relativement constant, bénéfique au groupe qui utilise cette langue et reposant sur l'exclusion des représentants de l'espèce homo sapiens utilisant d'autres langues qui sont alors considérés comme partie intégrante de l'environnement extérieur, isolant et protégeant relativement de la sorte les membres de chaque groupe linguistique des interventions gênantes des étrangers, posant probablement aussi des entraves à l'échange de leurs gènes, tout en

1 Op. cit. p. 87. Von Humboldt avait dit : «Bien que la langue soit totalement intériorisée, elle possède cependant simultanément une identité externe autonome qui assaille l'homme luimême. »

2 Claude Bernard, Leçons sur les phénomènes de la vie communs aux animaux et aux végétaux, $\mathrm{p}$. 114. 
permettant aux membres de chaque groupe la poursuite des affaires courantes, l'exploitation des ressources disponibles et diverses interventions concertées sur la vie des groupes avoisinants.

Ce point de vue pourrait peut-être nous aider à mieux comprendre l'inexplicable prolifération des langues humaines, mais le raisonnement qui le ferait valoir serait considéré comme très inhabituel, pour dire le moins, selon les normes courantes du raisonnement en biologie. Demandons-nous pourquoi un tel raisonnement serait mal reçu. Ce raisonnement n'a rien de téléologique, il n'avance pas l'idée que les gens « inventent » des langues pour telle ou telle raison, quoique les amants, les poètes et autres révolutionnaires puissent se complaire à de tels exercices sans conséquences, je demande seulement à savoir pourquoi l'invraisemblable nombre de langues humaines passées et présentes semble présenter un tel défi au bon sens et à toute apparente fonctionnalité. Comment peut-on penser comprendre et rendre compte de la persistance et du coût social d'une telle multiplication ? Il semble que ce qui gêne dans la formulation de l'hypothèse avancée concerne l'usage qui est fait du concept d'environnement intérieur de Claude Bernard. On reconnaîtra volontiers que le concept de milieu intérieur a un sens au niveau de l'organisme individuel, mais on ajoutera qu'il est inapplicable à des groupes humains. Par conséquent la véritable objection que l'on oppose alors à la formulation de l'hypothèse est la vieille scie : "Ou bien ce phénomène relève de la biologie ou bien c'est du social. » En d'autres mots : les concepts de la biologie sont considérés pertinents tant qu'il s'agit d'organes et de viscères, mais dès qu'il s'agit d'êtres humains et de leurs groupes il ne doit plus être question de biologie. On pourrait imaginer de nombreuses raisons susceptibles de faire rejeter l'hypothèse avancée au niveau intuitif, mais cette objection particulière ne tient pas.

Cette hypothèse n'est qu'une autre façon d'illustrer l'idée proposée dans le chapitre 2 sur la possibilité que la morale soit l'équivalent fonctionnel d'un système immunitaire du groupe. Selon cette suggestion l'une des fonctions de la morale dans le groupe serait de lier d'autant plus fortement entre eux les membres du groupe qu'elle réoriente leur hostilité sur les personnes extérieures au groupe comme si elles étaient autant de corps étrangers dont la présence présenterait des dangers pour le groupe. L'extrême abondance des langues différentes pourrait, sinon confirmer, tout au moins corroborer cette idée, alors que les autres explications possibles demeurent presque inexistantes.

\section{La morale occidentale}

D'un point de vue rationaliste occidental on serait en droit de s'attendre à ce que, contrairement aux morales non occidentales fondées sur la dominance du groupe et donc ouvertement portées à l'ethnocentrisme ou au chauvinisme, la morale occidentale prétendument fondée sur la liberté du sujet individuel échappe à ce travers de la dominance du groupe et de l'ethnocentrisme. Pourtant il est loin d'être 
évident que la culture occidentale est moins ethnocentriste que les autres : pourquoi les Occidentaux sont-ils racistes ? C'est ce qui nous oblige à reconsidérer l'ensemble de la question de la nature de la morale occidentale, et, en dépit de la vénération que nous disons avoir pour les droits de la personne, reconsidérer l'importance permanente du groupe. Si les Occidentaux étaient incapables de racisme ou d'ethnocentrisme, alors nous pourrions croire que leur morale est vraiment basée sur l'individu comme elle prétend l'être et non pas sur le groupe. Mais ce n'est pas le cas et les Occidentaux, sinon en théorie du moins en pratique, se montrent au moins aussi doués que quiconque en matière de racisme. Il faut donc reconnaître que, bien que l'évolution de la morale occidentale depuis au moins la Révolution américaine, la Révolution française et la philosophie rationaliste kantienne lui ait fait découvrir une dimension jusque-là inconnue de la morale, celle des droits de la personne, nous devons aussi nous rappeler que ce développement n'a pas aboli le rôle du groupe mais en a néanmoins supprimé toute trace dans la théorie. Les droits de la personne ne sont que la nouvelle (dans l'histoire de l'humanité) contrainte secondaire réalisant la contrainte primaire imposée par le groupe et les effets de cette dernière sont encore très actifs et réels sur le sens moral d'Occident. Nous, les Occidentaux, affichons un grand respect pour les droits de la personne, mais cette vénération est notre point de ralliement, c'est là le drapeau de l'hégémonie culturelle occidentale autour duquel nous rallions les nôtres et que nous utilisons pour identifier nos vrais amis, c'est la règle que notre groupe applique et exige que ses membres respectent s'ils veulent éviter la prison ou subir quelque autre traitement tout aussi désagréable.

Suivant cette ligne d'analyse, on reconnaîtra deux grandes sortes ou versions de la morale humaine existant à l'heure actuelle selon le rôle que chacune attribue au groupe. Nous trouvons, d'une part, la morale humaniste à base personnelle typique des sociétés occidentales ou industrielles; selon cette morale l'individu est l'unique origine de la valeur et la vie humaine individuelle est sans prix. Et d'autre part la morale à base de groupe selon laquelle c'est le groupe ou la communauté qui est à l'origine de la valeur : le groupe est tout et hors du groupe l'individu n'est rien. Cette deuxième version, la plus ancienne et celle qui possède de loin le plus grand nombre d'adeptes (les deux tiers, peut-être, de la population humaine actuelle), se trouve dans les cultures et les régions suivantes : le peuple juif dans la mesure où sa morale demeure identique à celle de l'Ancien Testament ; l'Afrique actuelle non chrétienne et non industrialisée, l'Islam qui compte maintenant plus d'un milliard de fidèles dans le monde ; l'Inde classique, la Chine classique, le Japon classique et une grande partie de l'Asie classique, le terme "classique » désignant ici la partie de ces sociétés qui a échappé à l'influence de l'Occident ou qui a su lui résister ; enfin plusieurs sinon toutes les populations autochtones d'Amérique.

On doit supposer que cette morale basée sur le groupe continue d'être présente chez tous les peuples de la terre. Ce n'est que chez quelques-uns, dits démocratiques, que la morale basée sur les droits de la personne s'y est adjointe depuis deux siècles, avec tous les ratés et lacunes que l'on sait : abus de pouvoir, 
concussion, favoritisme, fraudes électorales, népotisme, prévarication, racisme, sexisme, etc. La règle démocratique demeure fragile, toujours précaire, alors que la règle de la morale fondée sur le groupe demeure toujours présente et prête à prendre le relais en cas de défaillance de la première. Il existe en effet de nombreuses situations (état de guerre, insurrections, conflits sociaux graves, catastrophes naturelles, épidémies, dictature) où le pouvoir civil juge bon de supprimer l'exercice des libertés fondamentales telles le droit d'association, d'expression, de réunion, l'inviolabilité du domicile, la sûreté de la personne, etc. occasionnant ainsi un retour à la morale fondée sur le groupe ; on se rappelle, encore fraîche à notre mémoire, l'arrivée au pouvoir des Adolf Hitler, Joseph Staline et autres tyrans. Il faut donc conclure que puisqu'en politique la démocratie ou société de droit peut toujours être abrogée, la morale à base personnelle qui se fonde sur elle demeure tout aussi révocable, alors que la morale fondée sur le groupe ne l'est pas.

On ne veut évidemment pas laisser entendre que toutes les morales fondées sur le groupe qui existent à l'heure actuelle sont semblables ou qu'elles reviennent au même, mais plutôt que chacune d'elles, de son point de vue particulier, est en droit d'affirmer que l'individualisme accusé de la morale occidentale lui fait nécessairement méconnaître la dimension groupale de la morale et que cette lacune est une faute fondamentale. C'est donc de ces sociétés et de leur morale de groupe dont il faudrait tenir particulièrement compte si nous voulions continuer à observer le critère d'universalité énoncé précédemment. La Grèce du temps d'Hippocrate et Aristote appartenait à ce second type. La morale de ces sociétés n'est pas du genre que nous appelons humaniste. Seul l'Occident, depuis deux siècles seulement et d'une façon très inégale affirme que la base fondamentale de la morale se trouve dans le sujet et dans la conscience de la personne individuelle.

\section{La morale d'Aristote}

On peut le voir par un rappel de la morale grecque telle que formulée par Aristote et qui, de toutes les morales groupales, est certainement l'une des plus familières à l'esprit et la culture d'Occident. Dans la morale d'Aristote il n'y a pas de concept de sujet au sens moderne, c'est-à-dire qu'on n'y voit pas l'être humain comme doté d'un intérieur, d'un « libre arbitre » seule origine de la valeur.

En termes aristotéliciens tout comme en termes modernes, mais pour des raisons très différentes, une science de la morale est considérée impossible. Notre théorie moderne de la morale est fondée sur la distinction entre les faits et les valeurs. De nos jours, il est vrai que, selon Hillary Putnam, cette distinction «devient décidément floue puisque les propositions portant sur les faits [...] présupposent elles-mêmes des valeurs ${ }^{1}$ ». Néanmoins, ajoute Putnam, « il n'existe

Hillary Putnam, Reason, Truth and History, p. 128. 
aucune possibilité d'une science de la morale, ainsi qu'Aristote l'a depuis longtemps remarqué (Éthique de Nicomaque, 1,3$)^{1}{ }^{1}$.

Mais dans ce passage bien connu (1094a) au début de l'Éthique de Nicomaque, Aristote écrit :

[La morale étant la recherche du bien] il faut nous efforcer de préciser, même d'une manière sommaire, la nature de ce bien et de dire de quelles sciences ou de quels moyens d'action il relève. Il peut sembler qu'il relève de la science souveraine et au plus au point organisatrice. Apparemment, c'est la science politique ${ }^{2}$.

On peut voir que la raison alléguée par Aristote pour affirmer l'impossibilité d'une science de la morale est très différente de la nôtre : il dit qu'un traité sur l'éthique fait partie d'un traité sur la politique. Ce qui apparaît incompréhensible à des Occidentaux modernes qui pensent exactement le contraire, à savoir que pour eux la politique est l'art et la science de la chose publique et ne peut en aucun cas régir la morale mais doit au contraire être régie par elle. Dire que la morale doive être assujettie au politique apparaît de nos jours une énormité, en fait cela semble la recette même de l'avilissement de la chose publique et de l'inconduite personnelle. Comment Aristote peut-il dire une chose pareille?

De quelque façon qu'on la considère cette difficulté apparaît insurmontable. C'est précisément en ce lieu et sur cette question que l'on trouve la différence la plus grande entre les deux grandes versions de la morale humaine, c'est là qu'est le cœur de la question et notre principal problème sur les relations entre la morale personnelle et la morale de groupe : la morale aristotélicienne, et en pratique toute morale passée ou présente fondée sur le groupe, méconnaît le sujet et le « libre arbitre " tels que nous entendons ces concepts à notre époque et les mettons au centre de notre conception de la morale en Occident. Le concept de «libre arbitre » n'apparaîtra que quatre siècles après Aristote, dans le Manuel d'Épictète qui date de l'an 120 de notre ère et dans lequel il se trouve mentionné cinq fois ${ }^{3}$. Selon Jean-Pierre Vernant et Pierre Vidal-Naquet la langue grecque du temps d'Aristote ne possède même pas de mot pour dire la "volonté » ou le choix délibéré. Cette carence, ou plutôt cette absence, est le fait du groupe qui n'est ni un Sujet ni ne dispose d'une faculté ressemblant à un « libre arbitre » : le groupe c'est le groupe et chez Aristote tout ce qui concerne la morale se voit inexorablement renvoyé à la science (pratique) de la politique. Selon ce point de vue, chaque fois qu'il est hors de son groupe l'individu est réduit à l'état de primate parlant ou peutêtre de viande, c'est-à-dire hors morale. La morale d'Aristote telle qu'il la décrit est

Op. cit., p. 135.

Aristote, Éthique de Nicomaque, texte, traduction, préface et notes par Jean Voilquin, p. 5.

Voir Jean-Pierre Vernant et Pierre Vidal-Naquet, Mythe et tragédie dans la Grèce ancienne, p. 52, note 20. Je dois à l'obligeance de Pierre Gravel de m'avoir signalé l'existence de ce texte. 
probablement plus proche de celle d'une société actuelle dite « primitive » que de celle de toute maisonnée ou groupe occidental, chrétien ou non.

Je dois supposer que ce genre d'inclusion de la morale dans le politique, ou une interprétation semblable de la morale tout aussi préchrétienne, prékantienne et dépourvue du concept de sujet, domine la plupart des interprétations de ce qui a été appelé plus haut la morale " classique », principalement parce qu'en dépit de leurs origines particulières si différentes elles partagent plus ou moins la même croyance et s'accordent étonnamment bien sur ce point crucial.

On reconnaîtra que cette convergence limitée de la morale aristotélicienne avec celles des sociétés préindustrielles ou primitives actuelles a relativement peu retenu l'attention de l'érudition traditionnelle portant sur la littérature classique du monde gréco-romain dans laquelle, au contraire, on remarque un manque d'intérêt manifeste pour l'ensemble des répercussions qu'entraîne le silence forcé d'Aristote sur la question du "libre arbitre » et du Sujet. Par exemple, on trouve dans l'ouvrage de E.R. Dodds ${ }^{1}$ portant surtout sur la Grèce archaïque, un chapitre 2 intitulé « De la civilisation de la honte à la civilisation de la culpabilité », mais encore l'auteur reconnaît-il ne faire qu'emprunter cette distinction à l'anthropologue Ruth Benedict ${ }^{2}$, et précise en outre : « Je ne me sers de ces termes que d'une manière descriptive, sans prendre à mon compte aucune théorie précise de la transformation des civilisations. " Alors qu'en fait le concept dont nous aurions besoin pour notre compte expliquerait non seulement le passage de la civilisation de la honte à une civilisation de la culpabilité mais en outre le passage de la culpabilité collective à la culpabilité personnelle.

On reconnaîtra également que le présent exposé, qui ne fait qu'esquisser cette question qui mériterait un traitement beaucoup plus élaboré, ne s'éloigne pourtant guère du point de vue adopté par l'érudition traditionnelle. On trouve, par exemple, sur le même passage d'Aristote cité plus haut (1094a) disant explicitement l'inclusion de la morale dans la politique le commentaire réprobateur suivant du traducteur Jean Voilquin : "Cette théorie, qui fait dépendre la morale de la politique, soulève de graves objections. L'attitude moderne est toute différente ${ }^{3}$. » Ce commentaire sans nuance résume à lui seul l'indifférence marquée sinon le refus sans appel du savant helléniste concernant ce qu'Aristote pouvait vouloir dire sur cette question pour nous de la plus haute importance. Sur le fond, Jean Voilquin a raison, l'attitude moderne est effectivement très différente, mais ce n'est pas une raison pour rabrouer Aristote, c'est cet écart même qui nous permet de comprendre que, contrairement à la nôtre, la morale d'Aristote est une morale de groupe, prékantienne; les "graves objections » dont le traducteur fait état ne traduisent que l'indignation de notre ethnocentrisme occidental.

Les Grecs et l'Irrationnel.

Ruth Benedict, The Chrysantemum and the Sword : Patterns of Japanese Culture, p. 222 et sv.

Aristote, op. cit., p. 511. 
Il en va de même quand je lis le commentaire d'Eric A. Havelock dans son Preface to Plato sur une possible anticipation platonicienne du concept de sujet dans La République (443c sv.) :

Lorsque nous lisons Platon il nous arrive de nous persuader qu'il n'y a pas de salut en dehors de la société, alors qu'en d'autres endroits il nous semble que c'est le royaume intérieur à l'homme qui pourvoit à tout. La République est équivoque [bifocal] dans l'importance qu'elle accorde à l'un et l'autre point de vue ${ }^{1}$.

Qu'est-ce qui est équivoque, ici, le texte de Platon ou bien la lecture qui en est faite? Qui saurait montrer chez Platon un "royaume intérieur à l'homme » capable, je ne dis pas de prévaloir mais seulement de se mesurer aux Lois et aux Gardiens ? Selon Robert Flacelière «La cité antique était, comme nous disons aujourd'hui, "totalitaire" ${ }^{2}$. » C'est là une réalité que l'admirable exposé sur la nature de la justice qu'est La République de Platon ne viendra jamais démentir.

Ce sont là autant de façons de reconnaître que la conception aristotélicienne de la morale m'apparaît très difficile sinon impossible à imaginer : comment peut-on se représenter la morale comme un simple chapitre dans un traité de politique ! Et je ne puis pas davantage, malheureusement, découvrir une interprétation moderne convaincante ou simplement plausible d'une telle doctrine rendant la morale tributaire de la politique ${ }^{3}$. Ce qui, réflexion faite, ne devrait pas étonner outre mesure si l'on interprète cette carence comme le résultat normal de l'ethnocentrisme occidental ordinaire méconnaissant le fait que la morale d'Aristote est une morale de groupe. On pourrait même prédire qu'une carence de cet ordre apparaîtra presque inévitablement dans notre discours et notre action, témoignant par là de notre relative incapacité à vraiment rendre justice à tout système moral quel qu'il soit à moins qu'il soit fortement imprégné de la doctrine chrétienne du sujet individuel, du «libre arbitre » et du salut d'une âme immortelle.

En dernière analyse, c'est toujours aux droits de l'homme qu'il faut revenir pour distinguer le plus nettement la morale fondée sur le sujet de la morale fondée sur le groupe ; dans une morale fondée sur le groupe, qu'en termes modernes on pourra dire "intégriste », la morale est au contraire assujettie à la politique comme l'affirme Aristote dans les premières pages de l'Éthique de Nicomaque.

Eric A. Havelock, Preface to Plato, p. 204.

Robert Flacelière, La Vie quotidienne en Grèce au siècle de Périclès, p. 238.

Il faut tout de même signaler, même si elle ne décrit que l'évolution de la pensée grecque jusqu'à Platon et sans la comparer à la pensée moderne, l'étude magistrale de F. M. Cornford, From religion to philosophy, montrant que l'origine de la morale chez les Grecs se situe dans le groupe et non pas dans l'individu. Voir en particulier p. 52-55. 
Les hommes du temps d'Aristote et de sa culture ne semblaient pas rêver de liberté ou de libération comme les Occidentaux sont portés à le faire, ils rêvaient au contraire d'être mieux intégrés au groupe, leur idéal était d'y être mieux retenus et intégrés, de mieux appartenir et participer à la vie communautaire et non pas de s'en affranchir. C'est pourquoi Socrate choisit la ciguë de préférence à l'exil qui lui était également offert mais qui paraissait beaucoup plus immoral à ses yeux ; choisir l'exil eût été l'équivalent d'accepter d'aller vivre avec des singes. Ceci ne déprécie en rien l'idéal pour lequel il est mort mais signifie simplement que pour un Grec de son temps la simple idée qu'un non-Athénien puisse avoir accès à l'ordre moral devait apparaître tout simplement ridicule ou absurde. Dans ces conditions l'ethnocentrisme et le racisme avant la lettre sont de rigueur, une composante de base de la moralité humaine. Le principal problème qui se pose à nous est qu'en dépit de toutes nos connaissances et de nos protestations à l'effet contraire, le racisme est tout aussi présent dans notre conduite morale qu'il est maintenant absent de notre théorie. Le prochain chapitre sera consacré à l'examen de la question raciste.

Pour le moment revenons aux Grecs. Pour eux la valeur et donc la morale est dans les choses elles-mêmes, dans ce que leur métaphysique appelle l'être, ce qui veut dire dans l'inégalité fondamentale (qu'on peut dire de nature divine) entre ces choses. Cette inégalité inclut évidemment les humains eux-mêmes : Platon estime que le travail manuel est en lui-même dégradant, Aristote pense que certains métiers sont si vils qu'ils mettent leurs artisans hors morale, les rendant radicalement incapables de tout accès à la vie morale. Cette inégalité est cosmique, divine, sociale, sexuelle et sanctionnée par le groupe avec toute la polysémie du mot sanction en français : c'est le groupe qui apporte sa sanction et donc qui avalise socialement l'inégalité entre les choses et entre les humains et c'est aussi le groupe qui sanctionne et punit quiconque tente d'y contrevenir.

Le Grec du temps d'Aristote ne semble pas pouvoir imaginer qu'une chose puisse être belle sans aussi être bonne et vraie. Il ne distingue pas l'excellence morale de l'harmonie et de l'esthétique. Dans cette vision morale l'excellence n'a rien à voir à ce qu'un homme veut ou ne veut pas, elle résulte au contraire d'une bonne disposition développée sous l'effet conjugué d'une bonne éducation, d'une naissance dans une famille de haut rang, de la richesse et de la fortune au sens de destin. Lorsqu'un homme est à ce point marqué à la fois par son hérédité et par son environnement, toute la question morale devient celle de savoir lesquelles de ses dispositions natives ou héritées l'individu doit développer par l'éducation et l'effort. C'est une question de jugement et de destinée, mais non pas de libre arbitre. Dans une telle perspective l'idée même de libre arbitre n'a aucun sens.

J'espère que ces détails apportent un nouvel éclairage sur les raisons qui ont fait écarter la plus vénérable et fréquemment débattue question de la morale occidentale, celle qui concerne le « libre arbitre », de notre définition de la morale humaine, parce qu'elle n'est manifestement pas universelle. En fait toute la 
question de savoir s'il existe ou non une telle chose qu'un « libre arbitre » perd pratiquement tout intérêt dès que l'on se rend compte que la Grèce classique, de même que bien d'autres sociétés passées et présentes, ne pouvait probablement même pas se représenter la nature même de ce qu'elle met en jeu. La question ellemême n'est donc pas une partie indispensable de la moralité humaine mais seulement de la morale occidentale, elle est ethnocentriste, elle est non seulement peu utile mais véritablement captieuse pour les fins d'une science de la morale.

Mais la chose a première vue la plus étonnante pour un moderne, presque incompréhensible, me semble-t-il, est l'absence de culpabilité individuelle ou personnelle dans la morale grecque ancienne. Comme dans de nombreuses sociétés préindustrielles actuelles la morale de la Grèce classique conçoit la faute sous forme d'une souillure affectant non pas un individu particulier mais un groupe (famille, ville, État). La purification de cette souillure pourra prendre diverses formes affectant ce groupe mais le Grec ne sait pas imaginer ou conçoit mal la faute, ni par conséquent le devoir ou le remords qui serait purement individuel.

Nous le voyons un peu mieux si nous comparons un élément de notre propre vision morale avec la sienne. Lorsque nous, Occidentaux modernes, sommes témoins d'un acte que nous estimons moralement très répréhensible ou répugnant, notre vision morale nous fait d'ordinaire éprouver deux sortes de sentiments : 1) En premier lieu nous sommes persuadés que l'auteur de l'action mauvaise est pourvu d'un intérieur, qu'il est ce que nous appelons un «sujet » et nous pensons que cet intérieur est dorénavant ou sera envahi sous peu par un sentiment personnel de faute ; 2) dans un deuxième temps nous sympathisons avec lui, nous prenons sur nous-mêmes une partie du regret et nous sentons un peu coupables avec lui. Nous psychologisons tout. Nous, j'entends les Occidentaux blancs scolarisés, en arrivons même à psychologiser les animaux dans ce que nous appelons les «droits des animaux » ce qui est un non-sens manifeste. Quoi qu'elle fasse notre morale campe Sa Majesté le sujet, Ego, au centre de la scène du monde et la psychologie devient progressivement l'équivalent fonctionnel d'une théologie bien particulière nous rendant dorénavant incapables d'irrespect envers lui, cet Ego en passe de devenir le seul dieu qui nous reste. L'athéisme devient une stratégie autodestructrice. Carl Jung disait en ce sens : « Notre vraie religion est un monothéisme de la conscience, nous sommes ses possédés ${ }^{1}$. » Quand Ego s'installe au centre de la scène du monde il est au cœur de ce désert qu'on appelle la modernité. Autrefois la modernité signifiait Newton et la révolution copernicienne de Kant, mais à notre époque certains de nos meilleurs théoriciens se disent post-modernistes alors que je les soupçonne de vouloir dire post-révolutionnaires.

Le Grec du temps d'Aristote semble n'avoir rien fait de tel. Il ne se reconnaissait pas un Ego capable d'un « libre arbitre » et demeurait sans doute peu doué pour la «crise d'identité ». Il semble avoir regardé l'auteur de la très

\footnotetext{
Cité par James Hillman, A Blue Fire : Selected Writings by James Hillman, p. 44.
} 
mauvaise action avec un sentiment proche du singulier mélange de détachement et de vague répugnance néanmoins accompagnée d'un vif sentiment d'infortune que nous éprouvons au récit d'une victime du sida. Le malheur qui afflige le sidéen nous semble si grand que nul mortel pourrait raisonnablement en être tenu responsable. C'est effectivement ce que disait le Grec de l'auteur de l'action très répréhensible : Il faut qu'un dieu l'ait aveuglé ! Le Grec du temps d'Aristote se libérait ainsi de ce qui, dans la suite de l'histoire, devait si durablement hanter la conscience morale de l'Occidental, toute la question de savoir a qui ou a quoi il convient d'imputer la responsabilité de la souffrance dans le monde, du mal et du « mal radical ». Le Grec retournait simplement le problème à l'envoyeur, là où se trouvait selon lui sa seule origine possible : les dieux. Génial !

Il semble extrêmement difficile à un Occidental moderne d'avoir accès au feeling que doit inspirer le fait d'appartenir à une morale de groupe. Personnellement je n'ai aucune hésitation à reconnaître que la chose m'est impossible. Le romancier américain Paul Auster, décrivant le sort fait à Job dans le récit biblique parle du shipwreck of the singular, "le naufrage de la singularité ". C'est l'une des meilleures formules résumant, pour le bénéfice de la conscience occidentale, l'essentiel de la morale basée sur le groupe. Encore que cette formulation soit anachronique, puisque la morale fondée sur l'individu est toute récente par rapport à la morale de groupe, mais cet anachronisme est lui-même caractéristique du point de vue occidental.

Nous pouvons à peine imaginer qu'il ait pu exister une époque dans le passé de l'Occident où le sujet n'existait pas, ni le «libre arbitre », ni par voie de conséquence l'objet lui-même tel que nous l'entendons dans le discours cohérent de la culture scientifique. La plupart des hommes devaient donc y être un peu comme des choses, sans liberté individuelle au sens moderne et par là unifiés dans la perfection ordonnée du groupe composant la cité grecque et qui est une hiérarchie.

\section{Inséparabilité du Sujet et de l'Objet}

On se rend compte de l'inséparabilité épistémologique du Sujet et de l'Objet et l'on comprend un peu mieux le caractère en pratique insoluble du projet scientifique d'une science qui serait hors toute valeur. D'un point de vue historique Sujet et Objet ont mis des siècles à apparaître, chacun de son côté. Le développement de l'idée de Sujet s'étend au plus tôt d'Augustin (vers 400) jusqu'au cogito de Descartes et surtout jusqu'à Kant (1781), alors qu'on situera celui du concept scientifique d'Objet à l'époque de Galilée-Lavoisier. Mais d'un point de vue épistémologique les deux concepts doivent appartenir à la même litée car il ne peut évidemment exister de Sujet libre réputé source de toute valeur morale qu'à la condition qu'il existe également un Objet moralement neutre, débarrassé de tout lien avec l'ancienne métaphysique et que le Sujet pourra alors investir à son gré de valeur. Dans ce développement les philosophes sont demeurés fascinés par la glorieuse naissance du Sujet et simultanément par celle de la démocratie, alors que 
de leur côté les scientifiques se sont surtout attachés à l'invention de l'Objet caractéristique de la science moderne.

C'est sans doute pourquoi les scientifiques sont condamnés à exiger que les sciences sociales deviennent un jour value-free mais c'est aussi pourquoi celles-ci ne peuvent pas vraiment l'être car le doublet Sujet-Objet de la science et de la morale modernes est radicalement insécable, il est au cœur de l'ethnocentrisme constitutif de la culture occidentale qui nous éloigne définitivement de la morale aristocratique grecque en même temps ou par le même effort qui nous a fait renoncer à sa métaphysique. Le doublet Sujet-Objet est aussi inséparable que peut l'être l'ensemble et son complément dans la théorie des ensembles. Incapables de renoncer au concept scientifique d'Objet nous sommes condamnés à nous considérer nous-mêmes comme de purs Sujets libres, libres de toute contrainte groupale et c'est précisément par l'impossibilité où nous nous mettons de penser l'influence du groupe sur notre morale que se forge le plus indissolublement l'ethnocentrisme moral de notre groupe.

Les Indiens ont un piège pour attraper des singes, fait d'une noix de coco évidée, fixée au sol et pourvue d'une poignée de riz déposée par un orifice juste assez grand pour permettre le passage de la main vide du singe et empêcher le retrait de sa main pleine de riz. Le singe ne lâche jamais prise et meurt bouffé par son rêve, plus exactement par les villageois relevant leurs pièges. C'est un peu la figure que nous présentons lorsque nous demandons que la science se libère des attaches tribales du sujet mais non pas de celles qui sont constitutives de son objet. Le piège est décrit par Robert M. Pirsig dans son Traité du zen et de l'entretien des motocyclettes ${ }^{1}$. Il dit aussi :

L'Église de la raison, comme toutes les institutions du système, ne se fonde pas sur la force individuelle mais sur les faiblesses de chacun. Ce que l'Église de la raison exige de chacun ce n'est pas l'aptitude mais plutôt l'inaptitude. Alors seulement s'estime-telle en mesure de se charger de votre éducation ${ }^{2}$.

Autrement dit, ce n'est que lorsque l'on consent à être illogique que l'on commence à être personnel mais l'Occidental peut toujours compter sur l'aide de son groupe pour le préserver, lui et son groupe, des embarras de ce genre d'accident.

C'est ce que signifie l'idée que la valeur est une contrainte imposée par le groupe. La valeur telle que nous la connaissons est le fruit de l'asservissement au pouvoir qu'elle rend possible. La faiblesse individuelle est la matière première de l'ethnocentrisme du groupe. La science sociale occidentale tire de toutes ses forces

Robert M. Pirsig, Robert, Traité du zen de l'entretien des motocyclettes, p. 264.

Op. cit., p. 332. Traduction modifiée. 
depuis plus d'un siècle sur la richesse immensément profitable de l'étroitesse d'esprit du scientisme.

On voit le genre de problème que cela pose à une approche qui se voudrait expérimentale et appliquée de la morale. Ce problème concerne les concepts fondamentaux et les objectifs qu'il convient de confier à une morale expérimentale modelée sur la science appliquée de la médecine. On peut s'attendre à trouver une métaphysique semblable, de notre point de vue occidental, à celle de la métaphysique classique grecque dans toute version existant à l'heure actuelle de la morale basée sur le groupe. Une telle métaphysique définit habituellement les valeurs non pas comme je l'ai fait ici comme des contraintes mais plutôt comme des échelons à l'intérieur d'une hiérarchie possédant un sommet et une base, enchâssées dans les choses elles-mêmes et censées avoir été " créées par Dieu » d'une façon ou d'une autre, jamais d'origine humaine. Ce qui devrait imposer de constantes difficultés à la compréhension et à l'interprétation d'un tel système de valeurs de type aristotélicien.

S'il est vrai que la morale humaine résulte de contraintes biologiques que j'ai appelées primaires, et que les valeurs affichées ne sont que les contraintes secondaires et ethnocentristes de la morale dite, il s'ensuit qu'une morale expérimentale et appliquée devrait demeurer attentive aux limites que son ethnocentrisme lui impose dans ses études et interventions occidentales et non occidentales.

\section{Le groupe comme tribu}

La plus importante de ces limites est le groupe. J'entends par groupe ce que Garrett Hardin a appelé une tribu :

Tout groupe de personnes qui se perçoit lui-même comme groupe distinct et qui est perçu de l'extérieur comme groupe distinct peut être appelé une tribu. Le groupe peut être une race, telle qu'on la définit ordinairement, mais ce n'est pas indispensable ; il peut aussi bien être une secte religieuse, un groupe politique ou professionnel. La caractéristique essentielle d'une tribu est son double standard de moralité - elle adopte un mode de comportement dans ses relations internes, et un autre pour ses relations externes ${ }^{1}$.

On trouve dans la Bible un exemple classique du double standard de moralité. «Tu n'exigeras de ton frère aucun intérêt ni pour de l'argent, ni pour de la nourriture, ni pour quoi que ce soit dont on prendrait de l'intérêt. De l'étranger tu pourras exiger de l'intérêt ${ }^{2}$. »

Hardin, Garrett, « Population Skeletons in the Environmental Closet ».

Deutéronome 23, 19-20. 
La formule de Hardin illustre assez bien la grande difficulté d'une morale appliquée dès que l'on tente de penser la vie morale en termes de groupe. Car en dépit de son apparence paradoxale ou qui peut sembler provocatrice la définition de Hardin reflète fidèlement la nature du groupe moral et ce d'au moins trois façons :

1. Malgré une formulation qui semble en faire une réalité psychologique (se percevoir et être perçu comme distinct), c'est l'application du double standard qui constitue le groupe, autrement dit son ethnocentrisme.

On pourrait s'objecter ici, puisqu'il s'agit ici d'êtres humains, à une description fondée sur le concept de groupe et reposant sur le concept en apparence dérisoire de tribu proposé par Hardin, au lieu de l'usage plus traditionnel reposant sur le concept de société. Une telle dérision est tout à fait exclue ici. Aux fins d'une science de la morale il est épistémologiquement plus acceptable, plus éclairant et la description est en terrain plus solide lorsque les sociétés humaines sont décrites en tant que groupes, ce qui est biologiquement justifié, plutôt qu'au moyen de l'usage rendu familier par les sciences sociales donnant toute l'importance au concept de société. Car il est dorénavant quasi impossible de dissocier le terme de société de l'acception plus que centenaire imposée par la sociologie, laquelle devrait par ailleurs adopter l'appellation plus modeste de sociologie humaine, mais ne le fait pas, et cet héritage historiquement limité à la vie sociale humaine transforme en pratique le concept en apparence neutre de société en un standard de perfection par rapport auquel tout autre groupe biologique fait figure de parent pauvre ou de forme larvaire ou déficiente. La société humaine n'a aucun titre à la revendication d'un tel standard, le standard est fourni par la vie. Certes, des différences existent en biologie entre une société et un groupe, mais notre principale préoccupation concerne la morale et par conséquent l'ethnocentrisme du groupe. Ce choix terminologique assure une compréhension plus adéquate des origines et des limitations de l'existence humaine.

En fait la formulation de Hardin est conforme au concept de groupe tel que défini en biologie sociale : "Tout ensemble d'organismes de même espèce qui perdure pendant un certain temps et dans lequel les membres interagissent manifestement davantage entre eux qu'avec d'autres organismes de la même espèce ${ }^{1}$. " Une partie de la difficulté vient de ce que chez les humains la liste des groupes auxquels chaque individu appartient (groupe d'âge, sexe, ethnie, profession, éducation, revenus, lieu de résidence, habitudes de consommation, pratique sportive, allégeance politique, etc.) n'est guère énumérable. Pourtant chacun de ces groupes est une «tribu » au sens de Hardin. La profession médicale est un groupe et une "tribu », tout comme le monde des affaires, le monde de l'éducation et la communauté scientifique.

Edward O. Wilson, Sociobiology : The New Synthesis, glossaire. 
2. Du point de vue de la morale dite en Occident, donc du point de vue des contraintes secondaires, l'idée de double standard de moralité semble être à sa face même un scandale et un affront. Pourtant d'un point de vue descriptif on peut sérieusement douter que la vie sociale que nous connaissons soit possible sans la pratique constante d'un double standard de moralité. Si nous ne pratiquions pas le double standard de moralité, serions-nous capables d'altruisme familial ? Pourrions-nous conserver de l'affection pour le membre de notre famille qui s'est mal conduit? Ne serions-nous pas radicalement incapables d'être racistes ? Sexistes ? Aurions-nous encore ces réseaux d'amis, de quartier, d'affaires, professionnels, où nous sommes assurés de « ne pas être n'importe qui » et qui, pour plusieurs d'entre nous, forment en pratique l'essentiel de notre vie sociale ? Aurions-nous autant besoin d'insister sur l'importance de l'indépendance du système judiciaire par rapport au politique ? Aurions-nous besoin d'une fonction publique dotée d'un statut particulier et de privilèges qui la mettent à l'abri des interventions politiques partisanes ? Seraitil encore nécessaire d'intégrer à l'intérieur même du Parlement un parti d'opposition dont la principale fonction est d'opposer sa façon de voir, y compris ses intérêts comme groupe, à la façon de voir et aux intérêts partisans du parti au pouvoir, institutionnalisant de ce fait la dispute dans les plus hautes sphères de l'État?

3. En fait, il semble bien que la formulation de Hardin n'apparaît scandaleuse que parce qu'elle semble inthéorisable et qu'elle offusque l'idée que nous avons de nous-mêmes et qui n'est que la valeur. Elle semble inthéorisable parce qu'elle contredit la contrainte secondaire d'égalité. Elle constitue un problème spécifique à l'Occident et c'est le genre de problème que n'aura jamais une société préindustrielle qui, bien loin d'affirmer l'idée de l'égalité humaine, proclame d'habitude au contraire haut et fort le double standard de moralité comme on peut voir dans Deutéronome 23,19. Pour nous, le double standard répond donc exactement à la définition donnée plus haut de l'immoralité : c'est « la mauvaise herbe » produite par la culture de l'égalité, c'est cette partie de la contrainte primaire qui n'est pas réalisée par la contrainte secondaire d'égalité et qui s'obstine à faire voir sa sale tête dans nos mœurs en dépit de tous nos efforts pour l'abolir car c'est précisément cette même contrainte secondaire d'égalité que nous révérons tant qui nous empêche de "voir » le double standard. Hardin a tout à fait raison, le double standard de moralité est au cœur de la vie du groupe.

La leçon me semble claire. À partir de 1776-1789 la morale dite en Occident a commencé à faire profiter chacun des membres de la société d'un grand nombre des privilèges auparavant réservés à la tribu dans laquelle jusque-là l'individu n'était rien par lui-même. À partir de ce moment historique de l'histoire de l'Occident l'individu s'est autonomisé relativement aux dépens du groupe, mais ce développement n'a pas aboli le tribalisme - je rappelle qu'il n'est pas en notre pouvoir d'abolir une contrainte primaire - il en a seulement humanisé les effets et 
gommé le concept. En rendant possible la multiplication des groupes à l'intérieur de l'ensemble social ce développement fait en sorte que chacun de nous, qui demeure tribaliste, l'est à moindres frais et voit s'accroître l'autonomie de la personne, mais ce développement entraîne un coût, une " taxe ", pour ainsi dire, levée par le tribalisme sur le bénéfice individuel.

\section{L'impôt tribal}

C'est dans cette voie qu'une morale expérimentale et appliquée devrait poursuivre la révolution démocratique de l'autonomie personnelle. Le premier, le plus grand et le plus permanent obstacle qui se dresse sur cette voie demeure le groupe et la méconnaissance de l'influence qu'il continue d'exercer. Il en sera probablement ainsi pour tout l'avenir prévisible, tant que nous serons des animaux sociaux. Cet obstacle est d'autant plus important pour nous Occidentaux que ce rôle joué par le groupe dans la formation de la valeur est généralement méconnu. Chaque fois que, sur un point donné, une société se met en frais d'accroître l'autonomie personnelle de l'individu, son action implique nécessairement une institution ou un groupe et chaque fois le développement qui en résulte n'est pas simple mais double. À chaque fois l'individu ne parvient à s'autonomiser réellement que parce que l'institution ou le groupe impliqué dans ce processus s'autonomie également. L'autonomie nouvellement acquise ne profite donc pas uniquement soit à l'individu soit au groupe comme aiment le croire les anarchistes et les libertaires, mais l'une au contraire passe par l'autre. C'est ce qu'exprime l'adage selon lequel « en politique le moyen dévore la fin »: l'accroissement de l'autonomie personnelle s'obtient au prix d'un « impôt » payé au tribalisme.

Exemple. Ivan Illich a fortement dénoncé certains effets pervers de l'autonomisation des institutions et des groupes, bien qu'il les appelle autrement. Les exemples qu'il donne du gâchis produit par les administrations et les institutions sont effectivement effarants et l'on peut reconnaître avec lui que l'école abrutit les enfants, les transports réputés rapides nous volent notre temps, l'administration de la santé dépossède les gens de leurs corps et les tue. Mais il interprète tous ces faits comme autant de " contre-productivités ", c'est le terme qu'il utilise ${ }^{1}$. C'est comme si l'on disait que les dividendes versés aux actionnaires ou le coût d'une police d'assurance protégeant une usine d'automobiles sont contreproductifs sous prétexte que cet argent ne sert pas à fabriquer des automobiles ! Les faits signalés par Illich sont vérifiables à faire peur mais son explication est aberrante et dans la longue tradition de l'idéalisme au grand cœur qui ne voit pas l'origine tribale mais seulement individualiste de la morale. Avant d'être au service des personnes la morale est au service du groupe et loin d'être contre-productifs ces faits représentent au contraire l'impôt tribal au bénéfice des intervenants du groupe, par exemple ceux de la profession médicale. Illich demeure au niveau des

Ivan Illich, Némésis médicale. L'Expropriation de la santé. Voir le titre du chapitre 3, p. 83. 
valeurs individuelles des contraintes secondaires et l'excellent accueil fait à ses ouvrages, malgré l'inefficacité manifeste des moyens qu'il propose pour remédier aux situations qu'il dénonce, montre à quel point nous en sommes encore là et nous raisonnons ordinairement comme lui.

Ces considérations sont nécessaires pour nous aider à mieux comprendre comment il se fait qu'en plus des raisons que l'on peut avoir d'attribuer, en général, un ancrage biologique à la morale, c'est en réalité le groupe et donc la forme locale de la société qui fournit la principale configuration de la morale locale. C'est dans le groupe que l'on découvre le principal moteur de l'ethnocentrisme et le double standard de moralité. En dernière analyse, c'est la puissante attraction exercée par le groupe qui doit être rendue responsable de l'échec plus que centenaire des sciences sociales à devenir effectivement value-free tel qu'elles se l'étaient promis dès le jour de leur naissance, aussi bien que, les contraintes secondaires trouvant leur origine dans le groupe, responsable de la faible probabilité qu'elles parviennent un jour à la neutralité promise. Il importe de garder à l'esprit que, bien que certains systèmes moraux, et non pas tous, ont choisi de rendre compte de leurs dispositions particulières en termes des droits de la personne, ces mêmes systèmes demeurent malgré tout fondamentalement enracinés dans la biologie et fondamentalement fondés sur le groupe, même s'ils parviennent dans une certaine mesure à dessaisir le groupe d'une partie du contrôle qu'il possède traditionnellement sur certaines composantes d'importance majeure du comportement humain, atténuant progressivement certains des traits les plus criants et les plus brutaux du contrôle social historiquement associé au tribalisme humain.

\section{Le groupe et l'inégalité}

La formule de Hardin du double standard de moralité apparaît donc jusqu'à un certain point descriptive de l'ethnocentrisme exercé aux dépens de toute personne extérieure à ce groupe ; c'est là que s'applique le double standard, entre ce qui est in et ce qui est out. Qu'en est-il cependant des relations à l'intérieur d'un groupe ? Dans l'Occident dont la vie politique est démocratique, en particulier, peut-on dire que la vie de groupe, à elle seule, favorise l'égalité ?

Le droit joue évidemment un rôle central dans l'égalité recherchée, mais le droit n'est pas toute la vie sociale, et quand on regarde l'ensemble dans lequel il s'insère la réponse à la question posée semble plutôt négative. On pourrait être porté à penser le contraire mais je crois que cela provient de notre habitude de penser par concepts et non par images; en ce sens c'est un artéfact de notre formation logique. Au niveau conceptuel ou logique on peut effectivement dire qu'une personne est simplement " différente » d'une autre, comme une femme est différente d'un homme par exemple, sans que cela implique pour autant l'existence d'une inégalité entre eux. Un concept est construit à l'aide d'autres concepts et subsiste par soi dans «l'espace mental », pour ainsi dire, différent de tout autre 
concept et apte à entrer à son tour dans l'élaboration d'une autre construction conceptuelle différente de toute autre.

Mais ce n'est là que le niveau du langage ou de la logique, ce n'est pas ainsi que les choses se passent dans un groupe réel composé d'organismes en interaction. Pour le voir il suffit de réinsérer le concept créé aux fins du discours cohérent dans la société ou le groupe qui l'avait inspiré au départ pour faire apparaître la règle pratique suivante : Montrez-moi une différence et je vous montrerai une inégalité. Tout organisme manifeste un comportement et tout comportement particulier se situe en un point quelconque d'une courbe statistique prédisant sa plus ou moins grande probabilité : l'ensemble est stochastique. Lorsqu'un comportement donné est fortement probable cela signifie qu'il est pratiqué par une majorité des membres du groupe et que l'organisme qui l'exhibe jouit des meilleures conditions de vie que le groupe puisse offrir. Si au contraire le comportement est moins probable, l'organisme impliqué fait partie d'une minorité par rapport à ce groupe et à ce trait et sa vie s'en trouvera affectée en conséquence. Le minoritaire, dans tout groupe et par rapport à n'importe quel trait, sera singularisé d'une façon ou d'une autre. Parfois, mais rarement, cette singularisation s'avère favorable et c'est le cas de l'individu dont on dit qu'il s'est trouvé au bon endroit au bon moment. Mais la plupart du temps, tout comme dans l'évolution elle-même, le comportement minoritaire ou exceptionnel est défavorable et l'individu concerné subit de ce fait la discrimination du reste du groupe, il peut s'attendre à être importuné, harcelé ou pourchassé de diverses façons qui varient selon de très nombreux facteurs comme la brutalité ou l'aménité de la culture locale, la nature des enjeux, la répartition locale du pouvoir et bien d'autres encore. Un Noir dans une société de Blancs peut s'attendre à subir ce genre de discrimination qui le traite comme un étranger dans son propre pays, mais cette règle s'applique aussi bien au nain ou au géant dans une société où la majorité des gens sont par définition de taille moyenne et où les sièges d'avion et de cinéma, les vêtements de confection et tous les produits imaginables sont conçus en fonction de la taille moyenne. La règle s'applique aussi à l'homosexuel minoritaire dans une société d'hétéros, au gaucher dans une société de droitiers, au Blanc qui tente de gagner sa vie dans une société de Mohawks, à l'albinos, à l'individu doté d'un quotient intellectuel hors moyenne. La règle s'applique à pratiquement toute conformation ou performance susceptible d'être mesurée ou perçue.

Il y a là une contrainte primaire particulière au groupe et qui reconstruit sans cesse l'inégalité à mesure que la démocratie tente et réussit en partie à la réduire. Le nom scientifique de cette règle est l'ostracisme, qui est l'un des plus puissant et indispensable facteur de vie sociale et de gestion de la vie en commun. Sans ostracisme la vie sociale serait manifestement beaucoup plus lourde à porter ; pour s'en convaincre il suffit d'essayer d'imaginer une société qui serait dépourvue de prisons ou de panonceaux affichant les mots « entrée interdite ». 
La contrainte imposée par la majorité s'exerce avant tout jugement moral qui serait porté sur les conséquences potentiellement fautives ou immorales de telle ou telle conduite. La courbe statistique des comportements prévisibles fait des individus des partants inégaux dans la lutte pour la vie; la reconnaissance subséquente de l'égalité des droits entre partants inégaux consacre souvent cette inégalité, quand elle ne l'aggrave pas. La courbe est une réalité de la vie sociale, une facette de la vie en groupe et de nombreux éléments de la vie sociale des animaux confirment son importance, notamment la prédation, la reproduction, le mimétisme, les dominances.

D'autres contraintes primaires produisent le même effet sur divers points. Certaines sont liées à l'histoire culturelle du groupe. En théorie, depuis la guerre de Sécession, le Noir américain est légalement l'égal du Blanc mais la libération de l'esclave qu'il fut n'en a pas automatiquement fait un homme libre ; on sait qu'à Rome ou ailleurs l'esclave libéré pouvait être considéré pendant encore longtemps exactement dans ces termes, comme un esclave libéré. D'autres contraintes sont liées au sexe. Dans toutes les sociétés humaines la femme est infériorisée à des degrés divers.

On conclura donc qu'en lui-même le groupe, tout groupe (d'organismes reproduits sexuellement) ne favorise pas davantage l'égalité entre ses membres qu'il ne favorise l'égalité entre ses membres et les autres individus qui n'en font pas partie.

L'ensemble de ce raisonnement ne doit pas être interprété comme un retour au « darwinisme social » du siècle dernier proclamant la victoire du fort sur le faible. C'est plutôt un retour à l'affirmation de G.E. Moore selon laquelle il est impossible de passer par voie logique d'une proposition à l'indicatif à une proposition à l'impératif, ou vice-versa, et c'est aussi un retour à la Troisième antinomie de Kant. L'inégalité est le résultat probable de la reproduction de la vie et du groupe, alors que la démocratie nous rapproche de ce que nous croyons que la vie humaine devrait être. C'est donc dire que ce raisonnement nous rappelle que l'égalité n'est pas une réalité et ne le sera peut-être jamais. La démocratie est un processus en marche, un chantier, une lutte pour la démocratie.

Ce qui signifie également entre autres choses que nous ne pouvons pas justifier l'adage populaire disant que le pouvoir corrompt. L'accès au pouvoir est possible au fol comme au sage. Le pouvoir n'est pas une chose mais un rapport, plus précisément c'est l'aboutissement d'un rapport d'inégalité. Quiconque affirme que le pouvoir corrompt montre qu'il entretient l'idée que la société humaine est composée de membres égaux et doit se contenter de vitupérer l'époque quand il s'avise qu'aucune société réelle n'a le bon goût de conforter son idée ; il exhale alors la sourde colère de l'individu qui se croit unique, qui croit n'être social qu'en vertu d'un «contrat social » librement consenti et qui souffre des affronts que la vie sociale quotidienne oppose à sa certitude bafouée. Nous devrions éviter de dire 
que le pouvoir corrompt parce que cette métaphore d'origine biologique sert alors de justification moralisatrice qui nous épargne la peine d'une description plus précise et exacte de la situation. Pour ma part, si je veux entendre des propos moralisateurs je puis aller à l'église ou au parlement, une taverne c'est encore mieux, mais si j'ouvre un journal je m'attends à trouver de l'information, c'est-àdire des choses que je ne sais pas. Je ne veux pas me faire dire que le pouvoir corrompt ou semblables sornettes. Si au moins l'on nous disait que les hommes de pouvoir sont en état de péché mortel cela nous informerait sur la religion du locuteur. La langue française, comme la plupart des langues je suppose, est très riche pour dire la concussion, le détournement de fonds, le trafic d'influence, l'abus de pouvoir, le népotisme, etc. Ce n'est que par le recours à de telles précautions que l'on peut songer à récolter le bénéfice de comparaisons construites à partir de la biologie sociale. Les singes rhésus, par exemple, excellent au partage népotistique du pouvoir et leurs sociétés ont parfois été appelées « aristocratiques » pour cette raison. D'autres comparaisons de ce genre, qui ont certes leurs limites, peuvent être utiles, mais nous devrions éviter l'usage de généralisations moralisatrices à base biologique dont la valeur informative est nulle, comme l'idée que le pouvoir corrompt.

Dans cette même veine terminologique, nous les philosophes, les spécialistes des sciences sociales ou journalistes devrions éviter le recours aux termes fondamentalisme ou intégrisme pour désigner certaines orientations religieuses, car ces termes n'apportent aucune information sinon qu'il s'agit de religion, ce qui relève du pléonasme puisque toute activité religieuse relève effectivement de la religion. Il en va autrement si la description, y compris à l'occasion celle des mouvements religieux occidentaux, réfère à un sens moral fondé sur le groupe, par opposition au sens moral fondé sur la personne et basé sur les droits de la personne.

\section{Le groupe et la démocratie}

La démocratie n'est donc pas l'égalité, quoi qu'en disent les textes et les constitutions, c'est un mode de redistribution de l'inégalité groupale : nous n'avons souvent de libertés individuelles que parce que notre groupe a réussi à contraindre les princes qui nous gouvernent à se faire concurrence entre eux. Jusqu'à un certain point, le meilleur moyen d'accroître l'autonomie de la personne est d'accroître la concurrence entre les institutions et entre les chefs; jusqu'à un certain point seulement parce que nous avons besoin de chefs, nous ne pouvons absolument pas vivre sans chefs, notre besoin d'avoir des chefs est beaucoup plus grand et pressant que notre besoin d'avoir de « bons » chefs, c'est-à-dire qui soient doués, prévoyants et ingénieux. Notre besoin de chefs est si grand qu'en temps de crise littéralement n'importe qui fait l'affaire.

Il est vrai que la démocratie instaure un État de droit mais la relation existant entre la morale et la loi n'est pas simple ni constante ou a sens unique. Parfois les 
deux instances concourent mais il leur arrive aussi de diverger comme on voit dans Antigone ou dans Le Marchand de Venise. D'un autre côté on peut aussi soutenir, particulièrement dans la tradition du rationalisme kantien, que la morale appartient à un domaine si fondamentalement différent de celui de la loi que les deux en deviennent incomparables. Bien que cette proposition ait quelque vérité son acceptation n'est pas suffisamment universelle pour être de grande utilité à toute personne se situant à l'extérieur - et osant remettre en question - la tradition rationaliste européenne ; comme dit plus haut, l'hétérogénéité de la morale et de la loi pourra sembler aller de soi à Paris ou à Berlin mais non pas à Téhéran, par exemple, où, au moins en théorie, la seule morale reconnue est précisément celle de la loi coranique : en Iran, la morale c'est la loi. Pendant de nombreux siècles une semblable interprétation légaliste de la morale a dominé la doctrine de l'Église catholique du droit canon et celle de la religion juive. On ne peut donc pas dire en toute généralité que la morale est radicalement différente de la loi.

On ne peut pas davantage généraliser en sens contraire et affirmer que la loi est une sorte de morale armée ou pourvue de dents, cette autre généralisation est insuffisamment descriptive car nous connaissons aussi des situations où c'est plutôt l'inverse qui est vrai et où c'est le sens moral qui prévaut contre la loi. Un exemple historique se trouve dans les procès de Nuremberg, en 1945 et 1946 après la Deuxième Guerre mondiale, où des gens furent accusés d'avoir violé des lois qui n'existaient pas au moment de la commission des actes qu'on leur reprochait : les « crimes contre l'humanité ». Je ne doute pas un seul instant que le sentiment d'horreur provoqué par l'énormité des crimes nazis et de l'Holocauste n'ait justifie la procédure et le châtiment qui s'ensuivit, mais il reste que dans ces conditions la justice qui prévaut devient la bonne à tout faire de la morale du vainqueur, celle d'un groupe contre un autre et hors toute norme juridique habituelle dans la société de droit. Des allégations semblables ont été portées contre l'action des forces d'occupation américaines au Japon à la même époque ${ }^{1}$.

Il reste tout de même qu'en démocratie, même lorsqu'elles viennent confirmer des contraintes secondaires de la morale, les lois sont adoptées après discussion publique par des représentants élus et remplaçables, et que ces lois demeurent sujettes à amendement. Souvent nous n'avons guère de moyen plus immédiat ou plus efficace que la rédaction des lois pour tenter d'affaiblir ou désarmer des mœurs tribales indésirables, des querelles domestiques mortelles ou des vendettas. Bien qu'elle prenne parfois des détours terriblement complexes et impersonnels la loi démocratique finit généralement par rendre la vie humaine un peu moins pénible et un peu plus supportable aux primates parlants; en vérité, l'un des principaux avantages de la loi démocratique est de faire en sorte que, pendant un certain temps, les primates parlants continuent à se parler.

\footnotetext{
Minear, Richard H. (1971) Victors' justice; the Tokyo War Crimes Trial.
} 
On doit donc se garder d'aller trop loin dans la confiance que nous, en Occident, aimons afficher pour l'égalité que la constitution démocratique assure à tous ses citoyens. Ici encore cette idée n'est pas fausse en elle-même mais elle est loin de mériter la primauté et l'universalité que les Occidentaux aiment lui attribuer. Comme toute pratique humaine ayant une dimension morale l'égalité affichée opère à la manière d'une contrainte secondaire qui, comme il a été rappelé à maintes reprises plus haut, ne prévaut que dans la mesure où elle réussit à appliquer effectivement quelque contrainte primaire propre à la biologie et ne contrevient pas aux dispositions d'autres contraintes primaires. Quiconque est le moindrement au courant des affaires humaines aura périodiquement l'occasion de méditer et la tentation d'adopter l'adage selon lequel les lois sont faites pour protéger les gens qui ont des choses à protéger contre les autres. Ce dicton a évidemment plus de chances d'être réputé immoral en démocratie que dans d'autres modes de gestion sociale comme les sociétés esclavagistes et les tyrannies, et cette différence est un résultat prévisible de l'équation

$$
(\mathrm{I})=(\mathrm{CP})-(\mathrm{CS})
$$

\section{Les trois éléments de la démocratie}

Il est souvent plus utile de se rappeler qu'un État fondé sur l'autorité de la loi n'est pas vraiment un état, c'est plutôt un processus en perpétuel besoin de renouvellement. Ce que nous aimons appeler les «droits » de tous ne sont rendus possibles que par la réduction des privilèges d'une minorité et cette réduction a des limites. Trois choses sont en effet indissociables et vont ordinairement de concert dans le développement historique des sociétés démocratiques : la morale à base personnelle (un «Sujet »), l'importance accordée à l'éducation et à la science (un monde peuplé d'«Objets »), et une forme ou une autre de capitalisme (défini comme la libre circulation des biens et de l'information) sont toujours présents dans la démocratie représentative à l'occidentale. Ces trois caractéristiques semblent constituer une unité fonctionnelle comme si, par-delà les particularités régionales et la diversité des appellations, elles relevaient toutes trois d'une même approche qui serait appliquée à une diversité de champs d'activité, dans le sens que lorsque l'une de ces trois composantes est forte les deux autres ont aussi tendance à être fortes, et lorsque l'une des trois est faible les deux autres ont aussi tendance à être faibles. Cette généralisation empirique souffre d'évidentes exceptions, comme en Inde dont l'économie essentiellement agricole et la morale traditionnelle des castes laissent peu de place à la science et à une morale à base personnelle, et qui possède pourtant une vie démocratique et une presse remarquablement libre depuis 1949. Mais le phénomène signalé prévaut tout de même suffisamment pour que, chaque fois qu'on trouve une société où la morale est à base personnelle, on puisse prédire l'existence d'une vie politique démocratique et une importance proportionnelle accordée à l'éducation, à la science et au capitalisme. À l'inverse, il est possible sur cette même base de mieux comprendre les nombreux échecs rencontrés dans diverses tentatives de démocratisation au cours de ce dernier quart 
de siècle, notamment en Europe de l'Est, au Moyen-Orient et dans les Antilles. On peut en effet prédire que là où la morale personnelle et/ou l'éducation et la science et l'économie de marché sont relativement faibles ou absentes, les institutions démocratiques ont peu de chances de s'instaurer et de prospérer.

Une morale expérimentale appliquée s'efforcerait ainsi d'identifier le rôle du groupe et des contraintes primaires dans les dysfonctionnalités observées. Elle saurait reconnaître à quel point il est « normal », je veux dire prévisible, que la médecine, comme institution et comme groupe, s'autonomise relativement et se mette au service des médecins en même temps qu'elle est au service de ses patients; que l'école, le syndicat, la magistrature, le parti politique et la communauté scientifique ont de même tendance à se mettre au service des intérêts de groupe de leurs praticiens. Toutes ces dysfonctionnalités apparentes peuvent être extrêmement précieuses, la réalité n'ayant jamais tort, elles sont l'indice de l'existence de contraintes primaires méconnues ou sous-évaluées dont il pourrait être possible, après recherche expérimentale, de réduire l'effet indésirable par le recours à de nouvelles contraintes secondaires plus humanistes occasionnant un accroissement de la concurrence entre les groupes ou entre les leaders. 


\section{Chapitre 4}

\section{La question du racisme}

\section{$\underline{\text { Retour à la table des matières }}$}

Le racisme est l'un des plus importants et difficiles problèmes émergeant de façon plus ou moins permanente à la surface de la conscience morale occidentale contemporaine. Cette importance, jointe au fait que ce problème fut utilisé plusieurs fois dans les pages qui précèdent comme exemple d'immoralité couramment rencontrée dans l'Occident démocratique, justifie un examen qui tente d'en dégager les éléments essentiels.

\section{Trois principes de la recherche}

Les textes publiés sur ce sujet sont relativement nombreux, les opinions qu'ils diffusent sont partagées, l'ensemble de cette question demeure fortement matière à controverse ; autant de facteurs qui portent à la modestie et nous empêchent de prétendre vider la question dans les lignes qui suivent. Trois principes généraux seront mis en œuvre pour aborder cette question.

1. Le problème du racisme sera examiné du point de vue de la biologie évolutionniste afin d'en identifier les racines ou les origines. On considérera que ce problème est universel, c'est-à-dire qu'il se manifeste dans toutes les sociétés et aucune société humaine connue n'en est exempte ; il est endémique c'est-à-dire permanent et de tout temps, quoi qu'il puisse présenter des accès ou des alternances de hauts et de bas.

On ne peut pas supposer pouvoir jamais s'en débarrasser complètement, il faut au contraire prévoir que les conditions qui l'ont fait naître dans le passé peuvent le ressusciter dans l'avenir dès que les circonstances changent ou que l'attention qu'on lui porte se relâche. Enfin je ne prétends pas que l'examen qui va suivre présente la totalité du phénomène raciste; l'entreprise vise plutôt à tenter d'identifier et comprendre ce que j'appellerai le racisme de base, les composantes indispensables à son existence et perpétuation. 
2. Le fait de mettre à contribution la théorie de l'évolution pour comprendre l'origine du problème a pour première conséquence d'écarter, dès le départ, l'idée qu'il puisse être de nature psychologique ou cognitive. On supposera donc que pour être raciste il n'est pas nécessaire de savoir ou même d'admettre qu'on l'est ; on supposera de même que le racisme n'est probablement pas sous le contrôle de la volonté, dans le sens qu'on peut l'être de façon tout à fait involontaire, on peut même être persuadé que ce comportement est répréhensible et vouloir cesser de le manifester tout en continuant néanmoins à éprouver des sentiments, entretenir des idées et agir de façon raciste.

3. Ce qui ne veut évidemment pas dire que la compréhension et la volonté d'intervention sont sans intérêt dans le traitement de ce problème, mais simplement qu'il convient de séparer l'identification du problème de l'ensemble des actions qui peuvent être entreprises par la suite pour tenter d'en réduire l'incidence.

L'approche veut être pratique. La question est évoquée parce que le problème constitue une des plus grandes tares qui déshonorent en permanence la société occidentale en dépit des grands principes énoncés dans ses déclarations et chartes des droits. La science, dans la mesure où elle peut nous apporter un éclairage utile, sera mise à contribution, mais le présent examen de cette question, après un bref rappel de nos connaissances objectives, cherche plutôt à fournir des moyens efficaces d'agir sur la situation. Le racisme n'est pas ou ne devrait pas être traité comme la température dont Mark Twain disait que tout le monde en parle mais personne n'y fait rien. Il s'agit essentiellement d'un premier pas vers la réinsertion du racisme dans l'ensemble de nos connaissances et une invite à la recherche expérimentale sur le comportement et sur la modification du comportement.

\section{La situation a changé depuis trente ans}

Deux auteurs, John F. Dovidio et Samuel L. Gaertner, étudiant l'état de la discrimination raciale aux États-Unis, font état de tendances qui portent à croire que la situation a nettement évolué depuis le début des années 1960 et le mouvement des Civil Rights. Malgré l'ampleur du chemin qui reste à parcourir, la vie quotidienne et l'importance des émeutes raciales qui viennent périodiquement rappeler aux Américains l'existence de la discrimination raciale, il reste que les progrès sont appréciables. Mais ce serait une erreur de pavoiser, disent ces auteurs :

Bien que les études que nous avons citées montrent l'existence de tendances à l'égalitarisme dans les attitudes raciales, les stéréotypes et les portraits présentés par les médias, il reste que les chiffres cités surestiment l'ampleur du changement par 
lequel l'Amérique blanche pourrait avoir réellement renoncé à ses griefs à l'égard des Noirs et des autres minorités ${ }^{1}$.

Avec le temps, le racisme américain change de forme, les injustices les plus criantes sont en voie de disparition mais plusieurs des changements intervenus pourraient affecter davantage les apparences que la nature même du phénomène.

\section{Qu'est-ce que le « racisme»?}

Nous commencerons par examiner l'idée de "racisme » qui pose problème, et d'autres qu'on lui associe, comme «préjugé » ou «discrimination ». En premier lieu, certains chercheurs dans ce domaine, sociologues ou psychologues effectuant des études sur le racisme par voie de sondage d'opinion tels Richard A. Apostle et al. ${ }^{2}$ qui auraient souhaité pouvoir catégoriser leurs informateurs selon qu'ils se montraient « racialement préjugés » ou au contraire "non racialement préjugés » ont finalement renoncé en cours d'enquête à utiliser des termes comme " préjugé » ou « racisme » parce qu'ils estiment que ces mots véhiculent des connotations de type subjectif ou des valeurs morales qui les rendent peu propres à une recherche de type scientifique.

Tous les chercheurs ne sont pas de cet avis mais on peut comprendre ces hésitations parce que même en biologie (que ces sociologues ou psychologues du racisme n'interrogent pas à ma connaissance), le concept de race n'est pas fixe ou n'est pas clair. Comme le dit Frederick S. Hulse :

Une espèce est un ensemble génétiquement clos alors qu'une race ne l'est pas. En conséquence, une espèce est " pure » alors que toutes les races sont et ont toujours été « impures », c'est-à-dire, dotées de gènes venant de diverses autres races ${ }^{3}$.

Une espèce est une unité évolutive fermée dans le sens qu'elle n'échange pas de gènes avec d'autres espèces. Il n'en va pas de même pour une race. L'idée qu'une race est toujours "impure » est contre-intuitive, surtout lorsqu'on songe aux arguments fréquemment allégués par les racistes désireux de « conserver la pureté de la race » : c'est une caractéristique courante de l'attitude raciste que de raisonner comme si leur groupe particulier était en fait leur espèce ; et l'inverse est aussi vrai, les racistes ont aussi tendance à considérer que les autres groupes ethniques sont composés de non ou de sous-humains. Nous constatons encore une fois l'influence déterminante de l'ethnocentrisme et l'omniprésence du groupe comme facteur décisif non seulement du comportement social mais jusque dans la formation de la

1 John F. Dovidio et Samuel L. Gaertner, « Prejudice, Discrimination, and Racism : Historical Trends and Contemporary Approaches ».

2 Richard A. Apostle, Charles Y Glock, Thomas Piazza, Marijean Svelze, The Anatomy of Racial Attitudes, p. 36, note.

3 Frederick S. Hulse, « Social Behavior and Human Diversity », p. 77. 
pensée «spontanée » ou non critique. On pourrait être porté à croire que la dénonciation de cette fausseté qui consiste à présenter la race comme "pure » pourrait servir d'argument éclairant et convaincant dans la lutte politique contre le racisme. Néanmoins, il y des raisons de croire que les bénéfices à tirer de cette information sont limités ou de faible valeur car d'un point de vue scientifique le problème lui-même est plus compliqué encore.

Hulse explique qu'il n'y a qu'une espèce humaine alors qu'il y a plusieurs races humaines et que celles-ci changent avec le temps. Bien que nous puissions être intuitivement convaincus de pouvoir reconnaître à vue de nombreuses races humaines différentes, le concept de race n'est pas biologiquement clair ou dépourvu d'ambiguïté parce que les races ne sont pas simplement des ensembles d'individus présentant des ressemblances visibles communes; il n'y a pas de race d'albinos, pourtant les traits albinos peuvent apparaître dans toutes les races. C'est plus compliqué, dit Hulse.

Donc, même en biologie le concept de race n'est pas clair. Cette difficulté est fondamentale et l'on ne connaît pas de moyen de la surmonter en dépit des nombreuses tentatives faites en ce sens pour le raffiner et lui assigner clairement des limites au moyen de critères de plus en plus sophistiqués. Comme le dit Theodosius Dobzhansky, il n'y a pas de solution au problème du flou du concept :

On peut tenter de sortir de cette difficulté en identifiant plusieurs races intermédiaires ; ou encore l'on peut supposer que les races étaient nettement séparées dans le passé et qu'elles se sont par la suite mélangées à l'occasion de croisements. Cela n'est d'aucun secours. Plus on distingue de races différentes, plus leurs frontières deviennent floues. Et la difficulté n'est en rien limitée à l'être humain, elle affecte tout autant un grand nombre d'espèces dans lesquelles on ne peut pas l'imputer à des croisements antérieurs ${ }^{1}$.

On doit donc conclure que, bien que le terme de race soit significatif au niveau intuitif et que nous croyons savoir ce que nous disons en parlant d'un être humain de la communauté scandinave, d'un Juif ou d'un Mohawk, le terme se prête mal à l'utilisation rigoureuse que l'on exige du discours scientifique. Ce qui ne nous empêchera pas de parler de race et de racisme ici, car même si ces termes n'ont pas d'usage en science proprement dite, l'importance très grande qu'ils ont acquise en politique et par conséquent pour la philosophie n'est en rien affectée par cette limitation : il arrive que les humains parlent sans attendre que les scientifiques leur en donnent la permission.

Theodosius Dobzhansky, « Race Equality », p. 14. 


\section{L'histoire évolutive des primates}

D'autres éléments de l'histoire évolutive humaine rendent la question raciale difficile à cerner. Les estimés courants situent l'origine de l'espèce humaine à environ 250000 ans ${ }^{1}$. Il y a des raisons de croire que jusqu'à récemment, environ 5000 ans, nos ancêtres vivaient dans des groupes relativement petits. Et ce qui est surtout important par rapport à notre problème, il semble que les différentes races humaines vivaient dans des territoires différents, on dit alors allopatriques, tout comme certaines espèces animales d'aujourd'hui sont allopatriques. En conséquence on peut aussi penser que ces protohumains et humains vivaient dans des groupes ethniquement homogènes. Ce n'est donc que récemment dans l'histoire de l'humanité que plusieurs races sont devenues sympatriques, c'est-à-dire qu'elles se rencontrent sur une base régulière dans un territoire commun comme les Blancs, les Noirs, les Chinois, les Pakistanais et les Mohawks vivant tous à Toronto.

D'un point de vue évolutionniste il est possible que ce passé de notre espèce ait pu nous préparer à vivre de façon harmonieuse à l'intérieur d'une structure sociale comprenant un petit nombre de personnes, à peine quelques centaines ou quelques milliers d'individus, mais non pas dans un État moderne composé de plusieurs millions d'individus regroupant de nombreuses ethnies différentes. L'État moderne est donc une création spécifiquement humaine sans antécédent dans l'évolution de la vie primate. Et cela est surtout vrai de la société de droit à l'occidentale proclamant l'égalité de tous les humains et qui n'a guère que deux cents ans. On doit penser que, évolutivement, le passé culturel de notre espèce nous a bien préparés à vivre dans de toutes petites sociétés homogènes ${ }^{2}$, xénophobes et prospérant dans le cadre d'une structure de pouvoir népotiste. Il n'est donc pas tellement étonnant que nous éprouvions tant de difficultés, que nous rencontrions des problèmes plus ou moins permanents lorsque l'État moderne nous rassemble par millions, par dizaines ou centaines de millions dans des sociétés multiethniques et prétendument égalitaires.

Il peut aussi être utile de se rappeler à ce moment-ci l'échec constant et inexpliqué du " petit groupe » de la psychologie sociale, tel le «T-Group », au niveau du macrosystème ou niveau politique des sociétés occidentales. Au cours des quarante dernières années, la technique du petit groupe inventé par des psychologues a fait merveille en s'appuyant exclusivement sur la coopération de ses membres dans pratiquement toutes les composantes du complexe industrieladministratif : conseils d'administration, groupes de thérapie, conflits de travail, centres de recherche, et beaucoup d'autres. Son étonnante incapacité à produire des résultats analogues au niveau politique, demeurée inexpliquée, pourrait être interprétée comme une indication qu'il existe des limites inhérentes à la

Stephen Jay Gould, Wonderful Life. The Burgess Shale and the Nature of History, p. 46.

Voir Ben W. Ike, «Man's Limited Sympathy as a Consequence of his Evolution in Small Kin Groups ». 
coopération chez les primates, et que de telles limites, s'il en est, n'ont pas été identifiées comme telles par les psychosociologues qui en subissent les inconvénients mais demeurent si loin d'en avoir identifié l'origine qu'ils ont plutôt tendance à nier l'existence même de cette limite imposée à une méthode de recherche et d'intervention par ailleurs si féconde ${ }^{1}$.

Ce genre de réexamen de l'ensemble des composantes de l'histoire de la nature humaine pourrait nous aider à comprendre un peu mieux comment l'État moderne en est venu à constituer un macrosystème social qui engendre des problèmes que nous sommes évolutivement inhabiles à gérer et à résoudre. Les moyens habituels non agressifs dont nous disposons dans nos microsystèmes sociaux, j'entends les moyens légaux, religieux, économiques, psychologiques qui ont fait leurs preuves au niveau du petit groupe et de la vie de village, ne sont manifestement pas adéquats pour nous permettre de faire face aux problèmes posés par les macrosystèmes que sont devenus l'État moderne et la très grande ville. Objectivement et dans l'histoire c'est à la guerre que l'humanité a eu le plus fréquemment recours pour gérer le problème du racisme endémique. Dans ces conditions on peut s'attendre à ce que cette relative impréparation de notre conditionnement moral historique demeure pour longtemps encore le lot de l'humanité.

\section{La nouveauté de la morale bourgeoise}

Karl Marx avait remarqué que la révolution bourgeoise ou révolution industrielle qui, depuis le tournant du XVIII ${ }^{\mathrm{e}}$ et du XIX ${ }^{\mathrm{e}}$ siècles porte l'essentiel de la culture et de la civilisation occidentales est la première grande domination humaine à imposer les principaux éléments de sa propre culture et de sa morale aux divers peuples qu'elle conquiert et soumet. Un général romain faisant une conquête en Mésopotamie ou en Germanie accordait moins d'importance à la conversion religieuse et morale des peuples conquis qu'à leur soumission aux lois et à la présence militaire de la Rome impériale. Il pouvait, selon les circonstances, soit les confier à un administrateur prélevant un tribut, soit réduire les femmes et les enfants à l'esclavage après avoir mis à mort tous les adultes mâles, soit encore les massacrer tous. L’hégémonie propre à la révolution industrielle est différente, elle repose moins lourdement sur la force militaire et davantage sur l'économie et sur la bonne volonté des vaincus à contribuer au succès de cette économie. Cette nouvelle forme de pouvoir rend souhaitable la conversion religieuse et morale des vaincus à l'éthique du travail industriel, à la religion chrétienne et à la théorie de l'égalité entre les êtres humains.

On est d'autant plus étonné de voir la rapidité avec laquelle le leader mondial incontesté de cette nouvelle ère de l'humanité, les États-Unis d'Amérique, a

Pour une analyse plus détaillée de cet échec relatif du Petit Groupe, voir Claude Lagadec, « Un point de vue moral : Le probable et l'improbable ». 
découvert le racisme. Pendant un siècle, de 1776 à 1865, les USA ont été égalitaristes, au double sens d'anti-aristocrates et d'anticolonialistes tout en demeurant néanmoins esclavagistes : tous les hommes étaient égaux mais les Noirs n'étaient pas des hommes. Après la guerre de Sécession les Noirs deviennent des hommes et des femmes, mais il suffira de quelques dizaines d'années pour que la culture esclavagiste soit remplacée par la culture raciste. Ce développement historique est tout à fait extraordinaire et à ce jour difficile à comprendre, à la fois parce qu'il était théoriquement inattendu puisque l'esclavagisme a été rendu illégal en bonne partie au nom des valeurs typiquement américaines d'égalité et que les Quakers y ont joué un rôle de pionniers, et aussi parce que le racisme ne pouvait naître ni du hasard ni d'aucune volonté identifiable, délibérée: comment un développement si horrible peut-il avoir lieu quand personne n'en veut ? Pour trouver réponse à cette question il faudrait supposer, durant cette courte période séparant la fin de l'esclavage du début de la culture raciste, moins d'un demi-siècle, l'existence de puissantes forces dont la nature reste à identifier et capables de produire un résultat aussi étonnant. Un siècle s'écoulera, de 1865 à 1959, avant que le Mouvement des droits civiques commence à faire reculer le racisme américain de façon un peu importante. Comment expliquer l'apparition d'un développement historique si manifestement contraire à la volonté des abolitionnistes américains et au credo américain?

\section{Le principe de xénophobie}

Pour toutes ces raisons, (le manque de clarté du concept de race, l'histoire évolutive des primates, la nouveauté de la révolution bourgeoise et l'incompréhensible remplacement de l'esclavagisme par le racisme, en plus de l'apparition très récente, déjà mentionnée, des termes "racisme » et « xénophobie »), je propose de considérer que la force à l'œuvre dans ce développement historique est la xénophobie animale. Le racisme humain est la forme spécifiquement humaine de la xénophobie. La xénophobie animale est une contrainte primaire. Vu sous cet angle, le racisme humain apparaît comme la forme spécifiquement humaine de la xénophobie animale. La xénophobie animale constitue alors le racisme humain de base.

En biologie sociale la xénophobie est considérée comme l'une des causes immédiates de l'agression. Le problème est posé dans les termes suivants par Edward O. Wilson :

L'apparition d'un étranger, chez les animaux, est le plus fort stimulus de l'agressivité, particulièrement celle de l'intrus territorial. Ce principe de xénophobie a été vérifié chez pratiquement tous les groupes d'animaux manifestant les formes les plus développées d'organisation sociale. Les lions mâles, d'ordinaire les adultes les plus léthargiques de la troupe, sont brusquement mis en alerte à la vue de mâles étrangers et se lancent dans une série de rugissements furieux. Rien dans la routine quotidienne de la vie sociale d'une colonie de fourmis, même aux moments de la tension la plus grande, n'excite autant le groupe que l'introduction de quelques ouvrières étrangères. 
Le principe s'étend aux primates. Southwick ${ }^{1}$ a mené une série d'expériences contrôlées sur des rhésus en captivité afin d'évaluer l'importance relative de plusieurs grands facteurs d'agressivité. Le manque de nourriture entraîne en pratique une réduction des interactions d'agressivité-soumission, car les animaux réduisent alors tous les échanges sociaux et commencent à consacrer plus de temps à une lente et laborieuse exploration de l'enclos. La surpopulation entraîne un accroissement d'un peu moins du double des interactions agressives. L'introduction de rhésus étrangers au groupe, cependant, entraîne un accroissement de quatre à dix fois plus grand de telles interactions. Cette étude quantifie de façon plus précise les faits ordinairement constatés dans la nature. Le taux d'agressivité manifesté lors de la rencontre de deux groupes de rhésus, ou lorsqu'un étranger tente de s'immiscer dans un groupe, excède de loin ce que l'on peut voir dans les troupes traversant les épisodes difficiles de leur vie quotidienne ${ }^{2}$.

D'un point de vue intuitif (que seule une recherche expérimentale pourrait venir confirmer), la xénophobie semble jouer un rôle pacificateur à l'intérieur du groupe aux dépens de tout ce qui est extérieur au groupe. Dans la plupart des sociétés animales de quelque complexité l'égalité des membres ou l'absence de dominance et de stratification sociale s'accompagne de conflits. Les animaux nouvellement assemblés s'agressent, consomment peu, leur santé est médiocre et leur reproduction faible ou inexistante. L'apparition de dominances (qui n'est pas la même chose que le statut social), parfois l'établissement de relations dyadiques dans lesquelles un organisme donné établit et conserve par la suite sa préséance sur un autre organisme, puis la naissance d'une hiérarchie sociale complète dans laquelle chaque organisme se voit attribuer un rang, un statut qui lui impose la préséance de quelques organismes et lui confère la préséance sur quelques autres, en même temps que se développe une hostilité ouverte qui peut être sans merci à l'égard de tout étranger au groupe, tout cela s'accompagne d'une paix sociale croissante au point, disent les spécialistes, que l'observateur non averti est porté à croire que cette société est fondamentalement dépourvue de toute forme d'agressivité. Ce développement : hiérarchie-xénophobie-paix sociale croissante, s'accompagne également d'une augmentation de la consommation de nourriture de chaque animal, d'une amélioration de la santé et de la vigueur de chacun, et d'une optimisation de la reproduction.

Autrement dit, en biologie sociale la xénophobie est la recette de la paix sociale, particulièrement dans les sociétés marquées par un leadership fort. En ce qui nous concerne, nous, les humains, nous sommes tentés de croire que c'est l'hostilité xénophobe individuelle qui déclenche la violence si fréquemment constatée dans les conflits raciaux entre les humains. Un examen plus attentif peut montrer que cette interprétation est une méprise. En général, l'égalité entre les

1 C.H. Southwick, " Aggressive behaviour of rhesus monkeys in natural and captive groups »; et "An experimental study of intragroup agonistic behavior in rhesus monkeys (Macaca mulatta)».

2 Edward O. Wilson, Sociobiology: The New Synthesis, p. 249. 
humains et entre les races et en l'absence d'un leadership politique fort (y compris le totalitarisme), favorise le développement des troubles sociaux. Nous savons que la démocratie est un système social avancé, qu'elle coûte cher et qu'elle est difficile à maintenir ; il est raisonnable de penser que le risque de troubles raciaux constitue une partie de ce coût encouru par l'égalitarisme démocratique.

De plus, c'est la recherche d'égalité qui est spécifiquement humaine et probablement sans véritable précédent dans le monde animal sexué, et non pas la xénophobie qui est une condition habituelle de la vie sociale. Sans un minimum de xénophobie prenant au moins les formes les plus modérées de l'ostracisme, il ne semble pas y avoir de société complexe possible, tout au moins pour les êtres sexués. En biologie la xénophobie est la première composante de l'agressivité et donc de la hiérarchie et de l'inégalité sociales, elle est un facteur de paix dans le groupe, et plus la dominance et le leadership sont forts plus grande est la paix sociale. Ce lien entre la xénophobie et la paix sociale semble très général, tout au moins en biologie sociale. Qu'en est-il en réalité ? Si la science morale expérimentale dont je propose la création devait éventuellement voir le jour, c'est là la toute première étude empirique que je souhaiterais lui voir entreprendre, telle est la question qu'elle devrait d'abord s'attacher à résoudre.

Nous sommes maintenant en état de poser la question principale : qu'est-ce, en vérité, que le racisme humain ? Peut-on éviter de voir et d'interpréter le racisme humain comme je l'ai fait ici, c'est-à-dire comme une simple forme, finalement banale dans sa prévisibilité, de la xénophobie animale? Vouloir l'interpréter autrement, vouloir continuer à croire que le racisme humain est spécifique à notre espèce et qu'il doit donc être interprète en dehors de toute référence à la nature biologique de notre existence, cela ne devrait-il pas être considéré foolishly parochial, pour reprendre l'expression de John Maynard Smith, autrement dit stupidement chauvin ? Il y a des domaines dans lesquels les humains sont uniques et leur conduite sans exemple dans le monde des autres vivants, mais pas en matière de xénophobie où leur comportement est au contraire trop prévisible et conforme à la règle qui prévaut chez les autres primates.

Si l'on adoptait cette interprétation faisant du racisme la version spécifiquement humaine de la xénophobie animale, il faudrait constamment se rappeler la nature possiblement pacificatrice et fondamentalement coopérative de la xénophobie : elle prend la forme d'une intervention concertée visant à renforcer la coopération sociale face à un danger qui menace l'ordre social tel que perçu. Nous savons trop bien à quel point les incidents raciaux particuliers peuvent engendrer la violence dans les sociétés humaines et qu'une telle violence peut parfois prendre en très peu de temps des proportions terrifiantes, pourtant même dans ces conditions le problème demeure fondamentalement le même. La xénophobie humaine se manifeste comme la prise à partie de plusieurs membres de la société contre $\mathrm{X}, \mathrm{Y}$ ou $\mathrm{Z}$ parce que, justifiés ou non, ces membres croient que l'ordre social de leur société est en danger, que la situation menace leur existence collective telle qu'ils 
la perçoivent et c'est pourquoi ces membres se déchaînent. La première tâche de la recherche qui s'impose serait de déterminer précisément la nature du problème déclencheur de l'attaque contre $\mathrm{X}, \mathrm{Y}$ ou $\mathrm{Z}$ : qu'y avait-il dans la société qui fût de nature à déterminer les membres à passer à l'attaque ? C'est la toute première question que les enquêteurs devraient se poser, en faisant la part qui lui convient à la dimension possiblement épidémiologique du phénomène. La réponse peut alors prendre la forme d'un diagnostic, forme telle qu'elle rende possible un traitement ayant quelque chance de succès.

Ceci n'est évidemment que de la théorie, mais aucune recherche expérimentale n'est possible sans théorie et la plus explicite possible. Dans la vie animale la xénophobie semble être une solution opérante; si nous voulons l'empêcher d'apparaître ou de prendre ses formes les plus brutales dans les sociétés humaines, si nous croyons que pour les humains elle est un problème plutôt qu'une solution, alors nous devons commencer par établir un diagnostic susceptible de mener à une solution plus humaine.

\section{Que faire?}

Ma première et seule réponse à la question «Que faire ? ", se résume à une recherche expérimentale basée sur la théorie évolutionniste. Je ne veux évidemment pas laisser entendre qu'aucune recherche n'a été faite sur le racisme. Nous savons au contraire qu'un grand nombre de travaux ont été et sont encore poursuivis, notamment au moyen de sondages d'opinion, pour contrer le problème. Pourtant je ne connais pas, et j'aimerais beaucoup me tromper, de recherche expérimentale de la xénophobie humaine fondée sur des hypothèses évolutionnistes. C'est ce qui fait le plus cruellement défaut à toutes les recherches connues : un point de vue ouvertement et constamment évolutionniste, c'est-à-dire fondé sur l'hypothèse que le racisme humain est de nature fondamentalement biologique, qu'il répond à un besoin puissamment ressenti de coopération sociale accrue et qu'il doit donc être examiné dans le cadre de la théorie de Charles Darwin. Si une telle recherche expérimentale était entreprise, alors de nouveaux faits apparaîtraient suggérant de nouveaux moyens d'intervention et les moyens habituels d'intervention, dont quelques-uns seront mentionnés à l'instant, prendraient une toute nouvelle dimension et un sens renouvelé.

\section{Les moyens habituels d'intervention}

La nature fondamentalement groupale du racisme - le fait qu'il est un produit de la vie en groupe - justifie la variété existante des approches utilisées tant sur le plan individuel que sur le plan social. Au niveau social, il est certain que le racisme doit continuer à faire l'objet de mesures légales et sociales propres à le combattre et adaptées à la configuration sociale locale. Aux États-Unis, par exemple, les mesures de programmes «d'action positive », le transport écolier visant à redistribuer les populations scolaires des minorités ethniques dans l'ensemble de la 
population scolaire totale semblent donner des résultats qui, bien que partiels, apparaissent indéniables; alors que dans d'autres pays ce sont d'autres mesures, comme la lutte contre l'antisémitisme ou visant à mettre fin à des privilèges d'origine religieuse d'une partie de la population qui seraient plus immédiatement utiles.

Mais il reste que ces interventions de type collectif et légal ne peuvent pas remplacer les efforts d'éducation et de rééducation qui sont requis pour produire une modification dans les comportements et pour les amener à favoriser chez tous les membres de la société un environnement de type égalitaire, remplaçant peu à peu le racisme ambiant par une attitude de compréhension et de soutien de la démocratie.

Cette dimension du problème raciste, la rééducation de la personne individuelle, pourrait utilement faire l'objet d'un renouvellement de la recherche. À cette fin, on peut rappeler le travail d'un pionnier américain de la lutte contre le racisme, Kurt Lewin ${ }^{1}$, vers la fin de la guerre et donc plusieurs années avant le début du mouvement américain des Civil Rights.

Lewin aborde le traitement du racisme par la même approche que celle qui est utilisée pour la modification du comportement dans une foule d'autres activités indésirables comme l'alcoolisme ou la délinquance : il y voit un processus de rééducation. Il soutient que les processus gouvernant l'acquisition du normal et de l'anormal sont fondamentalement les mêmes, que l'anormal en question concerne la criminalité, l'alcoolisme, le racisme, la prostitution, etc. Le processus rééducatif, dit-il, est essentiellement l'équivalent d'un changement de culture. Ce qui signifie pour notre propos :

1. Selon Lewin, la rééducation est un processus social dans lequel l'atmosphère particulière créée par le groupe est plus décisive ou importe davantage que l'acquisition de connaissances objectives exactes. Il prend exemple sur la rééducation menée chez les Alcooliques anonymes (AA), où l'atmosphère chaleureuse est plus efficace, pour aider le buveur à renoncer à l'alcool, que ne l'est le long et pénible apprentissage effectué dans le cadre d'un traitement médical. Il cite une étude décrivant le cas d'une contremaîtresse convaincue que la performance des ouvriers âgés est constamment médiocre, bien que tous les travailleurs âgés qu'elle connaissait personnellement dans sa propre zone de travail fussent au contraire assidus au travail et efficaces : ses préjugés étaient en opposition totale avec son expérience personnelle. Il en va ainsi du racisme, dit Lewin, qui ne pose pas d'abord et avant tout un problème de nature cognitive et l'on peut fort bien être raciste alors que la connaissance directe et l'expérience personnelle acquise lors de rencontres avec des personnes de l'autre groupe n'ont laissé uniquement que de bons souvenirs; des idées

Kurt Lewin, « Conduite, connaissance et acceptation de nouvelles valeurs ». 
objectivement exactes et même une pratique personnelle favorable ne sont pas suffisantes pour annuler l'effet des préjugés raciaux que l'on peut avoir.

2. De plus, la rééducation n'est pas davantage liée directement à une question de « volonté »: on peut continuer à être raciste tout en désapprouvant ce préjugé et même en faisant des efforts appréciables pour cesser de l'être.

Tout comme l'alcoolique sait qu'il ne devrait pas boire - et qu'il ne veut pas boire; ainsi le soldat américain de race blanche qui voit un Noir prendre rendez-vous avec une jeune fille blanche en Angleterre peut considérer qu'il ne devrait pas s'en formaliser - et il peut désapprouver consciemment ses préjugés. Néanmoins ses perceptions et sa réaction émotionnelle demeureront contraires à ce qu'il sait qu'elles devraient être ${ }^{1}$.

Il semble que ce qui importe avant tout et qui est décisif, dans le comportement d'une personne, soit le sentiment dominant dans le groupe auquel elle appartient.

3. Les stéréotypes incorrects ou les préjugés, dit Lewin, sont l'équivalent fonctionnel de faux concepts ou de théories fausses. Ce qui est requis c'est plus qu'un simple changement dans les idées, c'est un changement dans les perceptions et seul le groupe est en mesure de produire ce genre de résultat. Une illusion d'optique ne disparaît pas du seul fait que l'on sait qu'elle est fausse, on ne peut que tenter de la corriger en la replaçant dans son contexte adéquat au moyen d'expérimentations de type physique qui sont fréquemment très complexes et hors de portée de quiconque ne consent pas à faire le travail ardu que leur acquisition exige. Il en va de même du préjugé raciste, dit Lewin, qui est l'équivalent d'une théorie fausse. Les humains ont mis des siècles à comprendre pourquoi un bateau flotte, pourquoi un roseau émergeant de l'eau apparaît brisé, pourquoi le Soleil semble tourner autour de la Terre. L'acquisition de la bonne théorie suppose non seulement un changement dans les idées mais aussi dans la gestalt, dirions-nous, de l'ensemble des perceptions impliquées.

4. Enfin, l'acceptation d'un nouvel ensemble de valeurs et de croyances ne peut habituellement pas être suscitée à la pièce, c'est au contraire un processus global qui est en jeu et l'individu n'accepte le nouveau système de valeurs qu'à travers son appartenance au groupe. Ainsi, dit Lewin, la rééducation n'influence la conduite que lorsque le nouveau système de valeurs construit par le groupe en vient à dominer la perception de l'individu.

\footnotetext{
Op. cit., p. 7.
} 


\section{Empirisme}

On ne peut manquer d'être impressionné par le travail d'avant-garde accompli par Kurt Lewin dans l'immédiat après-guerre - il y a un demi-siècle déjà - pour développer une théorie cohérente et efficace de la rééducation et de la lutte contre le racisme. On appréciera tout particulièrement le fait qu'il ait pleinement reconnu l'importance du facteur psychologique et de la perception dans l'apprentissage et la diffusion des croyances et des préjugés, tout en refusant de ne voir dans le racisme qu'un effet psychologique et en insistant pour montrer à quel point, dans ce cas, le psychologique est asservi, en fait, à l'influence du groupe: au social. C'est l'influence du groupe qui est décisive, et non pas celle de la cognition de la psyché individuelle opérant de façon autonome.

Et pourtant, malgré toutes ces qualités qui font de Kurt Lewin un maître et qui confèrent à son intervention les caractéristiques d'une contribution exceptionnellement enrichissante à la question du racisme, il reste que son approche demeure en bonne partie empirique et que lui fait encore défaut le concept du principal facteur qui est à l'œuvre non seulement dans le racisme mais dans toute l'entreprise de rééducation elle-même en tant qu'elle est conditionnée par le groupe. Comment expliquer, en effet, l'existence de ce rôle si décisif du groupe ? Ce n'est pas tout de savoir que le rôle du groupe est décisif dans la rééducation, même si cette connaissance est fort utile en permettant au chercheur de franchir d'un seul coup le fossé qui a toujours séparé - et opposé l'une à l'autre l'explication invoquant exclusivement des facteurs psychologiques et l'explication invoquant exclusivement des facteurs sociologiques; encore faut-il savoir pourquoi ou comment le groupe en vient à acquérir cette importance décisive. Et ici, chez Lewin comme en général chez les psychologues et les sociologues de toute tendance, ce qui fait défaut c'est l'idée que le groupe est une création de la vie, qu'il résulte de forces présentes dans l'évolution. Ce qui manque, c'est le rattachement du groupe et de son rôle dans le cadre conceptuel général fourni par l'évolutionnisme de Darwin.

Comment en effet ne pas voir que, puisque seul le groupe est en mesure de corriger un comportement raciste, ou à tout le moins puisqu'on reconnaît en lui le facteur primordial capable de produire un tel résultat, on peut logiquement inférer que seul un groupe, assurément un autre groupe, a dû opérer en sens inverse dans l'apprentissage du comportement raciste ? Mais c'est justement là le genre de question que nous sommes ordinairement incapables de poser, y compris Kurt Lewin qui ne la pose pas. En fait c'est la question qu'on nous interdit d'habitude de poser en l'absence d'un cadre conceptuel adéquat autorisant la formulation de la question elle-même; un cadre qui ne serait pas susceptible de prêter flanc à l'accusation d'être lui-même raciste. La difficulté est donc de trouver une réponse non raciste à la question : pourquoi sommes-nous racistes ? 
Le problème posé, ici, est entièrement épistémologique et pour être en mesure de formuler cette question dans le cadre du discours cohérent il faut commencer par s'en donner les moyens. Or, seule la théorie évolutionniste nous permet d'entrevoir la totalité de ce qui est en jeu dans ce problème, seule la science de l'évolution autorise l'espoir d'échapper sur ce point précis à la tyrannie de l'ethnocentrisme.

On peut également voir à quel point l'existence du groupe rend parfois trompeuse ou malavisée l'opposition traditionnellement postulée entre l'« inné » et l'« acquis » en biologie. Lewin dit lui-même dans le texte cité qui date de 1945 que les spécialistes des sciences sociales sont d'accord pour dire que les différences qui existent aujourd'hui entre les hommes, Blancs, Noirs ou autres ne sont pas innées, et donc qu'elles sont acquises. Pourtant on pourrait soutenir qu'elles ne sont ni l'une ni l'autre car l'opposition qu'on veut voir entre l'« inné » et l'« acquis » n'est que partielle ou paradoxale et les conclusions qu'on peut en tirer peu probantes. L'origine du racisme ne peut certainement pas, pour le moment du moins, être attribuée à la génétique, en l'absence d'une preuve expérimentale en la matière ; il nous faudrait donc, selon le raisonnement traditionnel, reconnaître qu'il n'est pas « inné », mais ayant dit cela on ne peut pas davantage dire qu'il est « acquis » si ce que l'on entend par là signifie que dans certaines circonstances un être humain pourrait échapper à tout racisme. Le racisme est une fonction du groupe, et comme nous accordons généralement très peu d'importance au facteur groupe dans ce que nous pensons savoir de la constitution de l'être humain, il s'ensuit que les experts psychologisants et les sociologisants pourront sans doute longtemps encore se renvoyer leurs arguments respectifs sans jamais parvenir à s'entendre ou résoudre la question de manière satisfaisante.

Toutes ces difficultés sont levées lorsqu'on a recours à des concepts adéquats : le racisme est la forme humaine de la xénophobie animale, c'est le mot sale qui désigne l'ethnocentrisme du groupe, le système de croyances et de valeurs par lequel le groupe domine la perception de l'individu.

\section{Limites à l'usage du concept de xénophobie dans le cas des êtres humains}

Un dernier commentaire sur le racisme comme forme spécifiquement humaine de la xénophobie animale. Le flou qui accompagne inévitablement le concept de race se propage au concept de xénophobie et rend difficile sinon impossible l'assignation de limites nettes au domaine de la xénophobie humaine. Ce genre de problème ne semble pas apparaître dans notre connaissance des autres animaux. Dans tout groupe d'organismes non humains il est possible d'établir par voie expérimentale que, ou bien l'organisme est accepté par les autres membres du groupe, ou bien il ne l'est pas; ou bien il est porteur de l'odeur du groupe ou d'autres marqueurs usuels ou bien non ; ou bien sa mère est membre du groupe et le protège activement ou bien non. Chez les humains on peut s'attendre à ce que 
des facteurs de ce genre jouent un rôle analogue mais deux sortes de limites d'origine différente viennent y réduire la validité de ce genre de généralisation.

En premier lieu, dans le monde animal il ne semble pas y avoir de limites au rôle théoriquement pacificateur de la xénophobie. Chez les humains il pourrait en être autrement. L'être humain est un être capable de révolte et de comportements en apparence insensés qui, en dernière analyse, se révèlent bénéfiques au groupe. L'histoire humaine ne manque pas d'exemples d'entreprises politiques qui semblaient n'avoir que peu ou aucun avenir au départ et qui ont pourtant été menées en faisant basculer brusquement les paradigmes philosophiques ou culturels et les régimes politiques qui y prenaient appui. Il apparaît donc prudent de supposer qu'il existe de telles limites au rôle pacificateur de la xénophobie chez les humains. Seules des recherches expérimentales pourraient apporter réponse à cette question.

En second lieu et contrairement a ce qui se passe dans le monde animal, un groupe humain dispose d'un grand nombre de façons de rendre un membre étranger à son propre groupe, en lui retirant ou en réduisant la portée du titre de membre qu'il possède déjà, ce que les sociétés animales ne semblent pas en mesure de faire.

On pense d'abord aux conflits raciaux, évidemment. Au Canada les citoyens d'origine autochtone se sont longtemps et de multiples façons vu refuser certains des droits humains les plus fondamentaux. Le Parlement canadien a longtemps pris des mesures discriminatoires à l'encontre de certains autres citoyens, surtout d'origine asiatique. Au Québec certaines lois linguistiques récentes ont été injustes.

La xénophobie humaine apparaît aussi manifeste dans de nombreuses vexations qui peuvent être infligées aux minorités visibles ou à quiconque présente des traits que le groupe estime difformes ou inusités ; ce genre d'affront prend à l'occasion des formes particulièrement criantes dans les groupes d'enfants. Au cours des siècles passés l'exil, tel celui qui fut proposé à Socrate, a servi de moyen de contrôle social, possiblement comme moyen d'éviter l'application jugée inopportune de la peine de mort dans le cas de personnes appartenant à la bonne société. La procédure d'excommunication de la religion catholique a longtemps été en mesure d'exclure un individu ou une organisation, parfois une ville entière, non seulement de la vie religieuse mais aussi de la vie sociale elle-même, avec des conséquences potentiellement aussi meurtrières que les mesures similaires prescrites par la loi juive ou par la fatwa islamique. Les " chasses aux sorcières » qui ont eu cours pendant quelques siècles en Europe et moins longtemps en Amérique du Nord, la Terreur durant la Révolution française, les nombreuses purges menées par Joseph Staline au cours des années 1930 et jusqu'à sa mort, l'Holocauste mené par Hitler qui semble avoir été l'une des plus efficaces opérations de «nettoyage ethnique » de l'histoire de l'humanité, l'antisémitisme étant lui-même une des formes les plus tenaces de la xénophobie humaine en 
Occident ; sur un mode moins meurtrier, au cours des années 1950, la croisade anticommuniste du sénateur américain Joseph McCarthy contre les auteurs d'activités « anti-américaines »: toutes ces entreprises religieuses et politiques d'autojustification vertueuse furent inspirées au moins en partie par la xénophobie humaine et dirigées contre des membres appartenant déjà de plein droit à des communautés humaines.

Ce n'est pas tout. À notre époque de nombreuses mises en accusation et condamnations peuvent comporter la perte de droits civiques comme un couvrefeu personnel, la perte du droit de fréquenter certaines personnes ou endroits, la perte de l'usage d'un passeport ou d'un permis de conduire, toutes mesures ayant pour effet de réduire l'autonomie personnelle et le statut social de la personne à un état qui n'est pas celui d'un citoyen à part entière.

Un problème épistémologique surgit alors du fait que cette liste pourrait être prolongée presque indéfiniment sans jamais rencontrer de limites assignables, jusqu'à un point de généralité telle que le concept même de xénophobie perd toute signification et devient en pratique inutilisable : dira-t-on que toutes les guerres sont porteuses de xénophobie ? Qu'en est-il des luttes de castes, de classes et de religions? Est-ce que l'emprisonnement pour quelque motif que ce soit est xénophobique ? Le sexisme en est-il lui aussi ? De nombreuses questions de ce genre demeurent sans réponse, et bien qu'en certains cas il soit possible de leur apporter une réponse plus ou moins satisfaisante selon les convictions du locuteur et la nature de l'enjeu, il est clair qu'une telle extension indéfinie du concept de xénophobie humaine le rendra tôt ou tard aussi flou et sujet à caution que celui de racisme.

En résumé, chez les animaux non humains le concept de xénophobie est net, amplement documenté et utile, malgré l'absence de démonstration qu'il résulte effectivement d'une sélection naturelle. Appliqué à la conduite humaine son extension ne montre pas de limites indiscutables et il serait par conséquent souhaitable que son usage s'accompagne des précautions méthodologiques que l'on prend habituellement dans de nombreux autres cas impliquant l'application de concepts biologiques dans des contextes humains. Le concept de xénophobie humaine est fondamentalement biologique et peut être utile à la condition que ces limitations soient respectées. Ces limitations ne sont probablement qu'un indice de plus qu'il n'y a de sociobiologie qu'animale ; il n'existe pas véritablement, à l'heure actuelle, de sociobiologie spécifiquement humaine qui mérite le nom de science, tout comme, d'ailleurs, il n'y a pas de science biologique particulière aux seuls humains. La xénophobie humaine ne fait pas exception.

\section{Quelques autres questions morales. Le sexisme.}

La question du racisme a été évoquée plusieurs fois dans cet ouvrage parce que c'est l'un des plus pressants de tous les problèmes affectant notre conscience 
morale actuelle de façon plus ou moins permanente. C'est certainement le domaine où une recherche expérimentale effectuée par une science de la morale serait la plus souhaitable et prometteuse.

Mais on ne voit pas clairement de limites au domaine humain qu'une recherche expérimentale guidée par un point de vue évolutionniste peut explorer et éclairer. Un grand nombre de pratiques humaines courantes pourraient bénéficier d'une approche expérimentale cherchant à identifier leurs bases biologiques et les résultats moralement indésirables qu'elles produisent à l'occasion. Parmi elles on trouve le sexisme. Il y a quelques raisons, non concluantes par elles-mêmes mais néanmoins réelles, de croire à l'existence d'une base biologique du sexisme humain ${ }^{1}$. Les rares connaissances objectives dont nous disposons et qui sont théoriquement utilisables dans le dossier du sexisme nous persuadent que si l'on voulait vraiment mettre fin à l'infériorisation sociale de la femme il faudrait mettre en œuvre des moyens beaucoup plus énergiques, plus systématiques et à plus long terme que tout ce qui à ma connaissance a été proposé jusqu'ici sauf peut-être par les groupes féministes les plus radicaux et les moins écoutés.

Ce n'est la qu'un cas, somme toute banal parce qu'on le retrouve dans la plupart des religions pratiquées en Occident. À l'heure actuelle il existe des féministes chrétiennes, des féministes musulmanes et des féministes juives qui protestent, chaque intervenante demeurant à l'intérieur de sa propre religion mais chacune disant essentiellement la même chose, à savoir que les textes de leurs trois Révélations respectives n'ont jamais autorisé expressément le redoutable sexisme des mâles qui s'est emparé de la fonction administrative et politique de leurs Églises et en ont fait leur chose en détournant les objectifs explicites des textes à des fins qui sont les leurs en tant que mâles. De la sorte, pendant que les textes dits sacrés demeurent (presque par définition) inaltérés à travers les siècles, les interprétations politiques sexistes de ceux qui s'en réclament leur font subir une « dérive » qui demeure incompréhensible et qui désole quiconque n'a accès qu'au libellé du texte. Ici encore une approche évolutionniste semble toute indiquée, indispensable en fait, à qui veut comprendre la nature des processus susceptibles de produire de tels résultats.

Nous avons, plus haut, abondamment fait état des limitations inhérentes à l'efficacité réelle de toute contrainte secondaire, législative ou simplement éthique, qui tente avec des moyens rudimentaires ou empiriques de remédier aux effets jugés indésirables ou pervers d'une contrainte primaire mal identifiée ou dont l'existence est ignorée purement et simplement. Une recherche expérimentale sur le sexisme qui serait basée sur la théorie évolutionniste apparaît en revanche fort prometteuse de connaissances nouvelles et utiles d'un point de vue humaniste.

Une partie de ce dossier fort complexe est résumée au chapitre 4 de Lagadec (1982), Dominances : « La dominance sexuelle ». 


\section{La jalousie}

Il serait également souhaitable d'étudier par voie expérimentale la jalousie humaine, définie comme le sentiment de propriété exclusive qu'une personne peut éprouver à l'égard des activités amoureuses de son ou ses partenaires sexuels ; on sait que la jalousie est l'un des vecteurs les plus fréquents de l'homicide. On peut également songer à la rancune ou au dépit, pour des raisons analogues. À l'envie, définie comme le sentiment de chagrin haineux que nous inspirent le bonheur ou les avantages d'autrui. À l'agressivité et à la violence, évidemment. Au remords et à la culpabilité facilitant la socialisation et le conformisme. C'est tout un Traité des passions qu'il serait possible de réécrire de ce point de vue.

Il en va de même de l'usage apparemment aussi ancien que l'humanité de tous les alcools et produits euphorisants, excitants, narcotiques, sédatifs, somnifères, stupéfiants ou tranquillisants qui servent parfois à des fins religieuses ou de productivité industrielle et sont aussi d'importants vecteurs de la criminalité : pourquoi le groupe humain semble-t-il pratiquement incapable de se passer de ces " paradis artificiels » même lorsque leurs conséquences néfastes sont si évidentes en termes de physiologie, de psychologie, de criminalité, d'économie et de vie familiale et sociale ? Il y a lieu de chercher à savoir s'il n'y aurait pas, dans la biologie humaine, une base empiriquement identifiable à l'origine de cette constante de notre existence.

\section{De l'immoralité de la théorie de l'égalité}

Encore un peu de théorie. L'idée principale de l'approche adoptée dans cet ouvrage est que la morale humaine opère à deux niveaux différents, primaire et secondaire, et que cette dualité peut entraîner des résultats opposés ou antagonistes. Exemple, l'immense problème de l'avortement opposant la contrainte primaire du soin dû à notre progéniture à la contrainte secondaire du droit de la femme à la libre disposition de son corps et qui relève des droits de la personne. Dans l'état actuel des mœurs occidentales on peut prédire qu'un grand nombre des plus forts opposants à la pratique de l'avortement auront amplement le temps de mourir avant de commencer à comprendre ce qui leur arrive et l'enjeu du problème. Où est l'égalité dans tout cela?

Revenons, pour le voir, au racisme. L'Amérique du Nord accorde une grande importance à la science et à l'idée d'égalité mais elle continue néanmoins de donner des preuves évidentes de son racisme. On trouve, d'abord au niveau de la contrainte primaire de la xénophobie animale, l'évidente persistance du racisme, alors qu'au niveau de la contrainte secondaire de la morale dite ou éthique, dans une inconséquence flagrante, nous sommes fiers de prétendre que tous les humains sont égaux. La réalisation de cette dernière affirmation continue, lentement, à gagner du terrain d'une année à l'autre en dépit de la remarquable persistance de la

morale raciste. 
Je crois que nous avons là un exemple parfait de production et de croissance incoercibles de la « mauvaise herbe » de l'immoralité, car en dépit de la très large diffusion faite à la théorie morale et politique de l'égalité et en dépit du soutien que celle-ci peut apporter à la lutte contre le racisme, il reste que cette théorie rate complètement la cible et ne commence même pas à s'attaquer au vrai problème qui est celui de la xénophobie animale. Avec le résultat que plus nous réalisons et affirmons que nous sommes de plus en plus égaux grâce à la démocratie et à la pratique de la science, ce qui est fréquemment vérifiable, plus nous devenons conscients de l'effroyable immoralité impliquée par les persistantes inégalités raciales devant lesquelles nous nous sentons impuissants et qui sont alors imputées à la mauvaiseté individuelle et non pas à leur base réelle qui est celle de la xénophobie animale. Le problème, quand on le formule sur le plan théorique, est que nous essayons de guérir le symptôme et ne réussissons qu'à alimenter notre sens particulier d'immoralité, bien au chaud que nous sommes dans le confort et l'ethnocentrisme de notre groupe qui nous persuade de l'excellence de notre théorie égalitariste lors même qu'elle s'accompagne de si piètres résultats. Tout se passe comme si cette inconséquence vérifiable était par elle-même sociogène et que la solidarité sociale se fortifiait chaque fois que notre groupe trouve moyen de conforter son consensus sur la bonne manière, la nôtre, d'appliquer le double standard de moralité tout en procurant à ses membres le luxe de continuer à pontifier sur la morale égalitaire sans avoir à renoncer aux avantages hiérarchiques dévolus à tous les groupes de primates.

Après tout, pourquoi croyons-nous que nous sommes égaux ? La question peut sembler sotte ou insolente mais la réponse est de grande conséquence. En termes plus explicites, pourquoi le sens moral occidental a-t-il perdu la bienheureuse certitude «bénie par Dieu » qu'avaient nos arrière-grands-pères, qui n'étaient pas sots, de l'incontestable supériorité de notre race et de la forme bien particulière de notre ethnocentrisme, au seul profit apparent de cette déchéance psychologique et sociale de notre actuelle culpabilité sur le problème du racisme ? En termes plus politiques et plus polémiques, pourquoi la bonne foi, la générosité chrétienne et le rationalisme kantien qui ont présidé à l'édification de l'empire colonial anglais et de l'empire colonial français (et de leurs avortons belge, allemand et italien) sontils dorénavant déclassés, pourquoi leur simple évocation est-elle maintenant devenue insupportable ? Le problème posé concerne le changement intervenu au cours de ce siècle et de celui qui l'a précédé dans la morale occidentale : pourquoi la morale change-t-elle?

La question est redoutable, assurément, et ses conséquences immenses car si nous parvenions à savoir comment la morale en vient parfois à changer nous commencerions à comprendre pourquoi il y a de l'histoire. Essayons tout de même.

Beaucoup de choses, assurément, concourent à influencer la configuration du sentiment moral et les diverses formes qu'il adopte à travers les âges. L'une de ces influences est exercée par la perception que nous avons des choses, la gestalt 
propre à chaque époque dans chaque culture, et cette perception est à son tour modifiée par l'action que nous exerçons sur les choses. En ce sens je crois que le moteur du changement dans le sentiment moral pourrait être sans rapport ou fort éloigné de la question raciste ou de tout effort conscient et délibéré que l'on pourrait faire pour ne pas être soi-même réputé raciste. Je crois, à la suite de Marshall McLuhan, que la pensée morale tend à épouser la forme de pensée qui est à l'œuvre dans la production des concepts, des instruments et des produits techniques qui constituent l'essentiel de notre vie physique et sociale et qui reviennent nous hanter par après, comme dans La Tentation de saint Antoine de Jérome Bosch, au fur et à mesure qu'ils prennent forme matérielle et deviennent les miroirs inversants (dans le miroir la main gauche est une main droite) des passions qu'il nous plaît d'appeler nos identités et nos âmes. Dans notre société ce moteur est principalement la science et sa créature la technique. Notre formation scientifique et technique peuple notre univers de concepts et de choses dotés du statut très peu naturel de purs « objets » tel qu'expliqué plus haut, cette pureté étant la fiction favorite de la science positiviste occidentale selon laquelle toutes les choses du monde sont égales en ce qu'elles sont toutes moralement vierges, également dépourvues de qualité morale intrinsèque. Ce laminoir universel qui atteint maintenant les derniers refuges de notre espérance est inexorablement exigé par la transformation de toute chose en « objet » qui la rendra susceptible d'un traitement scientifique. Quand la némésis revient nous hanter par la suite il est trop tard, nous sommes déjà narcosés (narcose veut dire sommeil, demi-dieu Narcisse et quart-dieu cocaïne) par la découverte que notre sens moral historiquement légué par la longue tradition juive-grecque-chrétienne est déjà obsolété cependant que, par le biais des sciences sociales, l'ensemble de l'entreprise scientifique a déjà commencé à faire de chacun de nous un " objet » vidé de toute vertu intrinsèque. Dorénavant privés du savoir naïf indiscutable qu'avaient les Anciens de ce que c'est que d'être un homme, nous acquérons progressivement la mine hagarde qui convient aux immigrants livrés en pâture à ce nouvel univers construit par la science. Nous possédons dorénavant toutes les qualités qui feront de nos précieuses personnes d'excellents «objets». Ce résultat était probablement inévitable. Nous croyons que nous sommes égaux parce que nous croyons a la science. 


\section{Chapitre 5}

\section{L'improbable}

$\underline{\text { Retour à la table des matières }}$

Il reste à indiquer quels objectifs il conviendrait de donner à une morale humaine qui disposerait d'une science morale. Cette question des objectifs doit être posée dans un contexte aussi large que possible, je veux dire dans un contexte non seulement de science mais aussi de philosophie et de culture.

\section{L'affaiblissement de la morale spontanée}

Le présent essai de formulation d'une théorie morale n'est qu'une contribution après tant d'autres dans le cadre d'un développement qui, depuis plus d'un siècle, dépossède peu à peu mais d'une façon apparemment irrésistible la morale spontanée d'un grand nombre de ses anciennes attributions et de ce fait d'une bonne partie de l'autorité autrefois attachée à ses énoncés.

J'appelle morale spontanée le jugement moral basé uniquement sur l'ethnocentrisme qui nous est propre et qui ne tient aucun compte de l'existence de faits objectifs susceptibles d'occasionner à eux seuls les conduites humaines mises en cause par notre jugement, ni pour la science éventuellement capable d'apporter son aide à l'identification de tels faits. L'expression « morale spontanée » veut dire deux choses : 1) c'est une morale pré- ou antiscientifique ; 2) la morale spontanée, par définition, est la morale du groupe avant d'être celle de l'individu auteur d'un énoncé particulier.

On sait et nous avons dit comment, depuis plus d'un siècle, l'anthropologie, la criminologie, la sociologie, la médecine sociale, la psychologie sociale et bien d'autres sciences encore ont transformé d'anciennes questions morales en questions plus objectives, en questions apparemment « dé-moralisées » pour ainsi dire, objectivées, et ont ainsi aidé à identifier une série de conditions objectives reconnues pour donner naissance à des comportements humains jusque-là uniquement considérés comme immoraux. Grâce à la contribution de ces sciences 
ces comportements deviennent alors plus objectivement compréhensibles. De nos jours cet affaiblissement de la morale spontanée semble engagé dans une voie irréversible et à première vue sans limite.

Au siècle dernier, par exemple, le suicide était souvent jugé immoral et le législateur pouvait le réprimer par voie légale. En Angleterre le suicide (ou la tentative) a longtemps été un délit et ce n'est qu'en 1961 qu'il a cessé d'être réprimé et puni par la loi. Pourtant dès 1897, le sociologue Émile Durkheim ${ }^{1}$ montrait que plusieurs conditions objectives protègent relativement l'individu du suicide, alors que d'autres créent des groupes a risques. Selon son étude restée célèbre et qui fut souvent confirmée par de nouvelles études par la suite, le simple fait d'être marié protège une personne du suicide et cette immunité relative est plus grande encore quand les époux ont des enfants; il en va de même du rôle de la religion catholique et davantage encore de la religion juive qui protègent mieux leurs fidèles respectifs du suicide que ne le fait la religion protestante. Il en va de même encore de certaines occupations comme l'agriculture qui protègent davantage du suicide que ne le font les professions libérales et commerciales.

Ce genre d'études, qu'on rattache de nos jours à l'épidémiologie qui est au croisement de la médecine et des sciences sociales, a pour effet de transformer une ancienne question morale en une question de type plus objectif, rendant ainsi possible une intervention ponctuelle ou à long terme susceptible de modifier la situation que l'on se contentait jusque-là de condamner comme immorale. Dorénavant, donc, le suicide relève davantage de la sociologie et de la médecine, et moins de la morale spontanée et des tribunaux. Cette contribution de la science sociale réduit généralement les faits moraux à n'être plus que des faits sociaux, et ces faits sociaux seront à leur tour considérés comme relativement indépendants de la conscience morale individuelle.

C'est dans ces conditions que l'on assiste, depuis un siècle, à un recul partiel mais régulier et croissant de l'importance et de l'ascendant social autrefois associés à la morale spontanée. C'est de ce développement dont se sont prévalus nombre de scientifiques, en particulier les tenants du positivisme, pour croire que, de même qu'au cours des derniers siècles la physique et l'astronomie ont éliminé la théologie et l'astrologie du discours cohérent moderne, de même ce n'est plus qu'une question de temps avant que le discours moral tout entier disparaisse du discours cohérent occidental. Pour certains scientifiques ce n'est pas une simple question d'opportunisme mais plutôt d'hygiène mentale et de logique politique.

Je pense avoir montré que cette dernière opinion repose sur une illusion. Il y a des limites à l'affaiblissement indéfini de la morale spontanée. Ces limites sont imposées par les groupes particuliers à l'intérieur desquels nous vivons et

Émile Durkheim, Le Suicide. Étude de sociologie. [Texte disponible dans Les Classiques des sciences sociales. JMT.] 
travaillons et qui fournissent les données de base de notre sens moral. L'ethnocentrisme provient du groupe et demeure à son service. J'ai présenté les raisons nous portant à penser que l'ethnocentrisme humain semble actuellement indépassable ; dans un grand nombre de questions où ses intérêts sont impliqués, l'ethnocentrisme parvient maintenant à mettre la science à son propre service, et nous avons toutes les raisons de penser qu'il continuera de le faire dans l'avenir. Il est certain que la science veut être value-free, sans connotations morales mais la science n'est pas elle-même un objet de science, sinon d'une de ces sciences sociales qui n'est pas davantage value-free et elle demeure donc, tout au moins en théorie, livrée à un ethnocentrisme qui n'est qu'un ethnocentrisme parmi d'autres, parfois plus puissant que d'autres, un instrument de pouvoir dans le jeu des rapports existant entre les diverses ethnies et les divers groupes humains. Savoir n'est pas toujours pouvoir mais c'est toujours une force dans un rapport de forces.

De plus j'ai aussi montré que, tout comme la science médicale n'a pas éliminé la maladie mais seulement fourni un moyen efficace de l'identifier et d'en combattre les effets indésirables, la biologie évolutionniste, base commune à une morale scientifique et à la médecine, n'éliminera pas la morale ni par conséquent l'immoralité qui en est un sous-ensemble. Comme il a été dit plus haut, une société humaine sans immoralité est une impossibilité théorique. La biologie évolutionniste peut donc seulement nous fournir des moyens efficaces d'identifier l'origine des différentes formes locales d'immoralité et possiblement d'en réduire certains effets. Quels effets ? Ceux que nous jugerons indésirables. Et au nom de quoi les jugerons-nous indésirables? Au nom d'une morale ethnocentriste, évidemment.

Sur l'ensemble de cette question concernant la morale et le sort que lui fera la science de l'avenir, l'origine de l'erreur du positivisme scientifique est la confusion qu'il continue d'entretenir encore très vivante entre ce qu'est la science et ce qu'est la nature. Le positivisme, qui est une philosophie et non pas une science, est l'ethnocentrisme particulier au monde scientifique actuel, c'est là sa marque de commerce, il faudrait l'appeler le scientisme.

Cette affirmation, à l'effet que le scientisme est la forme particulière d'ethnocentrisme de la communauté scientifique appelée positiviste, ne résulte pas d'un changement subreptice de définition du concept d'ethnocentrisme ni d'une autre manœuvre conceptuelle. Le moteur principal de l'ethnocentrisme n'a pas été attribue a un groupe ethnique particulier mais à l'appartenance à un groupe quelconque, à tout groupe tant d'être appelé une tribu dans la terminologie de Hardin et appliquant le double standard de moralité.

Le scientisme est bête car l'ethnocentrisme sous toutes ses formes est toujours bête et il pue. Lorsqu'il prétend abolir toute morale le scientisme a recours au plus vieux truc du monde qui consiste à asseoir le monopole de sa propre morale et de son propre système d'obligations et d'interdits sur l'interdiction de toutes les 
(autres) morales. Dans l'histoire de l'ethnocentrisme c'est là un procédé normal ou fréquent, mais dans ses formes les plus sublimes le scientisme peut se hisser jusqu'aux sommets atteints en 1933 par Alfred Korzybski ${ }^{1}$ qui a soutenu dans son livre Science and sanity que la science c'est la santé. La thèse veut être entendue au sens littéral et associe continuellement le discours non scientifique à l'aliénation mentale pure et simple.

Croire que la science est la même chose que la nature c'est commettre la faute logique la plus grossière et prendre la partie pour le tout, c'est confondre la carte et le territoire.

Cela dit je n'ai pas du tout l'impression que le présent ouvrage contribuera à faire reculer la science, comme le veut une longue tradition de la philosophie morale, je pense au contraire qu'il contribuera à la faire progresser car ce genre de débat appartient au travail quotidien par lequel la science se développe historiquement et se construit. Pourtant, et malgré ce qui vient d'être dit, il faut quand même rappeler que le présent ouvrage n'est pas un ouvrage de science mais de philosophie et c'est pourquoi il devient nécessaire de poser la question des objectifs dans un cadre non limité à la science mais dans celui, plus vaste, de la philosophie et de la culture en général. Ce qui veut dire, en pratique, dans le cadre d'un humanisme.

Qu'adviendra-t-il et quel objectif conviendrait-il d'assigner a une morale humaine qui, dans son coffre à outils, disposerait d'une science morale ? À une morale qui ne serait donc pas tout à fait spontanée, peut-être d'ailleurs à aucune époque la morale n'a-t-elle jamais été tout à fait spontanée ni assurément tout à fait scientifique, mais un peu les deux à la fois.

\section{Nietzsche et la philosophie}

Ma réponse à ces questions commencera par un rappel de ce que la présente entreprise doit au philosophe allemand Friedrich Nietzsche. C'est à lui que j'ai emprunté l'idée centrale du présent ouvrage à l'effet que les valeurs sont des contraintes. Nietzsche a dit que la majeure partie des bonnes actions que nous posons et qui sont conformes au devoir n'ont aucune valeur éthique parce qu'elles résultent de la contrainte. Il voulait dire qu'elles nous sont imposées par le groupe. Sa Généalogie de la morale ${ }^{2}$ a montré que la plus grande partie de ce que nous appelons notre vie morale n'est pas le produit de la conscience individuelle, encore moins d'un choix, mais qu'elle est au contraire sociale et grégaire, rendue obligatoire par le troupeau. Je répète que la plupart des gens la plupart du temps

Alfred Korzybski, Science and Sanity. An Introduction to Non-Aristotelian Systems and General Semantics.

2 Friedrich Nietzsche, Euvres philosophiques complètes. 
pensent et agissent comme leurs voisins, lesquels, en général, ont été choisis précisément pour cette raison.

C'est la première partie de ce que j'ai voulu montrer dans ce livre. J'ai voulu montrer que notre morale consiste en ceci que nous sommes contraints, et ce de deux façons. La première est que nous sommes des animaux, des primates. Cela, Nietzsche, pour sa part, ne le savait pas ou ne voulait pas le savoir ; bien qu'il ait été au plus haut point préoccupé par l'existence du corps, il ne voulait pas le savoir dans le sens que nous appelons aujourd'hui scientifique et qui est celui de la biologie comme science de la vie. Il n'a rien compris à la théorie évolutionniste de Darwin qui était son contemporain. Ce qui n'avait rien d'exceptionnel à son époque ; Ernst Mayr, l'un des grands noms de la théorie évolutionniste aux ÉtatsUnis a remarqué :

Après-coup, on s'étonne de voir à quel point les philosophes de l'époque, au courant des idées scientifiques ont méconnu Darwin ou n'ont même pas tenté, soit d'incorporer ses concepts aux leurs, soit à tout le moins fait un effort sérieux pour le réfuter adéquatement ${ }^{1}$.

En deuxième lieu, nous sommes aussi contraints parce que nous vivons en groupe, et cela, cette fois, Nietzsche le savait si bien que pour lui la morale humaine coïncide pratiquement ou est une description sur le mode impératif de la vie sociale du troupeau. Objectivement il avait raison, mais pour nous le problème vient de ce qu'il avait encore plus raison qu'il n'a pu le croire lui-même, car les contraintes qu'il a si longuement et brillamment dénoncées de son propre point de vue moral, qui y voyait l'influence du troupeau, naissent en fait de l'évolution de la vie. Il n'a pas vu a quel point cette origine biologique confère à ces contraintes une influence sur le comportement humain qui est beaucoup plus grande et plus durable que tout ce qu'il a pu imaginer.

De plus, le problème se complique encore du fait qu'il n'y a pas chez Nietzsche de théorie du social et qu'il ne peut pas y en avoir, sauf évidemment ce qui en tient lieu dans la philosophie occidentale et qui est la fable du contrat social à la manière de Hobbes et Rousseau. Par théorie du social, j'entends une réponse articulée et raisonnable à la question suivante : pourquoi vivons-nous en société ? Par conséquent il faut convenir que le troupeau dont parle Nietzsche n'est pas une réalité mais seulement une métaphore puisqu'il ne lui donne pas un sens biologique, évolutionniste. Je dis qu'il ne pouvait pas y avoir chez lui de théorie du social au sens que la philosophie de Nietzsche est l'un des plus beaux fleurons de la pensée occidentale persuadée que la vocation de l'individu et de la culture humaines consiste à surmonter les forces présentes dans le troupeau, qui sont en fait les forces de la vie. C'est sur ce point en effet que se joue le rôle de la société

Ernst Mayr, One Long Argument. Charles Darwin and the Genesis of Modern Evolutionary Thought, p. 50. 
dans l'ensemble de la question morale: est-ce que la pensée humaine, en particulier la pensée morale, est plus puissante que la vie ou encore plus avisée ?

Ceci mérite d'être dit avec plus de netteté, et puisqu'il s'agit de donner la réplique à la fable du contrat social on conviendra de l'à-propos d'un recours au mythe. Dans le récit de la Genèse la pensée occidentale se persuade qu'au sixième jour le Dieu juif n'a guère besoin que de deux ingrédients pour créer Adam et Ève : d'abord un peu de terre ou de limon pour leur donner un corps, ensuite un " vent » pour leur insuffler une vie dans la bouche ou le nez. Et c'est à peu près tout, il n'avait aucun besoin d'ajouter une dimension sociale à ses créatures parce qu'en Occident judéo-chrétien la sociabilité n'est pas une partie constitutive de la nature humaine. Jean-Jacques Rousseau va jusqu'à dire dans le Contrat social : "Celui qui ose entreprendre d'instituer un peuple doit se sentir en état de changer pour ainsi dire la nature humaine ${ }^{1}$. » Il ne faudrait pas croire que cette croyance soit aujourd'hui dépassée, elle demeure au contraire très active et ses effets sont visibles, depuis les premières institutions de la Révolution française jusqu'à la Révolution bolchevique qui prétendait abolir l'État et les inégalités sociales.

C'est que pour tout Occidental la dimension sociale de la vie humaine est pour ainsi dire un accident de parcours, jetable, il se persuade qu'elle ne lui advient qu'après-coup, longtemps après que l'être humain a été constitué dans sa véritable essence. Dès que l'être humain devient social son sentiment est qu'il est perdu, son entrée dans le social lui apparaît comme une conséquence de la Chute, c'est là son malheur, croit-il, une déchéance qu'il n'a pas méritée et qui n'aurait jamais dû lui advenir.

Là-dessus Nietzsche ne différait guère des autres philosophes d'Occident. Il croyait en son temps, tout comme la plupart de ceux qui se réclament de lui continuent de le croire de nos jours, non seulement qu'il pouvait mais qu'il devait échapper au troupeau et à la morale du troupeau. Il fut l'un des premiers à comprendre que le Bien et le Mal sont l'invention du troupeau, et toute son œuvre s'évertue à dépasser cette règle que le troupeau impose. Dans son esprit la morale humaine ne méritera ce nom que lorsqu'elle aura résolument laissé derrière elle la règle du troupeau. Bien que Nietzsche ne soit évidemment pas rousseauiste et ne croie pas que l'homme naisse bon mais plutôt qu'il naît de la victoire d'Apollon sur Dionysos, il reste que, pour des raisons différentes, son opposition à l'État est aussi totale que celle de Rousseau. Ce qui n'est pas une théorie du social mais seulement un moyen parmi d'autres d'empêcher que la question de l'origine du social soit posée.

Si bien que sur l'ensemble de la question morale Nietzsche a pu adopter à l'occasion des positions qui paraissent étonnamment proches de celles des positivistes d'aujourd'hui (qui n'ont pas davantage de théorie du social). Car,

Euvres complètes, tome I, II, Ch. VII, p. 655. 
comme les positivistes de notre époque, il lui arrivait de rêver de ce qu'il appelait un idéal de liberté débarrassé de toute morale. Dans un fragment posthume datant de l'automne 1887, Nietzsche écrit : «On doit restreindre et circonscrire pas à pas le domaine de la morale. » Dans le même paragraphe il dit également :

... un degré de hauteur serait concevable où le concept de "vertu » serait éprouvé de telle sorte qu'il aurait l'accent de virtù, vertu de la Renaissance, vertu libre de toute morale. Mais pour l'instant - que nous sommes encore loin de cet idéal ${ }^{1}$ !

Sur ce sujet Nietzsche avait tort et sa tentative est un échec. Je pense avoir montré qu'une telle entreprise est nécessairement vouée à l'échec. Nous sommes des êtres moraux parce que nous sommes sociaux et parce que la vie de groupe impose à l'exercice de la liberté individuelle des contraintes que nous subissons sous une forme ou sous une autre. Le Bien et le Mal sont effectivement définis par le groupe comme Nietzsche l'avait vu, mais la même raison fait qu'il n'y a pas d'Au-delà du bien et du mal comme il a cherché à le montrer, il n'y a pas eu d'«inversion de toutes les valeurs", ni de son vivant ni par la suite, et pas davantage de Surhomme parce qu'il n'est pas du pouvoir de la personne, fût-ce celle du plus libre des hommes, d'abolir l'influence décisive du groupe et l'ethnocentrisme de sa pensée marsupiale.

On pourrait possiblement attribuer son échec à définir un Au-delà du bien et $d u$ mal à l'absence chez lui, encore que ce raisonnement n'est évidemment pas causal parce qu'on ne peut jamais dire qu'une absence soit une cause, d'une généalogie de la société qui aurait prolongé sa justement célèbre généalogie de la morale décrivant l'influence du troupeau. Cet échec en annonçait un autre tout aussi durable et qui devint avec le temps plus notable encore, celui des sciences sociales à se débarrasser une fois pour toutes de l'évaluation morale au profit d'une hégémonie totale du discours cohérent. Les plus que centenaires sciences sociales ne sont toujours pas value-free et ne peuvent donc pas, à strictement parler, prétendre être des sciences. Mais puisqu'elles persistent cependant à maintenir leur prétention à un tel statut scientifique, peut-être aurions-nous intérêt à porter davantage attention à la nature de leurs véritables rôle et fonction. En bref : dans la mesure ou ces prétendues «sciences sociales » n'en sont pas, que peuvent-elles être si ce n'est de la philosophie ? C'est Charles Darwin (et non pas les « sciences sociales » ni Marx qui en a longtemps rêvé) qui, en l'enchâssant dans la science de la vie, nous a fourni le concept d'histoire se rapprochant le plus de ce que l'on attend d'une science.

Hors de la considération de cet échec particulier de Nietzsche, le présent essai demeure nietzschéen d'esprit, attentif à ce que Nietzsche nous a enseigné et à l'immense influence de ses écrits sur la pensée morale d'Occident. C'est grâce à sa vision que nous pouvons mieux comprendre de nos jours, ce qui n'était sans doute

Euvres philosophiques complètes, tome XIII, p. 127. 
guère possible à son époque, que si la plupart de nos bonnes actions nous sont imposées par le groupe c'est qu'elles sont d'origine animale et que leur loi est celle de la biologie évolutionniste. C'est parce qu'elles relèvent de la biologie et non pas d'un choix conscient que nos contraintes, notamment notre altruisme, s'imposent à nous avec tant de force.

Pourtant, et en dépit de cette ignorance qui fut la sienne, peu de philosophes d'Occident auront été aussi conscients qu'il l'était du corps humain et de ses exigences. Et je trouve très cruel de songer que lui-même, pour sa part, aurait été infiniment heureux d'apprendre que l'espèce humaine est le produit d'une évolution opportuniste et sans but. On peut douter qu'un autre philosophe ait jamais existé à l'exception possiblement d'Héraclite - qui eût été aussi absolument ravi à l'idée que la vie n'invente de nouvelles formes que par une évolution elle-même opportuniste et sans but, à partir de mutations qui sont le fruit du hasard, d'écarts produits dans la transmission du matériel génétique d'une génération à la suivante et par brassage génétique aléatoire, tous événements d'une très haute improbabilité.

\section{L'improbable}

C'est manifestement cette très haute improbabilité qu'il faut assigner comme objectif, comme monde à explorer et à inventer, à une morale qui disposerait d'une science morale. Cette quête de l'improbable serait un équivalent scientifique et éthique du serment d'Hippocrate disant : essaie d'être utile mais assure-toi de ne pas nuire. La formule de la recherche de l'improbable au moyen d'une science morale pourrait être : mets le probable de la science au service de l'improbable de la vie, mets la science au service de l'art.

Je me hâte de préciser qu'il n'y a là nul éloge de l'anarchie. Il suffit de gratter un peu la surface de toute théorie anarchiste pour mettre à nu une vision disant quelque chose comme : débarrassons-nous tout d'abord de ces généraux, présidents et chefs de police, et dès ce moment les humains recouvreront spontanément l'harmonie de leur vraie nature : en dépit des apparences qui nous portent à penser le contraire l'anarchie demeure sourdement déterministe. La recherche de l'improbable est beaucoup plus exigeante. La liberté est l'essence de la vie et non pas une section réservée à l'usage exclusif des primates parlants dans le stade de l'univers. La liberté ressemble davantage à ce que serait une perpétuelle et mouvante réinterprétation de la loi de la gravitation qui aurait pour effet de favoriser la production de formes toujours nouvelles de lévitation.

La recherche de l'improbable rend en pratique l'entreprise artistique, qui s'attache à la production de ce qui est unique, indiscernable de la créativité morale et de sa quête de vérités d'un ordre plus grand et de plus hautes formes de vie. C'est peut-être la raison qui fait que, dans nos propres sociétés, la valeur de certains produits de l'art est profondément bien qu'obscurément reconnue alors même qu'ils violent manifestement les règles de conduite reconnues et les mores de l'époque. 
En d'autres mots la recherche morale de l'improbable est l'exact contraire de la méprisable moralisation. Nous méprisons le discours moralisateur parce qu'il attriste l'improbable jusqu'à le réduire à la banalité du probable exigée par le troupeau.

On voit donc ce que ce livre doit à Nietzsche. C'est lui qui nous a enseigné que la morale a une généalogie ; que l'établissement de cette généalogie peut nous faire découvrir le rôle qu'y joue le troupeau; c'est lui qui nous a persuadés que ce troupeau est bête et que le rôle de la philosophie est de le montrer. C'est ce que dit Gilles Deleuze commentant Nietzsche : le rôle de la philosophie est de rendre la bêtise honteuse ${ }^{1}$.

C'est contre la bêtise du groupe que la morale peut lutter. Pas contre le groupe réel ou historique, car cette lutte-là, d'autres groupes s'en chargent habituellement qui peuvent être tout aussi bêtes. Que le bon Dieu bénisse tous les groupes historiques, ils sont de la vie! Il faut plutôt lutter contre ce qui sollicite et encourage la force et la bêtise du groupe, contre ce qui en nous est tenté de donner des armes au policier du probable pensionnant dans notre tête.

Nul n'est à l'abri de la pensée engendrée par le troupeau. La pensée du troupeau se reconnaît à ceci qu'elle me prive de ma dignité. Ma dignité c'est l'honneur pour l'animal que je suis d'être l'homme que je suis. Alors que pour la pensée du troupeau le sens de l'honneur est plus directement lié à la biologie et au sexe, pour le troupeau le sens de l'honneur concerne uniquement les mâles (les femmes sont censées ne rien connaître à l'honneur) et à leur maîtrise de la sexualité de leurs femmes, toutes leurs femmes : mères, soeurs, épouses et filles. Dans tous les pays où les mâles ont perdu cette autorité sur la sexualité de leurs femmes leur sens traditionnel de l'honneur s'estompe et finit par se perdre dans les marais du bon goût et du beau linge.

\section{Le probable}

Le probable est l'ensemble des régularités de la nature. Dans les termes de la physique classique c'est l'entropie et le désordre croissant dans l'univers. Le probable est l'objet exclusif de la science : il n'y a de science que du probable et là où il n'y a pas de probable il ne peut pas y avoir de science. Le probable n'existe pas d'avance, évidemment, c'est la science qui le fabrique dans ses officines et en met partout où elle peut; quand elle ne réussit pas elle continue d'essayer. Tout scientifique est un entrepreneur en probable, quoiqu'il fasse et tout le long du jour il consomme, échange, produit et recycle du probable.

Gilles Deleuze, Nietzsche et la philosophie, p. 120. 
En logique le probable c'est trois choses : 1) l'opération réversible non orientée dans le temps ; 2) par conséquent c'est aussi l'éternité du concept (est éternel ce qui n'a pas de commencement et qui est hors-temps) ; 3) la puissance de la logique et qui lui vient de sa tautologie. La logique n'est pas une science mais seulement la gardienne du temple de l'éternité du concept, c'est-à-dire la gardienne de la principale fiction de la science en Occident.

Quand la question à l'ordre du jour est la construction d'un pont, d'un avion, d'un vaccin antivariolique ou l'une quelconque de ces merveilleuses rallonges que la science procure à l'autonomie de la personne, alors je préfère nettement l'intervention du scientifique à celle de l'artiste, c'est plus prudent. De même encore pour toute chose que l'on peut savoir, comme la vitesse de la lumière ou l'immunité relative contre le suicide conférée par tel ou tel facteur, le recours au probable est souhaitable.

Mais ce domaine du probable pourrait être élargi et transformé par la création d'une science de la morale interprétant les valeurs comme autant de contraintes. Car pendant que le corpus de connaissances scientifiques continuerait à s'accroître de la façon habituelle par l'accumulation de données nouvelles, l'entrée en scène d'une science de la morale changerait profondément quelques-unes des données en cause. Jusqu'ici, on l'a vu, le progrès de la science en matière morale se faisait toujours aux dépens du discours philosophique-théologique dans une sorte de «joute à somme nulle » où la première gagnait tout ce que l'autre perdait, cependant que la nature du processus scientifique demeurait essentiellement inchangée ; on se rappellera que Durkheim considérait les faits sociaux « comme des choses ", ce qui renforçait d'autant la prétention positiviste à produire un savoir libéré de toute considération de la valeur. Mais ce qui est maintenant en cause n'est plus seulement l'application des méthodes des sciences « dures » et de la statistique aux affaires humaines, c'est l'identification nouvelle des facteurs de moralité et d'immoralité en tant qu'ils constituent des régularités de la nature et de la nature humaine, ce qui est bien différent. Le problème se transforme dès le moment où une recherche expérimentale porte directement sur l'immoralité ellemême et parvient à en identifier les éléments et leur origine, lui donnant ainsi un fondement factuel qui la rend prévisible et donc manipulable. La création d'une telle science pourrait entraîner des conséquences non négligeables sur la démarche scientifique classique fondée sur la distinction canonique entre les faits et les valeurs.

Une première série de telles conséquences pourrait affecter le statut théorique de la science en son sens le plus élémentaire. On reconnaît depuis plusieurs années que la distinction entre les faits et les valeurs, sur laquelle la production des connaissances scientifiques reposait exclusivement jusqu'ici, s'est effritée parce que les faits eux-mêmes présupposent des valeurs ${ }^{1}$. Même lorsque l'incertitude

Hillary Putnam, Reason, Truth and History, p. 128. 
née de cette tendance s'accrut considérablement dans un champ scientifique particulier avec le théorème d'Heisenberg (j'y reviens à l'instant) cette promotion n'apporta pas de modification appréciable à la mesure de l'énormité de l'événement ni au statut épistémologique de ce que c'est qu'un fait et que le discours scientifique continue à considérer comme le construit value-free d'un pur observateur. Cette position était compréhensible tant que les valeurs morales se voyaient effectivement refuser tout accès au discours scientifique sous quelque forme que ce soit. Que se passerait-il si de telles valeurs faisaient leur entrée en science par l'identification de facteurs objectifs du comportement moral accessibles à l'expérimentation? Nul ne peut le dire. D'un point de vue philosophique un tel développement pourrait servir à accréditer davantage des thèses constructivistes comme celle de Nicholas Rescher ${ }^{1}$ qui met en relief l'influence de l'esprit humain dans la construction de ce que la science appelle des faits et que le commun des mortels finit, souvent avec plusieurs années de retard, par appeler la réalité. Les lois scientifiques, dit Rescher, ne peuvent éviter cet asservissement à l'esprit humain « qui les fait habiter cette région que [David] Hume voulait interdire à la pensée rationnelle : entre les questions de fait, d'une part, et les nécessités logiques d'autre part ${ }^{2}$ ». Selon ce point de vue les lois du probable ne sont pas découvertes, elles sont au contraire très littéralement construites, ce qui leur confère un caractère nettement anthropomorphique.

Une deuxième série de conséquences affecterait l'activité du scientifique en tant que praticien et maître d'œuvre en matière de probable qui aurait alors à rendre compte de l'introduction de facteurs de valeurs, sous forme de probable, dans le corpus de la science. Dans l'histoire de la science une telle invasion est inattendue et tout à fait indésirable. Avec le temps, à mesure que la position épistémologique du scientifique comme observateur pur devient de moins en moins tenable, celle de participant devient plus plausible et se renforce. Ce qui pourrait engendrer des conséquences inattendues puisque toute donnée scientifique est réputée théoriquement accessible, avec les précautions méthodologiques et les protocoles habituels, à toute autre entreprise scientifique quelle que soit sa nature ou ses objectifs. Au cours de la dernière décennie la communauté scientifique a montré des signes d'intérêt pour certaines questions morales particulières comme les droits de la personne, l'avortement, la protection de l'environnement et plusieurs autres domaines encore, mais la plupart de ces initiatives ont para motivées moins par un souci du progrès de la science elle-même que par les intérêts corporatistes de la communauté scientifique ou les convictions morales des scientifiques individuels. Tout comme un biologiste peut aussi détester la chasse à l'ours. À mesure que les facteurs des valeurs transformés en probable deviennent des faits incorporés à la connaissance scientifique, la pratique de la science pourrait s'enrichir d'un nouvel arsenal de moyens de traiter de nouveaux sujets, problèmes et visions scientifiques

Nicholas Rescher, Conceptual Idealism.

Op. cit., p. 74. 
aussi bien que moraux ou politiques. Cette pratique pourrait aussi ouvrir la porte à des discussions stériles et à des impasses. Probablement les deux.

Pour l'improbable c'est différent. L'improbable est plus proche du romantisme et de Goethe écrivant : « Tout ce que je sais n'importe qui peut le savoir, mais mon cœur est à moi. » L'improbable appartient aux choses que l'on fait sans en avoir de connaissance théorique ou exactement communicable par un discours articulé, comme écrire un poème, aller à cheval, réussir un gâteau, faire l'amour, plonger dans le sommeil. L'improbable habite la face nocturne de ce que nous savons faire sans savoir dire comment il faut s'y prendre, il fournit la recette du bonheur, l'écart inespéré du hasard sans avenir qui nous fait échapper pour une fois aux contraintes imposées par les trois idoles que la science nous a si bien appris à vénérer: la norme, la vérité, le groupe. C'est pourquoi l'exposé qui suit sur l'improbable prendra la forme inverse de trois éloges inspirés par la philosophie de Nietzsche : éloge de la négligence, éloge de la non-vérité, éloge de la trahison.

\section{Éloge de la négligence}

À première vue la négligence est un manque de soin, d'application ou d'exactitude, bref un laisser-aller. Pourtant le bon usage de la négligence est un élément-clé de toute démarche humaine, scientifique aussi bien que morale.

La connaissance scientifique du probable implique toujours deux choses. En premier lieu une évaluation qui est l'application d'une norme assurant un certain degré de précision ou d'exactitude dans l'application de la mesure, en deçà de laquelle la différence sera considérée d'importance négligeable. C'est ainsi qu'on trouve en science appliquée les concepts d'erreur standard et de déviation standard. Par exemple, dans une analyse statistique de génétique des populations, on convient ordinairement que $\mathrm{P}<0,05$ est la limite supérieure pour qu'une déviation soit considérée significative, c'est-à-dire suffisante pour n'être pas le fruit du hasard ; autrement elle sera considérée comme d'importance négligeable. Ce degré de précision relève d'une décision et celle-ci comporte nécessairement une part d'arbitraire. Dans le cas de la déviation qui n'est pas le fruit du hasard, puisqu'il n'y a pas de définition abstraite du hasard (c'est-à-dire non empirique) ${ }^{1}$, en dernière analyse cet arbitraire reposera sur l'empirisme du lancer des dés. La première condition de la mesure est la création arbitraire d'une norme.

En second lieu cette création de la norme requiert l'intervention du groupe. C'est le groupe, dans le cas de la déviation il s'agit du groupe des mathématiciens et des scientifiques, qui doit décider du degré de précision en deçà duquel, dira-t-il,

1 Ivar Ekeland, Le Calcul, l'Imprévu. Les Figures du temps de Kepler à Thom, p. 64-65 : « Il n'y a pas, je pense, de définition abstraite du hasard : aucune en tout cas qui soit cohérente. On y supplée par des définitions concrètes, qui en dernière analyse renvoient à l'expérience du lancer des dés. » 
la différence est d'importance négligeable. On voit que la négligence est une limite, inhérente mais seulement convenue et non théorisée, à l'action et à la connaissance. C'est le tissu conjonctif entre le savoir théorique individuel et le travail pratique du groupe.

Ainsi, par elle-même la négligence n'est certainement pas une faute, c'est plutôt un problème à gérer en permanence. Pourquoi donc nous a-t-on toujours présenté la négligence comme une faute ? Ce n'est là que le langage codé à l'usage du troupeau, la vraie faute est la négligence que le scientisme du groupe juge excessive ou au contraire insuffisante, intempestive, inopportune, mal à propos, inconvenante, de mauvais goût ou n'importe quoi. Il y a donc bonne négligence et mauvaise négligence. Qu'est-ce qu'une bonne négligence ?

Ça dépend. Pour les savants la bonne négligence c'est la science, pour les amants c'est l'improbable rendu facile, pour Arthur Rimbaud c'était être dans la nature comme avec une femme. La science est une fiction qui a de nombreux adeptes, la superstition est une autre fiction qui a aussi de nombreux adeptes. Les temps et les lieux propices sont différents et la bonne négligence varie.

Il en va de la bonne négligence comme du bon goût : ça ne se discute pas, ça s'éduque. Le bon goût est le goût particulier du groupe qui a le pouvoir de dire que son goût c'est le bon goût ; le bon goût est le goût plus le pouvoir, c'est ce qui le rend si follement désirable aux snobs. La bonne négligence est de même une négligence inthéorisée pourvue du pouvoir du groupe scientifique qui l'impose.

Le même raisonnement s'applique à toute mesure. Nous commençons par reconnaître que nous ne mesurons pas le temps, toute mesure du temps ne mesure en fait que l'espace, comme le déplacement des aiguilles sur la face de l'horloge. Puis nous comprenons que nous ne mesurons même pas l'espace mais seulement la mesure elle-même. Dire qu'un objet mesure un mètre signifie que l'étalon a été appliqué selon les règles convenues et les tolérances désirées: avec bonne négligence. Ce n'est qu'à partir de ce point que la formation scientifique peut commencer.

La bonne négligence est celle que nous pratiquons ensemble mais sans nécessairement pouvoir la dire. Par exemple, nous savons depuis 1927 et Heisenberg qu'il n'y a pas d'observateur pur qui ne soit en même temps un participant, puisque le système observé est lie en permanence au système observant et qu'il y a donc des limites théoriques absolues à la précision des observations et des calculs. Pourtant, précisément à la même époque, c'est la revendication du statut d'observateur le plus pur jamais imaginé qui a fait la fortune du positivisme, de l'empirisme logique et du Cercle de Vienne dans les années 1930, prometteuse de cinquante ans de stupidité positiviste. 
Pourtant la pratique du positivisme n'est possible que si elle demeure modérée, entravée et en partie désavouée. Le positiviste ne peut demeurer logique que s'il apprend à reconnaître d'une manière ou d'une autre les circonstances où il convient de l'être moins ou de cesser entièrement de l'être, autrement dit les limites pratiques (non théorisées) de l'usage de la logique. Ce qui requiert un apprentissage dans le bon usage de la négligence, qui est l'inconséquence que l'ensemble des positivistes pratiqueront, mais ensemble.

La bonne négligence ressemble aussi au bon goût en ce qu'on ne peut pas l'apprendre formellement dans des livres mais seulement de manière informelle dans des groupes (composés de personnes qui définissent la bonne négligence et qui siègent sur des comités chargés de la distribution des bourses d'étude et des fonds de recherche), ce qui la met à l'abri de l'influence de personnes extérieures au groupe; le double standard de Garrett Hardin à son meilleur. La bonne négligence fournit le ciment négatif nécessaire à la cohésion du groupe : la bonne négligence est celle que l'on partage et rien d'autre. Les membres du groupe sont unis entre eux par leurs inconséquences partagées.

On chercherait en vain des livres définissant la bonne négligence, et même si de tels livres existaient ils seraient tout aussi inutiles que des ouvrages qui tenteraient de définir le bon goût. Le problème fondamental n'est pas que toute mesure contient une part d'arbitraire, c'est que le positivisme ne veut pas entendre parler d'un système dans lequel la précision de la norme est assurée en pratique par une forme particulière de bonne négligence imposée par un groupe historique. Le problème est la dénégation de l'influence du groupe.

Comme on voit la bonne négligence n'est pas une petite affaire, c'est un domaine qui demande une grande attention mais qui n'est évidemment pas limité à la science et n'est pas le monopole d'un groupe plutôt que d'un autre. Cela nous concerne tous, nous sommes tous dans ce bain, nous sommes tous des vivants, et tout artiste ou créateur peut considérer à l'occasion que la science elle-même est d'importance négligeable et appliquer la règle de la bonne négligence à la vérité.

\section{Éloge de la non-vérité}

La volonté de René Descartes d'imprimer à la philosophie la clarté et la distinction de la géométrie lui inspira cette définition de la vérité : est vrai tout ce qui est clair et distinct. Marshall McLuhan ${ }^{1}$ commentant ce qu'il a appelé cette inopportune obsession cartésienne pour la clarté et la distinction qui impose à la philosophie la précision de la mathématique, affirme qu'elle n'aura finalement servi qu'à expulser de la philosophie la plupart des questions d'intérêt philosophique. C'est ce qui est en question ici.

Marshall McLuhan, Understanding Media : The Extensions of Man, p. 148. 
Qu'y a-t-il d'autre que la vérité, demandera-t-on? Il y a le théâtre de Shakespeare pour ceux que ses personnages intéressent et dont la vérité historique est sans importance. Il y a aussi Charles Baudelaire, Arthur Rimbaud, Salman Rushdie, la Bible et le Coran. Il y a l'histoire que les historiens réécrivent sans cesse, le théâtre de Samuel Beckett, François Rabelais et Carlos Castaneda. Bref, c'est l'ensemble de la production culturelle et artistique que l'on mésestime quand on n'a d'yeux que pour la vérité.

L'éloge de la non-vérité n'est donc pas l'éloge du mensonge ni du fauxsemblant ou de l'erreur. Le problème n'est pas là. C'est alors que les choses dont la vérité témoigne sont dans le temps, qu'elle-même revêt spontanément un uniforme qui la présente comme si elle était hors-temps. La vérité n'aime rien mieux que l'uniforme, en fait la vérité aime sans merci tous les uniformes et éprouve la plus grande répugnance pour tout ce qui manque de forme et pour le multiforme. Avec le résultat que ce qui s'était passé dans le temps devient méconnaissable a nos yeux et que nous-mêmes avons alors grand-peine à exister. Si Dieu n'existe pas tout est possible mais la vérité éternelle est sans remède. Chaque fois que la vérité s'exhibe revêtue de son uniforme hors-temps elle fait de nous des errants incapables d'avoir lieu. Seul le temps arrive aux hommes.

Selon Tzvetan Todorov ${ }^{1}$, Nietzsche fut le premier à dire qu'il n'y a pas de faits mais seulement des interprétations, et cette opinion fut répétée par la suite par de nombreux auteurs et de multiples façons. «La formule complète, dit Todorov, serait du reste : il n'y a pas de faits, mais seulement des discours sur les faits ; par conséquent, il n'y a pas de vérité du monde, mais seulement des interprétations du monde. »

Ce qui soulève le problème de la nature de la vérité. La vérité, dit Todorov, peut prendre deux formes : 1) la vérité-adéquation qui concerne le oui et le non, le tout ou rien, par exemple lorsque le juge veut savoir si l'accusé a ou non accompli une action ; 2) le second sens est la vérité-dévoilement graduel de quelque chose : croyance, sens ou drame. Ce second sens de la vérité ne résulte pas de l'alternative d'un tout ou rien, c'est plutôt une question de plus ou de moins. Et ce second sens est dit supérieur au premier. La question se pose alors de savoir comment passer du premier au second sens et en quoi la vérité-dévoilement est supérieure à la vérité-adéquation. Il y faut une prise de position morale, dit Todorov :

Si la vérité est soumise à la morale, s'il n'existe de vérités que pragmatiques, qui prendra la décision de ce qui est plus vrai et plus philosophique que la vérité ? Le philosophe-roi ? La majorité des citoyens? Ces solutions présentent quelques inconvénients bien connus, qu'on a tendance à oublier parfois. Mais si on évite la

Zvetan Todorov, Les Morales de l'histoire, p. 130 et sv. 
subordination d'un type de vérité à un autre, ou même toute continuité, comment situer les deux dans un cadre unique ${ }^{1}$ ?

Posée en termes différents la question devient : est-il possible de proposer un seul cadre conceptuel qui engloberait adéquatement les deux sens de la vérité ? Todorov ne propose pas de réponse à sa question. Pour notre part, nous savons que c'est l'affaire du groupe que d'imposer des contraintes secondaires. La vérité selon le premier sens se prête à toute vérification empirique faite par n'importe qui alors que le second sens est différent en ce qu'il implique les valeurs elles-mêmes, sa supériorité alléguée signifie que seul un membre du groupe est normalement en mesure d'attester sa présence et son contenu. Dans Hamlet, par exemple, il y a une vérité-dévoilement dont on peut attester mais qu'on ne peut certes pas démontrer.

La recherche de la vérité est souvent un mode de dénégation du passage du temps. Par exemple, on dira qu'il y a conformité entre A et B. Si A est situé dans le présent $\mathrm{B}$ est probablement dans le passé. Dans ce genre de comparaison, $\mathrm{B}$, quel qu'il soit, sert de norme et toute norme est la fiction convenue du groupe qui la crée et qui lui confère son autorité, j'entends l'autorité qu'il possède comme groupe. La vérité est le premier sacrement du groupe, la recherche de la non-vérité rend possible le premier pas vers l'individuation. La passion pour la vérité résulte d'un violent besoin de retrouver l'odeur du troupeau. Le premier rôle de l'intemporalité de l'uniforme logique dont le groupe revêt sa vérité est de faire croire à son éternité, à cela qui n'est jamais vrai, toutes les vérités ayant une date de naissance, et c'est essentiellement ce que nous a enseigné Nietzsche, toutes les vérités sont éventuellement jetables et avec elles les groupes qui en vivent.

La non-vérité est la façon typiquement humaine d'être un animal. En Occident la façon typiquement humaine d'être un animal est de faire semblant de ne pas l'être ou de l'être autrement, pensons-nous. Tout est là. La non-vérité c'est l'unité que notre vie morale fracture et ressoude dans le grand concert qui rétablit l'harmonie entre nous et tous les autres animaux et le ciel et la terre et nos ancêtres et nos enfants les dieux. Quelle famille ! Nietzsche dit que nous sommes une terre arable pour les choses. Nous les aveugles voyons que le temps c'est nous.

La fortune de Michel Foucault comme philosophe et historien français aura été de nous enseigner ce que c'est que l'humanisme. Sa démonstration nous en a montré le lieu et la date de naissance. Mais dès que nous eûmes ainsi appris que l'humanisme est né un jour et donc qu'il n'est pas de tout temps, beaucoup de gens se sont empressés de proclamer la fin de l'humanisme et d'évoquer ce qui a été appelé « la mort de l'homme » ou du modèle que l'humanisme inspirait. Ce n'était que jactance, car l'humanisme n'a rien perdu de son importance après la démonstration de Foucault, rien ne l'a remplacé dans le discours moral qui, pour le moment du moins, ne saurait se priver d'une référence à ce qu'est l'être humain et à

Op. cit., p. 133. 
ce que nous voulons qu'il devienne. Mais Foucault a tout de même pratiqué une petite brèche dans la cohésion du groupe.

Dernier exemple, dans l'œuvre de Carlos Castaneda, la question de la vérité factuelle ou historique de son personnage central Don Juan : ce sorcier Yaqui a-t-il vraiment existé et l'anthropologue l'a-t-il vraiment rencontré comme il le prétend dans une ville du Mexique, ou n'est-ce là que l'invention d'un auteur à l'imagination fertile nommé Carlos Castaneda ? Les gens qui posent ce genre de questions sur la " vérité » du personnage laissent entendre que la valeur de l'œuvre serait grandement diminuée s'il se trouvait que le sorcier Don Juan n'a jamais existé. Je soutiendrais au contraire qu'en ce cas notre admiration pour cette œuvre ne pourrait que s'en trouver accrue. Car le couple maître-disciple formé par Don Juan et Carlos est un moyen d'expression incomparablement plus riche d'enseignements que la simple question de l'existence historique des protagonistes. L'un des plus illustres de ces couples maître disciple est celui de Socrate et Platon. Si nous supposons un instant que Socrate n'a jamais existé et que son personnage n'est qu'un simple procédé littéraire mis en œuvre par le philosophe Platon, notre admiration pour son œuvre s'en trouvera cent fois accrue et non pas diminuée.

\section{Éloge de la trahison}

La grégarité de l'être humain en fait un veau, un veau qui serait parlant. Le probable prend la forme d'une fidélité à la norme édictée par le groupe pour chacune des conduites décrites par Levine et Campbel ${ }^{1}$ qui, comme on l'a vu dans l'introduction, ont défini le syndrome de l'ethnocentrisme dans les termes suivants : «Se voir soi-même et son groupe comme vertueux et supérieurs, voir les gens extérieurs au groupe comme méprisables, immoraux et inférieurs. »

L'une des façons d'accéder à l'improbable est la trahison qui consiste à montrer plus de fidélité à soi-même et à son propre sens du devoir qu'à celui du groupe. C'est ce que montrèrent chacun à sa façon Roméo et Juliette dans le no mans land séparant leurs familles ennemies, Charles Baudelaire des Fleurs du mal qui invente une nouvelle sensibilité poétique. En politique ce fut la voie choisie par Thomas Jefferson et George Washington en 1776, ou, plus près de nous, par Charles de Gaulle et son appel à la trahison en 1940. N'importe qui peut trahir, il suffit qu'une personne choisisse de parler et d'agir de telle sorte que son individuation devienne manifeste et tôt ou tard ses collègues et elle-même l'accuseront de trahison. Choisir, c'est trahir. L'improbable est la solitude préalable et conséquente à cette trahison.

J'ai montré plus haut que la morale d'un groupe est l'ensemble de ses contraintes secondaires qui forment ses valeurs. Trahir, c'est abandonner cette

\footnotetext{
R. A. LeVine et D.T. Campbell, Ethnocentrism: Theories of Conflict, Ethnic Attitudes and
} Group Behavior, p. 12. 
contrainte secondaire reconnue, c'est manquer à cette fidélité (ou être perçu comme manquant, le résultat est le même) à la morale du groupe. La trahison est un manque à une fidélité reconnue en faveur d'une autre qui ne l'est pas encore et que le groupe n'honorera que lorsqu'il aura choisi d'en faire sa nouvelle valeur, ce qui dépend souvent des aléas de l'histoire. En 1940, Charles de Gaulle se montra capable de violer sa parole de soldat mais c'est le " sauveur » que sa nation honore depuis ce temps et non pas le traître. Cette différence tient en bonne partie au sort des armes; un autre dénouement de la guerre 1939-1945 lui aurait valu d'être appelé " officier félon », " arriviste », « fourbe », " imposteur ». La langue française est très riche pour dire la trahison.

Revenons en terminant sur le piège à singe des Indiens dont parle Robert $\mathrm{M}$. Pirsig. Comme tout mode de communication, notre savoir scientifique est une alliance qui nous lie au groupe de toute la puissance qu'il lui confère. La sagesse serait de savoir parfois ouvrir la main, renonçant ainsi à une partie des avantages personnels qui nous viennent d'appartenir à ce pouvoir : le singe s'enfuirait alors dans la périlleuse autonomie retrouvée par la trahison que le groupe ne manquerait pas de voir dans son geste. C'est quand on consent à être illogique que l'on commence à être personnel, mais qui osera conseiller l'illogisme a un Occidental avec l'espérance d'être entendu ? Il semblerait que rien ne serait plus précieux que la possession d'une idée-maîtresse autorisant d'avance une semblable trahison, en vérité ce serait l'équivalent d'une recette de la sagesse. Car la lecture de saint Augustin nous persuade que c'est la notion de péché, dans l'ancienne métaphysique de la tradition judéo-chrétienne, qui a pratiqué la première fracture dans l'harmonie insécable de l'« Être » en Occident. C'est cette fissure dans l'être, cette felix culpa que quelques hommes de l'époque disaient scandaleuse et beaucoup d'autres insignifiante qui rendit possible, mais beaucoup plus tard, le Je de Descartes et le Sujet de Kant. C'est pourquoi en définitive, dans l'histoire, la démocratie est demeurée si intimement liée à la culture chrétienne de la morale personnelle et à la science. Pour notre part nous aurions évidemment intérêt à connaître la recette du bon usage de la trahison, si une telle recette existait, mais il n'y en a pas. Pour trahir il faut avoir deux fidélités et donc une de trop et donc être seul.

N'importe qui peut être illogique (ou logique) mais jamais à lui tout seul, il faut s'y mettre à plusieurs et l'être ensemble, telle est la définition du groupe et qui aide à comprendre la grande importance que le groupe accorde au double standard de moralité frappant quiconque veut y entrer ou en sortir.

La trahison est toujours immorale, au sens donné au chapitre 2 où l'on voit que l'immoralité fait partie de la morale. On ne saurait en parler très longuement car même le simple éloge de la trahison semble immoral, ce qui montre combien grande est l'emprise du groupe. Parmi les nombreux lapidés de l'histoire du peuple juif un certain nombre furent appelés prophètes, généralement après leur mort. La trahison attriste en annonçant l'improbable et cela aussi appartient à la philosophie. 


\section{BILIOGRAPHIE}

$\underline{\text { Retour à la table des matières }}$

Apostle, Richard A., Glock, Charles Y, Piazza, Thomas, Svelze, Marijean (1983).

The Anatomy of Racial Attitudes, Berkeley, University of California Press.

Aristote (1985). Éthique de Nicomaque, texte, traduction, préface et notes par Jean Voilquin, Paris, Garnier.

Bateson, Gregory (1977). Vers une écologie de l'esprit, 2 vol., Paris, Seuil.

Benedict, Ruth (1946). The Chrysantemum and the Sword : Patterns of Japanese Culture, Boston, Houghton Mifflin.

Bernard, Claude (1966). Leçons sur les phénomènes de la vie communs aux animaux et aux végétaux, préface de Georges Canguilhem, Paris, Librairie Philosophique Vrin.

Brown, William L. Jr. (1987). « Punctuated Equilibrium Excused : The Original Examples Fail to Support It », Biological Journal of the Linnean Society, 31, p. 383-404.

Camus, Albert (1942). Le Mythe de Sisyphe, Paris, Gallimard.

Castaneda, Carlos (1988). Le Voyage à Ixtlan. Les Leçons de Don Juan, trad. Marcel Kahn, Paris, Gallimard.

Collins, Anne (1990). Les Patients du docteur Cameron : L'étonnante histoire des expériences de lavage de cerveaux financées par la CIA dans un hôpital canadien, Montréal, Éditions de l'Homme.

Comford, Francis Macdonald (1957), From Religion to, Philosophy. A Study in the Origins of Western Speculation, New York, Harper \& Row.

Darwin, Charles (1973). L'Origine des espèces au moyen de la sélection naturelle. $\mathrm{Ou}$ la lutte pour l'existence dans la nature, Verviers (Belgique), Éditions Gérard \& Co. [Texte disponible dans Les Classiques des sciences sociales. JMT.]

Dawkins, Richard (1978). Le Gène égoïste, trad. Julie Pavesi et Nadine Chaptal, Paris, Éditions Menges.

Dawkins, Richard (1989). L'Horloger aveugle, trad. Bernard Sigaud, Paris, Robert Laffont.

Deleuze, Gilles (1962). Nietzsche et la philosophie, Presses Universitaires de France, Paris.

Dobzhansky, Theodosius (1971). « Race Equality », in Richard H. Osborne (sous la direction de), The Biological and Social Meaning of Race, San Francisco, W.H. Freeman and Company.

Dodds, E. R. (1965). Les Grecs et l'irrationnel, Paris, Aubier. 
Dovidio, John F., Gaertner, Samuel L. (1986). «Prejudice, Discrimination, and Racism : Historical Trends and Contemporary Approaches », in Dovidio, John F., Gaertner, Samuel L. (sous la direction de), Prejudice, Discrimination, and Racism, Orlando Florida, Academic Press.

Durkheim, Émile (1967). Le Suicide. Étude de sociologie, 2e éd., Paris, Presses Universitaires de France. [Texte disponible dans Les Classiques des sciences sociales. JMT.]

Ekeland, Ivar (1984). Le Calcul, l'Imprévu. Les Figures du temps de Kepler à Thom, Paris, Seuil.

Flacelière, Robert (1959), La Vie quotidienne en Grèce au siècle de Périclès, Paris, Hachette.

Forsé, Michel (1989). L'Ordre improbable, Paris, Presses Universitaires de France.

Foucault, Michel (1966). Les Mots et les Choses. Une archéologie des sciences humaines, Paris, Gallimard.

Gould, Stephen Jay (1989). Wonderful Life. The Burgess Shale and the Nature of History, New York, Norton.

Gould, S. J. et Lewontin, R., " The Spandrels of San Marco and the Panglossian Paradigm : A Critique of the Adaptationist Programme ». Proceedings of the Royal Society of London, 205, p. 581-598.

Gould, S. J. et Eldredge, N. (1977), « Punctuated Equilibrium : the Tempo and Mode of Evolution Reconsidered », Paleobiology, 3, p. 115-151.

Hamilton, William D. (1964). « The Genetic Evolution of Social Behaviour », I et II, Journal of Theoretical Biology, vol. 7.

Hardin, Garrett (1972). "Population Skeletons in the Environmental Closet», Bulletin of the Atomic Scientists, juin, p. 37-41.

Havelock, Eric A. (1963), Preface to Plato, Cambridge, Mass., Harvard University Press.

Hillman, James (1989). A Blue Fire : Selected Writings by James Hillman, New York, Harper \& Row.

Hobbes, Thomas (1960). Leviathan; or the Matter, Forme, \& Power of a Commonwealth Ecclesiastical and Civil, Oxford, Blackwell. [Texte disponible en version française dans Les Classiques des sciences sociales. JMT.]

Hosken, Fran (1982). The Hosken Report: Genital and Sexual Mutilation of Females, 3e éd. rev., Lexington, Mass., Women's International Network News.

Hulse, Frederick S., "Social Behavior and Human Diversity », in Richard H. Osborne (1971) (sous la direction de), The Biological and Social Meaning of Race, San Francisco, W. H. Freeman and Company.

Hume, David (1958). Treatise of Human Nature, Being an Attempt to Introduce the Experimental Method of Reasoning into Moral Subjects, Oxford, Clarendon Press. [Texte disponible dans Les Classiques des sciences sociales. JMT.] 
Ike, Ben W. (1987), « Man's Limited Sympathy as a Consequence of his Evolution in Small Kin Groups », in Vernon Reynolds, Vincent Falger et Ian Vine (sous la direction de), The Sociobiology of Ethnocentrism : Evolutionary Dimensions of Xenophobia, Discrimination, Racism and Nationalism, Londres, Croom Helm, p. 216-234.

Illich, Ivan (1975). Némésis médicale. L'Expropriation de la santé, Paris, Seuil.

Kant, Immanuel (1950). Critique de la raison pure, trad. A. Tremesaygues et B. Pacaud, Paris, Presses Universitaires de France.

Korzybski, Alfred (1949). Science and Sanity. An Introduction to Non-Aristotelian Systems and General Semantics, 3e éd., Lakeville, Conn., The International Non-Aristotelian Library Publishing Company.

Lagadec, Claude (1982). Dominances. Essai de sociobiologie sur l'inégalité et la tromperie, Longueuil, Les Éditions du Préambule. [Texte disponible dans Les Classiques des sciences sociales. JMT.]

Lagadec, Claude (1984). La Morale de la liberté, Longueuil, Les Éditions du Préambule. [Texte disponible dans Les Classiques des sciences sociales. JMT.]

Lagadec, Claude (1992). «Un point de vue moral : Le probable et l'improbable », in Roger Tessier et Yvan Tellier (sous la direction de), Changement planifié et développement des organisations, Presses de l'Université du Québec, Québec, volume 8, p. 513-527. [Texte disponible dans Les Classiques des sciences sociales. JMT.]

Lévi-Strauss, Claude (1967). Les Structures élémentaires de la parenté, 2e éd., Paris-La Haye, Mouton \& Co.

LeVine, R.A. Campbell, D.T. (1972). Ethnocentrism : Theories of Conflict, Ethnic Attitudes and Group Behavior, New York, Wiley.

Lewin, Kurt (1945). «Conduite, connaissance et acceptation de nouvelles valeurs ", traduction Claude Lagadec, in Roger Tessier et Yvan Tellier, Changement planifié et développement des organisations, Presses de l'Université du Québec, 1991, vol. 6, p. 1-12.

Lichtenhaeler, Charles (1978). Histoire de la médecine, Paris, Fayard.

Lumsden, Charles J., et Wilson, Edward O. (1981). Genes, Mind and Culture, Harvard University Press, Cambridge, Massachusetts.

Lumsden, Charles J., et Wilson, Edward O. (1983). Promethean Fire. Reflections on the Origin of Mind, Harvard University Press, Cambridge, Massachusetts.

Mabbott, John David (1959). Article « Free Will », Encyclopœdia Britannica.

Maynard Smith, J. (1978). The Evolution of Sex, London, Cambridge University Press.

Mayr, Ernst (1991). One Long Argument. Charles Darwin and the Genesis of Modern Evolutionary Thought, Cambridge, Mass, Harvard University Press.

McLuhan, Marshall (1964). Understanding Media: The Extensions of Man, Toronto, Signet Books. 
Minear, Richard H. (1971) Victors' justice; the Tokyo War Crimes Trial, Princeton, Princeton University Press.

Moore, G.E. (1968). Principia Ethica, London, Cambridge University Press.

Nietzsche, Friedrich (1976). Euvres philosophiques complètes, texte établi par G. Colli et M. Molinari, trad. Pierre Klossowski, Paris, Gallimard, tome XIII.

Pirsig, Robert, M. (1974). Traité du zen et de l'entretien des motocyclettes, trad. Maurice Pons, Andrée et Sophie Mayoux, Paris, Seuil.

Platon, Euvres complètes (1959), tome VI, La République, texte établi et traduit par Émile Chambry, Paris, Les Belles Lettres.

Putnam, Hillary (1981). Reason, Truth and History, Cambridge, Cambridge University Press.

Rawls, John (1971). A Theory of Justice, Cambridge, Mass., Harvard University Press.

Rescher, Nicholas (1973). Conceptual Idealism, Oxford, Basil Blackwell.

Rescher, Nicholas (1984). The Limits of Science, Berkeley, University of California Press.

Reynolds, V. (1980), The Biology of Human Action, 2 éd., Oxford, Freeman.

Rousseau, Jean-Jacques (1837). Euvres complètes, 4 tomes, Paris, A. Desrez, Libraire-éditeur.

Ruse, Michael, 1986. Taking Darwin seriously. A naturalistic approach to Philosophy, Oxford, Basic Blackwell.

Russell, Bertrand (1945). History of Western Philosophy and its Connections with political and social circumstances, from the earliest times to the present day, New York, Simon \& Schuster.

Sartre, Jean-Paul (1943). L'Être et le Néant, Paris, Gallimard.

Southwick, C. H. (1967). «An Experimental Study of Intragroup Agonistic Behavior in Rhesus Monkeys (Macaca mulatta) », Behaviour, 28 (1, 2), 182209.

Southwick, C. H. (1969). «Aggressive Behaviour of Rhesus Monkeys in Natural and Captive Groups ». In S. Garattini and E. B. Sigg, (sous la direction de), Aggressive Behavior, Proceedings of the Symposium on the Biology of Aggressive Behaviour, Milan, May 1968. Excerpta Medica, Amsterdam, 32 : 43.

Steiner, George (1978). Après Babel. Une poétique du dire et de la traduction, Paris, Albin Michel.

Sumner, W.G. (1906). Folkways, New York, Ginn.

Tiger, Lionel (1969). Men in Groups, London, Nelson.

Todorov, Tzvetan (1991). Les Morales de l'histoire, Paris, Grasset.

Van der Dennen, Johan M.G. (1987), « Ethnocentrism and In-Group/ Out-Group Differentiation: A Review and Interpretation of the Literature », in Vernon Reynolds, Vincent Falger et Ian Vine (sous la direction de), The Sociobiology 
of Ethnocentrism : Evolutionary Dimensions of Xenophobia, Discrimination, Racism and Nationalism, Londres, Croom Helm, p. 1-47.

Vernant, Jean-Pierre, et Vidal-Naquet, Pierre (1981). Mythe et Tragédie dans la Grèce ancienne, Paris, Maspéro.

Wilson, Edward O. (1975). Sociobiology: The New Synthesis, Cambridge, Mass., Harvard University Press. 